\title{
Abordagem Semi-Paramétrica para Cópulas Variantes no Tempo em Séries Temporais Financeiras
}

Daniel de Brito Reis

\author{
DissertaÇÃo APRESENTADA \\ $\mathrm{AO}$ \\ Instituto de Matemática e Estatística \\ $\mathrm{DA}$ \\ Universidade de SÃo Paulo \\ PARA \\ OBTENÇÃO DO TÍTULO \\ $\mathrm{DE}$ \\ Mestre em CiênCIAS \\ Área de Concentração: Estatística \\ Orientadora: Prof ${ }^{a}$. Dra. Chang Chiann
}

Durante o desenvolvimento deste trabalho o autor recebeu auxílio financeiro da CAPES

São Paulo, Setembro de 2016 


\section{Abordagem Semi-Paramétrica para Cópulas Variantes no Tempo em Séries Temporais Financeiras}

Esta versão da dissertação contém as correções e alterações sugeridas pela Comissão Julgadora durante a defesa da versão original do trabalho, realizada em 21/09/2016. Uma cópia da versão original está disponível no

Instituto de Matemática e Estatística da Universidade de São Paulo.

Comissão Julgadora:

- Prof ${ }^{\mathrm{a}}$. Dr ${ }^{\mathrm{a}}$. Chang Chiann - IME-USP

- Prof ${ }^{\mathrm{a}}$. Dr ${ }^{\mathrm{a}}$. Clélia Toloi - IME-USP

- Prof ${ }^{\text {a }}$. Dr ${ }^{\text {a }}$. Thelma Sáfadi - UFLA 
Canção do Rei Sol

Ó Mestre, fazei que eu procure mais consolar que ser consolado, compreender que ser compreendido, amar que ser amado.

Pois é dando, que se recebe,

é perdoando que se é perdoado e é morrendo que se vive para a vida eterna!

Maria Maria

Maria, Maria, é um dom, uma certa magia, uma força que nos alerta. Uma mulher que merece viver e amar como outra qualquer do planeta Maria, Maria é o som, é a cor, é o suor, é a dose mais forte e lenta. De uma gente que ri quando deve chorar e não vive, apenas aguenta Mas é preciso ter força É preciso ter raça

É preciso ter gana sempre Quem traz no corpo a marca Maria, Maria Mistura a dor e a alegria. Mas é preciso ter manha É preciso ter graça É preciso ter sonho sempre Quem traz na pele essa marca Possui a estranha mania De ter fé na vida Obrigado Mulher Guerreira, Minha Mãe e Avó do Kevin! 


\section{Agradecimentos}

Minha mais profunda e eterna gratidão fica, primeiramente a Maria das Mercês Soares de Brito (Dna Mercês), mãe e mulher guerreira, com quem aprendi a continuar lutando sempre, a olhar e manter uma postura positiva frente aos desafios que a vida impõe. A ela, por todo apoio incondicional durante essa longa jornada, o meu muitíssimo obrigado. Mãe muitíssimo obrigado!!

Agradeço a Prof ${ }^{a}$ Chang pelo apoio, desde a carta de recomendação até a finalização deste trabalho. Horas e horas de reuniões estão aqui impressas. Muito obrigado Professora, por sua paciência e apoio.

Minha eterna e fiel gratidão aos fieis amigos: Gilberto Pereira Sassi (Tontom), Marcos Rafael Nogueira Cavalcante (Monsenhor) pelas ideias, dedicação e apoio incondicional. Sem esses, o trabalho que aqui se encontra, provavelmente teria menos substância e rigor matemático.

Aos não menos fieis amigos: Kaique Mathias de Andrade Roberto (Mum-Rá), Bruno Monte de Castro (Excelência), Bruno Santos, Eliardo Costa (Ninja dos Orixás), Antônio Marcos (Sem Acento). Sem esses, o trabalho que aqui se encontra, seria bem menos do que é.

Outros amigos importantes e colegas do IME também merecem serem lembrados: Ariadne, Jean, Azrielex, Mel, Nathália Demétrio, Júlio Trecenti, Felipe Bhering. Vocês me ensinaram muito mais do quê matemática. Me ensinaram o quão é importante é a vivência acadêmica!

Por fim, e ainda tão importante, aos meus irmãos Lucas, Zoca, Valéria, Everaldo e Jacson, meu filho Kevin Gomes e sua mãe Sylmara Kiss, obrigado pela paciência durante a realização deste trabalho. 
Numa realização de grande alcance, nenhum homem pode conseguir resultados duradouros sem o auxílio e a cooperação de outros. A evolução da humanidade só se dará através da cooperação em benefício de todos. É impossível chegar lá sozinho.

Por sua vez, a Meritocracia é a fantasia contada por aqueles que, em uma posição ou condição privilegiada, tentam justificar e ratificar os insucessos e anonimato dos outros, que não partiram de condições tão privilegiadas quanto as suas.

"Sem saber que era impossível, com a ajuda dos amigos, dos professores e apoio da família, ele foi lá e fez!" 


\section{Resumo}

REIS, Daniel de Brito. Abordagem Semi-Paramétrica para Cópulas Variantes no Tempo em Séries Temporais Financeiras. 2016. Dissertação - Instituto de Matemática e Estatística, Universidade de São Paulo, São Paulo, 2016.

Neste trabalho foram utilizadas cópulas bivariadas variantes no tempo para modelar a dependência entre séries de retornos financeiros. O objetivo deste trabalho é apresentar uma abordagem de estimação semi-paramétrica de cópulas variantes no tempo a partir de uma função de cópula paramétrica na qual o parâmetro varia no tempo. A função do parâmetro desconhecido será estimada pela aproximação de ondaleta Haar, polinômio de Taylor e Kernel. O desempenho dos três métodos de aproximação será comparado via estudos de simulação. Uma aplicação aos dados reais será apresentada para ilustrar a metodologia estudada.

Palavras-chave: Cópulas variantes no tempo; Séries temporais; Ondaletas Haar; Polinômios de Taylor; Kernel. 


\section{Abstract}

REIS, Daniel de Brito. Semiparametric approach for time-varying copula in finacial time series. 2016. Dissertação - Instituto de Matemática e Estatística, Universidade de São Paulo, São Paulo, 2016.

In this work the bivariate Time-varying copula models have been used to model the dependence between payback. The aim of this work is to present an approach of semiparametric estimation of Time-varying copula models from a parametric copula function in which the parameter varies with the time. The function of the unknown parameter will be estimated by Haar wavelet approach, Taylor series and smoothing Kernel approximation. The measured performance of the three estimation method will be compared by simulation study. An application of the data will be presented to illustrate the studied methodology.

Keywords: Time-varying copula models ; Time Series; Wavelets Haar; smoothing Kernel approximation; Taylor series. 


\section{Sumário}

Lista de Figuras $\quad$ xi

Lista de Tabelas $\quad$ xiii

$\begin{array}{lll}1 & \text { Introdução } & 1\end{array}$

2 Conceitos básicos $\quad 5$

$2.1 \quad$ Séries temporais $\ldots \ldots \ldots \ldots \ldots \ldots$. . . . . . . . . . . . . . . . . . 5

2.1 .1 Estacionariedade . . . . . . . . . . . . . . . . . 6

2.1 .2 Ruído branco . . . . . . . . . . . . . . . . . . . . . . 7

$2.1 .3 \quad$ Função de autocovariância e função de autocorrelação . . . . . . . . . . 7

2.2 Ondaletas . . . . . . . . . . . . . . . . . . . . . . . . . . . 9

2.2 .1 Função Escala e Função Ondaleta . . . . . . . . . . . . . . . . . . . . 9

2.3 Polinômio de Taylor . . . . . . . . . . . . . . . . . . . . . . . . . . . . . . 14

2.4 Métodos de suavização por Kernel . . . . . . . . . . . . . . . . . . . . . . . . 16

$\begin{array}{lll}3 & \text { Cópula } & 19\end{array}$

3.1 Definições e propriedades básicas de Cópulas . . . . . . . . . . . . . . . . . . 20

3.1.1 $\quad$ Propriedades das distribuições . . . . . . . . . . . . . . . . . . . . . . 20

3.1 .2 Cópulas e Teorema de Sklar . . . . . . . . . . . . . . . . . . . . . . . 21

3.1.3 Os Limites de Fréchet-Hoeffding para distribuições conjuntas . . . . . 23

3.1 .4 Medidas de dependência . . . . . . . . . . . . . . . . . 25

$3.2 \quad$ Famílias de cópulas bivariadas . . . . . . . . . . . . . . . . . . . . . . . . . . 27

$3.2 .1 \quad$ Cópula Normal . . . . . . . . . . . . . . . . . . . . . . . . . . . . . . 28

3.2.2 Cópula Clayton . . . . . . . . . . . . . . . . . . . . . . . . . . . 29

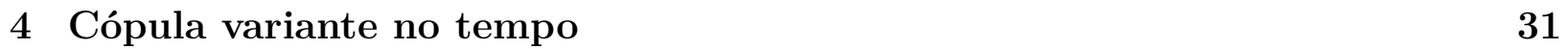

4.1 Motivação . . . . . . . . . . . . . . . . . . . . . . . . . . . . . . 31

4.2 Cópula variante no tempo $\ldots \ldots \ldots \ldots$ 
$4.2 .1 \quad$ Cópula Clayton variante no tempo . . . . . . . . . . . . . . . . . . . 39

4.2 .2 Cópula Normal variante no tempo . . . . . . . . . . . . . . . . . . . . 40

4.3 Estimação . . . . . . . . . . . . . . . . . . . . . . . . . . . . . . . . 41

\begin{tabular}{|lll}
\hline 5 & Estudo de simulações e aplicação & 43
\end{tabular}

5.1 Simulação . . . . . . . . . . . . . . . . . . . . . . . . . 43

5.2 Resultados do estudo de simulação . . . . . . . . . . . . . . . . . . . 48

$5.2 .1 \quad$ Caso Clayton . . . . . . . . . . . . . . . . . . . . . . . . . . . . . . 49

5.2 .2 Caso Normal . . . . . . . . . . . . . . . . . . . . . . . . 52

5.3 Aplicação . . . . . . . . . . . . . . . . . . . . 56

\begin{tabular}{|lll}
\hline 6 & Conclusão & 61
\end{tabular}

\begin{tabular}{|l|l|}
\hline A Código R & 63
\end{tabular}

A.1 Caso Clayton . . . . . . . . . . . . . . . . . . . 63

A.1.1 Constante . . . . . . . . . . . . . . . . . 63

A.1.2 Haar . . . . . . . . . . . . . . . . . . . . 66

A.1.3 Taylor . . . . . . . . . . . . . . . . . . . . 73

A.2 Normal. . . . . . . . . . . . . . . . . . . . . . . . . . . . 81

A.2.1 Constante . . . . . . . . . . . . . . . . . . . . . . 81

A.2.2 Haar . . . . . . . . . . . . . . . . . . . . 83

A.2.3 Taylor . . . . . . . . . . . . . . . . . . . . 88

A.2.4 Kernel . . . . . . . . . . . . . . . . . . . . . . . . . . . . . . . . . . . . . . . . 92

\begin{tabular}{lr}
\hline Referências Bibliográficas & 97
\end{tabular} 


\section{Lista de Figuras}

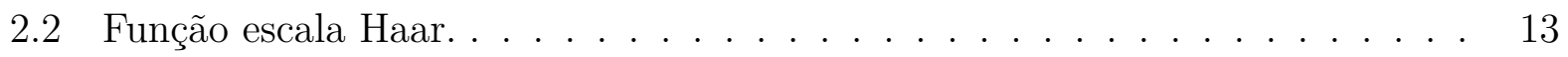

2.3 Ondaleta Haar. . . . . . . . . . . . . . . . . . . . . . . . . . . . . . . . . . . . . 13

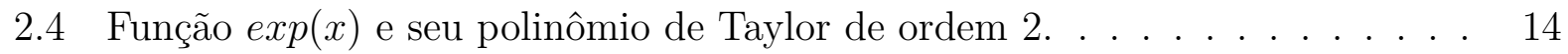

2.5 Aproximação de função por Kernel de Epachnikov . . . . . . . . . . . . . . . 17

$3.1 C_{\alpha}\left(u_{1}, u_{2}\right)$ cópula Normal $\ldots \ldots \ldots \ldots \ldots$

$3.2 c_{\alpha}\left(u_{1}, u_{2}\right)$ Densidade de cópula Normal . . . . . . . . . . . . . . . . 28

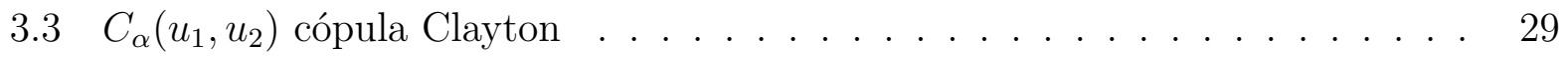

$3.4 c_{\alpha}\left(u_{1}, u_{2}\right)$ Densidade de cópula Clayton . . . . . . . . . . . . . . . . . 29

4.1 Retorno diário da HP e IBM . . . . . . . . . . . . . . . . . . . . . . . . . . . 32

4.2 Série temporal multivariada $\boldsymbol{y}_{t}=\left(y_{1 t}, y_{2 t}\right)^{\top}$ simulada usando as estimativas da Equação 4.3 . . . . . . . . . . . . . . . . . . . . . . . . . . . . 33

4.3 Cópula $C_{t}(u, u)$ para $u=0,01 ; 0,05 ; 0,25 ; 0,5 ; 0,75 ; 0,95 ; 0,99$. . . . . . . . 35

5.1 viés $s_{\theta}(t)$ e $E Q M_{\theta}(t)$ Caso Clayton . . . . . . . . . . . . . . . . . . . 50

5.2 viés $s_{\tau}(t)$ e $E Q M_{\tau}(t)$ Caso Clayton . . . . . . . . . . . . . . . . . . . . . . 51

5.3 viés $s_{\theta}(t)$ e $E Q M_{\theta}(t)$ Caso Normal . . . . . . . . . . . . . . . . . . . . . 53

5.4 viés $s_{\tau}(t)$ e $E Q M_{\tau}(t)$ Caso Normal . . . . . . . . . . . . . . . . . . . . . 55

5.5 Log retorno do Real e do Euro precificados em Dólares . . . . . . . . . . . . 56

5.6 Acf e pacf dos log retornos do Real e do Euro . . . . . . . . . . . . . . . 56

5.7 Quadrado do log retorno . . . . . . . . . . . . . . . . . . . . . 57

5.8 Acf e pacf dos quadrados dos log retornos . . . . . . . . . . . . . . . . . . 57

$5.9 \hat{\theta}_{t}$ para a cópula Clayton . . . . . . . . . . . . . . . . . 59

$5.10 \hat{\tau}$ de Kendall cópula Clayton . . . . . . . . . . . . . . . . . . . . . . . . . . 59 
xii LISTA DE FIGURAS 


\section{Lista de Tabelas}

5.1 viés $(\boldsymbol{u})$ para cópula Clayton $\ldots \ldots$. . . . . . . . . . . . . . . . . . 49

$5.2 \quad E Q M(\boldsymbol{u})$ para cópula Clayton $\ldots \ldots \ldots \ldots$. . . . . . . . . . . . . 49

5.3 viés $(\theta)$ e $\operatorname{EQM}(\theta)$ Cópula Clayton . . . . . . . . . . . . . . . . . . 51

5.4 viés $(\tau)$ e $\operatorname{EQM}(\tau)$ cópula Calyton $\ldots \ldots \ldots \ldots$. . . . . . . . . . . . 52

5.5 viés $(\boldsymbol{u})$ cópula Normal . . . . . . . . . . . . . . . . . . . . 53

$5.6 \quad E Q M(\boldsymbol{u})$ cópula Normal . . . . . . . . . . . . . . . . . . . . . . . . . . . . . 53

$5.7 \quad$ viés $(\theta)$ e $E Q M(\theta)$ cópula Normal . . . . . . . . . . . . . . . . . . . . . . . . 54

$5.8 \quad$ viés $(\tau)$ e $E Q M(\tau)$ cópula Normal . . . . . . . . . . . . . . . . . . . . . 55

5.9 AIC e BIC para o Ajuste das Cópulas . . . . . . . . . . . . . . . . . . . 58 


\section{Capítulo 1}

\section{Introdução}

Em várias situações da ciência e da vida moderna, o entendimento das relações entre fenômenos é relevante. Por exemplo, em finanças a cotação do dólar americano está relacionada à cotação do euro, entre outros fatores; na administração pública, as informações sobre o volume, o pico e a duração de enchentes são convenientes para as autoridades públicas em situações de contingência. Consequentemente, nota-se o mérito da compreensão da dependência entre fenômenos.

Ressaltamos ainda que em diversos campos do conhecimento, os eventos são mensurados ao longo do tempo. Por exemplo, podemos citar a análise de índices econômicos como a taxa de inflação, balança comercial e produção industrial (Harrison, 1996, Bollerslev, 1986); o estudo de indicadores em medicina como o índice da qualidade do ar, a taxa de mortalidade e número de doenças cardiopulmonares (Prescott, Cohen, Elton, Fowkes, e Agius, 1998 , Atkinson, Fuller, Anderson, Harrison, e Armstrong, 2010; Zeger, Irizarry, e Peng, 2006); a investigação das mudanças climáticas e suas consequências (Petit, Jouzel, Raynaud, Barkov, Barnola, Basile, Bender, Chappellaz, Davis, Delaygue, et al., 1999 ; Kovats, Edwards, Hajat, Armstrong, Ebi, e Menne, 2004).

Neste contexto, a partir da década de 1950, o estudo de dependência entre eventos tem ganhado crescente relevância, com destaque para a técnica denominada Cópula. Entre as áreas de aplicação, podemos citar: em finanças e mercado de seguros (Denuit, Dhaene, 
Goovaerts, e Kaas, 2006), (Klugman, Panjer, e Willmot, 2012); em saúde (Deb, Trivedi, e Zimmer, 2014; Zhao e Zhou, 2012); em hidrologia (Renard e Lang, 2007; Salvadori e De Michele, 2007; Shiau, Feng, e Nadarajah, 2007).

Um passo central na análise de risco é a construção de um modelo estatístico que retrata a aleatoriedade inerente à situação. Por muito tempo, modelos estatísticos em atuária e finanças basearam-se em suposições simplificadoras e sob essas suposições a distribuição normal domina o estudo de distribuições multivariadas. Entretanto, os gerenciadores de risco em companhias de seguro e finanças estão confrontando riscos mais complexos e múltiplos, portanto modelar dependência além da normalidade multivariada torna-se crucial. Um exemplo é o da escolha dos ativos na composição de um portfólio, envolve encontrar quantidade de ações de cada tipo que maximizam a utilidade esperada do investidor e, portanto, requer uma distribuição multivariada preditiva para os ativos que estão sendo considerados. A abordagem utilizando cópula facilita a construção da distribuição multivariada. A essência dessa abordagem é que uma distribuição conjunta de variáveis aleatórias pode ser expressa como uma função das distribuições marginais.

Adicionalmente, há situações em que os eventos são observados ao longo do tempo e desejamos avaliar a dependência entre os mesmos. Neste contexto, Silva Filho, Ziegelmann, e Dueker (2014) analisaram a dependência dinâmica entre os índices de mercado dos Estados Unidos (S\&P500), da Grã-Bretanha (FTSE100), do Brasil (IBOVESPA) e do México (PCMX) utilizando cópulas condicionais. Patton (2012) faz uma revisão das técnicas de cópulas no contexto de análise de séries temporais. Zhang e Guegan (2008) propõem o uso de modelos GARCH e cópula variante no tempo. Manner e Reznikova (2012) reúnem diferentes especificações presentes na literatura para cópula variante no tempo. Almeida e Czado (2012) propõem uma classe de modelos para cópula variante no tempo usando variáveis latentes e abordagem Bayesiana. Aloui, Hammoudeh, e Nguyen (2013) investigam a dependência entre o cotação do barril de petróleo e o mercado de ações do leste europeu. Ausin e Lopes (2010) apresentaram uma abordagem totalmente Bayesiana em que todos os parâmetros são 
estimados simultaneamente e um procedimento Bayesiano é introduzido para a estimação de medidas de dependências como VaR (Value-at-Risk) e CVaR (Conditional-Value-at-Risk). No entanto, notamos a carência de modelos semi-paramétricos usando abordagem Bayesiana para cópulas variantes no tempo.

Neste trabalho, propomos uma abordagem semi-paramétrica adaptando a proposta de cópula condicional em Abegaz, Gijbels, e Veraverbeke (2012) para cópulas variantes no tempo. Mais precisamente, cópulas variantes no tempo serão aproximadas por uma base de funções e estimamos os coeficientes dessa aproximação usando inferência Bayesiana. Neste trabalho, usamos as bases de funções: ondaletas Haar, polinômios de Taylor e kernel smoother com núcleo Epanechnikov. O texto é organizado como segue: no Capítulo 1 fazemos uma introdução; no Capítulo 2 revisamos alguns conceitos básicos; no Capítulo 3 apresentamos os conceitos de cópula; no Capítulo 4 apresentamos o conceito de cópulas variantes no tempo; Capítulo 5 expomos um estudo de simulação e aplicações aos dados reais; e, finalmente, no Capítulo 6 fazemos algumas considerações finais e tópicos para estudos futuros. 


\section{Capítulo 2}

\section{Conceitos básicos}

Neste capítulo, apresentamos alguns conceitos para o entendimento de séries temporais, ondaletas, polinômios de Taylor e Kernel smoother com núcleo Epanechnikov. Ressaltamos que não pretendemos ser uma referência exaustiva e completa. Em cada seção apresentamos referências para estudos complementares.

\subsection{Séries temporais}

Um processo estocástico tem sua definição formal como segue:

\section{Definição 2.1. Processo estocástico}

Seja $T$ um conjunto arbitrário. Um processo estocástico é uma família $X=\left\{X_{t}: t \in T\right\}$, tal que, para cada $t \in T, X_{t}$ é uma variável aleatória.

Nestas condições, um processo estocástico é uma família de variáveis aleatórias (v.a.), definidas num mesmo espaço de probabilidades $(\Omega, \mathcal{A}, \mathcal{P})$. O conjunto $T$ é normalmente tomado como o conjunto dos inteiros $\mathbb{Z}=\{0, \pm 1, \pm 2, \ldots\}$ ou o conjunto dos reais $\mathbb{R}$. Também, para cada $t \in T, X_{t}$ será uma variável aleatória real. Como, para $t \in T, X_{t}$ é uma v.a. definida sobre $\Omega$, na realidade $X_{t}$ é uma função de dois argumentos, $X(t, \omega), t \in T, \omega \in \Omega$.

Para cada $\omega \in \Omega$ fixado, obtemos uma função em t, ou seja, uma realização ou trajetória 
do processo, ou uma série temporal. Para mais detalhes ver (Morettin e Toloi, 2006).

Informalmente, uma série temporal $X_{t}$ é uma coleção de variáveis aleatórias indexadas no tempo. A função média e a função de autocovariância de $X_{t}$ são definidas como $\mu(t)=$ $E\left\{X_{t}\right\}$, e $\gamma\left(t_{1}, t_{2}\right)=E\left\{X_{t_{1}}, X_{t_{2}}\right\}-E\left\{X_{t_{1}}\right\} E\left\{X_{t_{2}}\right\}, t_{1}, t_{2} \in T$, respectivamente. Em particular se $t_{1}=t_{2}=t$, temos a variância do processo $X_{t}, \gamma(t, t)=\operatorname{Var}\left(X_{t}\right)=V(t)=$ $E\left\{X_{t}^{2}\right\}-E\left\{X_{t}\right\}^{2}$.

\subsubsection{Estacionariedade}

Um dos conceitos fundamentais em análise de séries temporais é o de estacionariedade.

Definição 2.2. Um processo estocástico $X=\left\{X_{t}, t \in T\right\}$ diz-se estritamente estacionário se todas as distribuições finito-dimensionais permanecem as mesmas sob translações no tempo, ou seja,

$$
F\left(x_{1}, x_{2}, \ldots, x_{n} ; t_{1}+\tau, \ldots, t_{n}+\tau\right)=F\left(x_{1}, \ldots, x_{n} ; t_{1}, \ldots, t_{n}\right)
$$

para quaisquer $t_{1}, \ldots, t_{n}, \tau$ de $\boldsymbol{T}$.

Isso significa, em particular, que todas as distribuições unidimensionais são invariantes sob translação no tempo, logo a média $\mu(t)$ e a variância $V(t)$ são constantes, isto é,

$$
\mu(t)=\mu, \quad V(t)=\sigma^{2}, \forall t \in \boldsymbol{T} .
$$

Definição 2.3. Um processo estocástico $X=\left\{X_{t}, t \in T\right\}$ é dito fracamente estacionária ou estacionário de segunda ordem (ou em sentido amplo) se e somente se

$i-E\left\{X_{t}\right\}=\mu(t)=\mu$, constante para todo $t \in T$;

$i i-E\left\{X_{t}^{2}\right\}<\infty$, para todo $t \in T$;

iii $-\gamma\left(t_{1}, t_{2}\right)=\operatorname{Cov}\left(X_{t_{1}}, X_{t_{2}}\right)$ é uma função de $\left|t_{1}-t_{2}\right|$.

Na prática, nosso interesse é caracterizar o processo estocástico por meio dos momentos de primeira e segunda ordens. 


\subsubsection{Ruído branco}

Dizemos que uma sequência de variáveis aleatórias $X_{t}$ é um ruído branco se as variáveis aleatórias são não correlacionadas, isto é, $\operatorname{Cov}\left(X_{t}, X_{s}\right)=0, t \neq s$. Tal processo será estacionário se $E\left(X_{t}\right)=\mu_{x}$ e $\operatorname{Var}\left(X_{t}\right)=\sigma_{x}^{2}, \forall t$. Na Figura 2.1 podemos ver um exemplo de uma série independente e identicamente distribuída (i.i.d.) normalmente distribuída com média zero e variância $\sigma^{2}=1$.

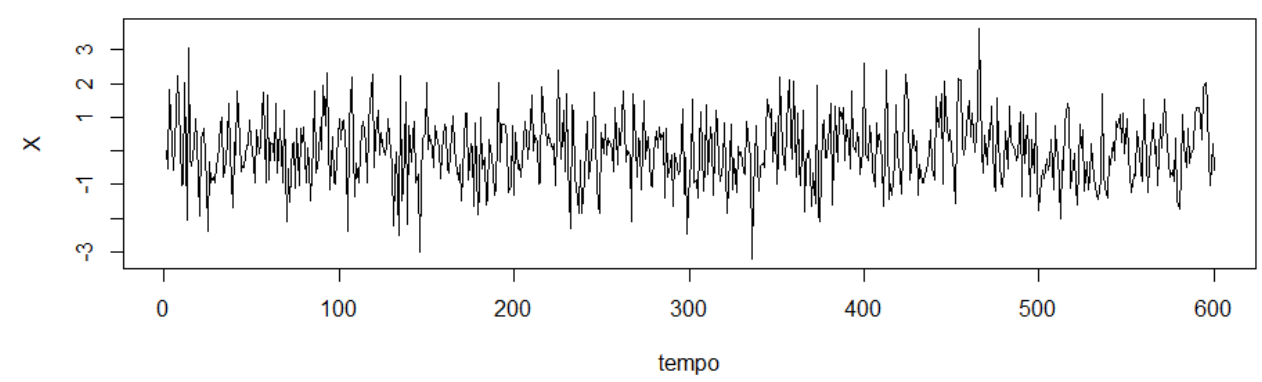

Figura 2.1: Ruído branco, i.i.d.N(0,1)

Definição 2.4. Um processo estocástico $X=\left\{X_{t}, t \in T\right\}$ é dito Gaussiano se para qualquer conjunto $t_{1}, t_{2}, t_{3}, \ldots, t_{n}$ de $T$, as v.a. $X\left(t_{1}\right), X\left(t_{2}\right), \ldots, X\left(t_{n}\right)$ têm distribuição normal $n$ variada.

Um processo Gaussiano será determinado pelas médias e covariâncias; em particular, se ele for fracamente estacionário, ele será estritamente estacionário.

\subsubsection{Função de autocovariância e função de autocorrelação}

Definição 2.5. A função de autocovariância (facv) é definida como o segundo momento produto

$$
\gamma_{X}(s, t)=\operatorname{cov}\left(X_{s}, X_{t}\right)=E\left[\left(X_{s}-\mu_{s}\right)\left(X_{t}-\mu_{t}\right)\right]
$$

para todo $s, t \in T$. Quando não houver confusão a respeito da série em questão escreveremos $\gamma_{X}(s, t)$ como $\gamma(s, t)$. 
A função de autocovariância (facv) mede a dependência linear entre dois pontos observados em tempos diferentes dentro de uma mesma série. A autocovariância é a média do produto cruzado relativo a distribuição conjunta $F\left(X_{s}, X_{t}\right)$. É importante lembrar da estatística clássica que se $\gamma(s, t)=0, X_{s}$ e $X_{t}$ são não linearmente relacionados, no entanto, pode haver uma estrutura de dependência entre elas. Todavia, se $X_{s}$ e $X_{t}$ são normais bivariadas, $\gamma(s, t)=0$, garante a sua independência (Shumway e Stoffer, 2013).

\section{Propriedades da facv}

Seja $\left\{X_{t}, t \in Z\right\}$ um processo estacionário real discreto, de média zero e facv $\gamma_{\tau}=$ $E\left\{X_{t} X_{t+\tau}\right\}$. A facv satisfaz as propriedades $\}^{1}$ de (i) a (iv) a seguir:

(i) $\gamma_{0}>0$,

(ii) $\gamma_{-\tau}=\gamma_{\tau}$,

(iii) $\left|\gamma_{\tau}\right| \leq \gamma_{0}$

(iv) $\gamma_{\tau}$ é não negativa definida, no sentido que

$$
\sum_{j=1}^{N} \sum_{k=1}^{N} a_{j} a_{k} \gamma_{\tau_{j}-\tau_{k}} \geq 0,
$$

para quaisquer números reais $a_{1}, a_{2}, \ldots, a_{n}$, e $\tau_{1}, \tau_{2}, \ldots, \tau_{n}$.

A função de autocorrelação mede o grau de correlação entre $X_{t}$ e $X_{t+\tau}$ e é definida da seguinte maneira:

Definição 2.6. função de autocorrelação (acf) é dada por

$$
\rho_{\tau}=\frac{\gamma(t, t+\tau)}{\sqrt{\gamma(t, t) \gamma(t+\tau, t+\tau)}}=\frac{\gamma_{\tau}}{\gamma_{0}} .
$$

\footnotetext{
${ }^{1}$ As demonstrações destas propriedades estão em Morettin e Toloi (2006) pág 25. Morettin observa ainda que dada uma função $\gamma_{\tau}$ tendo a propriedade (iv), existe um processo estocástico $X_{t}$ tendo $\gamma_{\tau}$ como facv e que na verdade $X_{t}$ pode ser tomado como Gaussiano.

É típico da facv de um processo estacionário tender a zero quando $|\tau| \rightarrow \infty$
} 
Definição 2.7. A medida de correlação cruzada entre duas séries temporais $X_{t}$ e $Y_{t}$ é dada por

$$
\rho_{x y}(s, t)=\frac{\gamma_{x y}(s, t)}{\sqrt{\gamma_{x}(s, s) \gamma_{y}(t, t)}} .
$$

\subsection{Ondaletas}

A ideia, tanto na análise de séries de Fourier, na aproximação de Taylor, na análise de ondaletas ou em qualquer outra base, é aproximar uma função por uma combinação de senos e cossenos, polinômios ortogonais, ou outras bases de funções adequadas. Particularmente no caso de ondaletas, desejamos aproximar uma função $f \in L^{2}(\mathbb{R})=\{f: \mathbb{R} \rightarrow$ $\left.\left.\mathbb{R}\left|\int_{-\infty}^{\infty}\right| f(u)\right|^{2} d u<\infty\right\}$ por uma combinação linear de dilatações binárias $2^{j}$ e translações diádicas $k 2^{-j}$ de funções $\phi(x)$, chamada de função escala ou ondaleta pai, e / ou de funções $\psi(x)$, chamada de função ondaleta ou ondaleta mãe.

O campo de aplicação de ondaletas é extenso e inclui processamento de sinais, tratamento de imagens, compressão de dados, estimação de densidade, regressão não paramétrica e outros. Como referência podemos citar Boggess e Narcowich (2009), Daubechies et al. (1992), Meyer e Ryan (1993), Morettin (2014), Meyer e Salinger (1995) e Chui (2014).

Nesta seção, expomos as ideias básicas sobre ondaletas, começando pela Seção 2.2.1 onde descrevemos a função ondaleta, também chamada de ondaleta mãe, e a função escala, também denominada ondaleta pai.

\subsubsection{Função Escala e Função Ondaleta}

Ondaletas são funções que satisfazem certas propriedades. Elas podem ser suaves ou não, simétricas ou não e podem ter expressões matemáticas simples ou não.

Considere o espaço $L^{2}(\mathbb{R})=\left\{f:\left.\mathbb{R} \rightarrow \mathbb{R}\left|\int_{\mathbb{R}}\right| f(u)\right|^{2} d u<\infty\right\}$ das funções quadrado integráveis em $\mathbb{R}$. A ideia é considerar dilatações (ou compressões) e translações de uma 
função $\psi$ de modo a cobrir todo $\mathbb{R}$. Mais precisamente, consideramos

$$
\psi_{j, k}(t)=2^{\frac{j}{2}} \psi\left(2^{j} t-k\right)
$$

em que $\left\{\psi_{j, k}(t) \mid j, k \in \mathbb{Z}\right\}$ é uma base para $L^{2}(\mathbb{R})$ e $\psi$ é chamada de ondaleta mãe ou função ondaleta.

Suponha que $\left\{\psi_{j, k}(t) \mid j, k \in \mathbb{Z}\right\}$ é uma base ortonormal gerada por $\psi 2$, isto é,

$$
\left\langle\psi_{j, k}, \psi_{l, m}\right\rangle=\delta_{j, l} \delta_{k, m}, \quad j, k, l, m \in \mathbb{Z}
$$

em que $\delta_{i, j}=\left\{\begin{array}{l}1, \text { se } i=j, \\ 0, \text { caso contrário, }\end{array}\right.$

então para qualquer $f(t) \in L^{2}(\mathbb{R})$,

$$
f(t)=\sum_{j=-\infty}^{\infty} \sum_{k=-\infty}^{\infty} d_{j, k} \psi_{j, k}(t),
$$

em que a convergência é considerada em média quadrática. Adicionalmente, a série na equação (2.1) é chamada de série de ondaletas de $f(t)$ e $d_{j, k}, j, k \in \mathbb{Z}$ são denominados coeficientes de ondaletas e são calculados por

$$
\begin{aligned}
d_{j, k} & =\left\langle f(t), \psi_{j, k}(t)\right\rangle \\
& =\int_{-\infty}^{\infty} f(u) \psi_{j, k}(u) d u
\end{aligned}
$$

Neste contexto de ortogonalidade e analogamente à análise de série de Fourier, a relação de

\footnotetext{
${ }^{2}$ Meyer (1985) demonstrou que existe uma função $\psi(x)$ tal que $\left\{\psi_{j, k}(x) \mid j, k \in \mathbb{Z}\right\}$ é uma base ortonormal para $L^{2}(\mathbb{R})$.
} 
Parseval é válida. Mais precisamente,

$$
\int_{-\infty}^{\infty} f^{2}(u) d u=\sum_{j=-\infty}^{\infty} \sum_{k=-\infty}^{\infty} d_{j, k}^{2} .
$$

As seguintes propriedades para a ondaleta mãe são válidas:

i. $\int_{-\infty}^{\infty} \psi(t) d t=0$,

ii. $\int_{-\infty}^{\infty}|\psi(t)| d t<\infty$

iii. $\int_{-\infty}^{\infty}|\psi(t)|^{2} d t=1$,

iv. Existe $r \in\{j \in \mathbb{Z} \mid j \geq 1\}$ tal que

$$
\begin{aligned}
& \int_{-\infty}^{\infty} t^{j} \psi(t) d t=0, \quad j=0,1, \ldots, r-1 \\
& \mathrm{e} \int_{-\infty}^{\infty}\left|t^{r} \psi(t)\right| d t<\infty
\end{aligned}
$$

O valor de $r$ na propriedade iv. está ligado ao grau de regularidade (suavidade) de $\psi$ : quanto maior o valor de $r$, mais suave será $\psi$. Algumas ondaletas têm suporte compacto, que é uma propriedade desejável. Nem todas as ondaletas geram sistemas ortogonais. Se a ondaleta tem suporte compacto, o valor de $r$ está relacionado ao seu suporte (vide Härdle, Kerkyacharian, Tsybakov, e Picard, 1998, para mais detalhes). Poderíamos ter considerado translações (ou compressões) e dilatações de uma função $\phi$, denominada função escala ou ondaleta pai, para aproximar $f(t)$. Uma outra maneira de gerar ondaletas é pela função escala, ou ondaleta pai $\phi$, que é a solução da equação

$$
\phi(t)=\sqrt{2} \sum_{k} l_{k} \phi(2 t-k)
$$

Essa função gera uma família ortonormal em $L^{2}(\mathbb{R}): \phi_{j, k}(t)=2^{j} \phi\left(2^{j} t-k\right), j, k \in \mathbb{Z}$, nessas 
condições, $\psi$ pode ser obtida de $\phi$ por

$$
\psi(t)=\sqrt{2} \sum_{k} h_{k} \phi(2 t-k)
$$

em que $h_{k}$ e $l_{k}$ são associadas pela igualdade $h_{k}=(-1)^{k} l_{1-k}$, chamada de quadrature mirror filter relation. Além disso, temos que

$$
\begin{aligned}
l_{k} & =\sqrt{2} \int_{-\infty}^{\infty} \phi(t) \phi(2 t-k) d t, \\
h_{k} & =\sqrt{2} \int_{-\infty}^{\infty} \psi(t) \phi(2 t-k) d t,
\end{aligned}
$$

em que $l_{k}$ é denominado filtro passo-baixo e $h_{k}$ é chamado de filtro passo-alto.

Considere o sistema ortonormal $\left\{\phi_{j_{0}, k}(t), \psi_{j, k}(t), j, k \in \mathbb{Z}, j \geq j_{0}\right\}$ de tal sorte que podemos escrever, para $f(t) \in L^{2}(\mathbb{R})$,

$$
f(t)=\sum_{k} c_{j_{0}, k} \phi_{j_{0}, k}(t)+\sum_{j>j_{0}} \sum_{k} d_{j, k} \psi_{j, k}(t)
$$

em que

$$
\begin{aligned}
c_{j_{0}, k} & =\int_{-\infty}^{\infty} f(t) \phi_{j_{0}, k}(t) d t \\
d_{j, k} & =\int_{-\infty}^{\infty} f(t) \psi_{j, k}(t) d t
\end{aligned}
$$

Exemplo 2.1. O exemplo mais antigo e mais simples é o caso Haar, introduzido inicialmente por Alfred Haar no trabalho Haar (1909). 
A ondaleta Haar é descrita por

$$
\psi(t)= \begin{cases}1, & 0 \leq t<\frac{1}{2} \\ -1, & \frac{1}{2} \leq t<1 \\ 0, & \text { caso contrário }\end{cases}
$$

e a função escala é dada por $\phi(t)= \begin{cases}1, & t \in[0,1) \\ 0, & \text { caso contário. }\end{cases}$

Note que $\psi(t)$ e $\phi(t)$ tem suporte compacto e

$$
\phi(t)=\phi(2 t)+\phi(2 t-1)=\frac{1}{\sqrt{2}} \sqrt{2} \phi(2 t)+\frac{1}{\sqrt{2}} \sqrt{2} \phi(2 t-1)
$$

e

$$
\psi(t)=\phi(2 t)-\phi(2 t-1)=\frac{1}{\sqrt{2}} \sqrt{2} \phi(2 t)-\frac{1}{\sqrt{2}} \sqrt{2} \phi(2 t-1)
$$

ou seja, $l_{0}=l_{1}=\sqrt{\frac{1}{2}}, h_{0}=l_{0}$ e $h_{1}=-l_{1}$. É possível verificar que $\left\{\psi_{j, k}(t) \mid j, k \in \mathbb{Z}\right\}$ é uma base ortonormal para $L^{2}(\mathbb{R})$, para detalhes ver Meyer (1985) e Morettin (2014). Na Figura 2.2 mostramos o gráfico da função escala e na Figura 2.3 a ondaleta Haar.

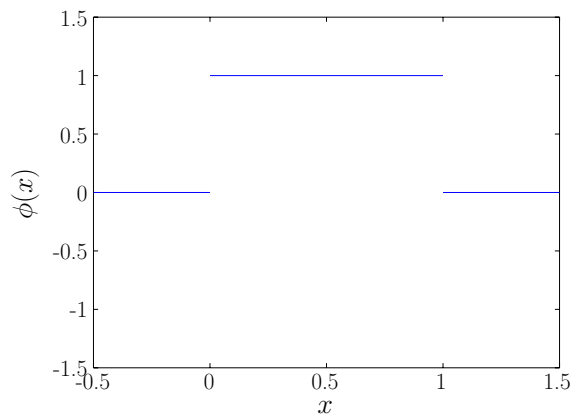

Figura 2.2: Função escala Haar.

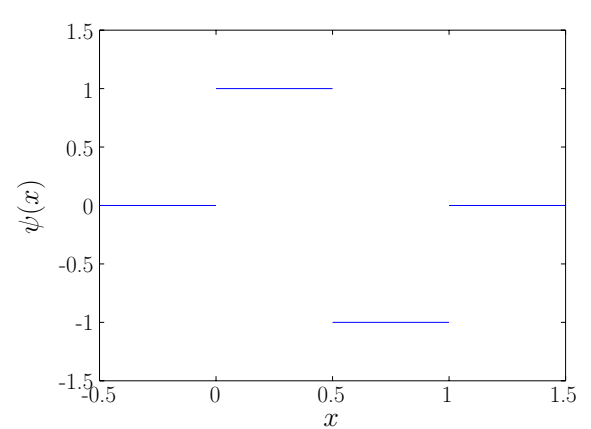

Figura 2.3: Ondaleta Haar. 


\subsection{Polinômio de Taylor}

Considere o conjunto das funções $n$-deriváveis com a $n$-ésima derivada contínua, denotado por $C^{n}=\left\{f: I \longrightarrow \mathbb{R} \mid f^{(n)}(x)\right.$ é contínua $\}$. A ideia do polinômio de Taylor é usar as derivadas para construir uma aproximação para $f \in C^{n}$. Mais precisamente, seja $f \in C^{n}$ e $P_{n}(x)$ um polinômio de grau $n$ satisfazendo $P_{k}\left(x_{0}\right)=f^{(k)}\left(x_{0}\right), k=0,1,2, \ldots, n$. Podemos concluir que $P_{n}(x)$ terá a seguinte forma:

$$
P_{n}(x)=f\left(x_{0}\right)+\frac{f^{(1)}\left(x_{0}\right)}{1 !}\left(x-x_{0}\right)+\frac{f^{(2)}\left(x_{0}\right)}{2 !}\left(x-x_{0}\right)^{2}+\cdots+\frac{f^{(n)}\left(x_{0}\right)}{n !}\left(x-x_{0}\right)^{n}
$$

o qual é chamado de polinômio de Taylor de ordem $n$ de $f(x)$, ao redor de $\left(x_{0}\right)$.

Exemplo 2.2. O polinômio de Taylor de ordem 2 ao redor do zero $\left(x_{0}=0\right)$ da função $f(x)=e^{x}$ é $P_{2}(x)=1+x+\frac{x^{2}}{2}$,

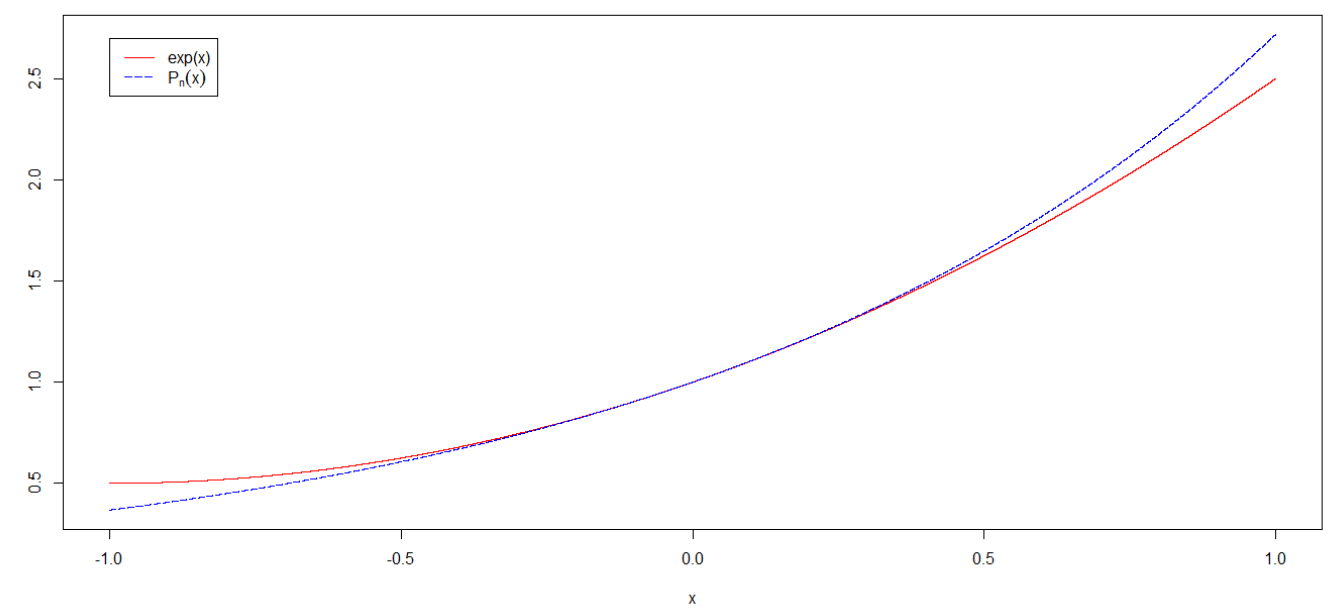

Figura 2.4: Função $\exp (x)$ e seu polinômio de Taylor de ordem 2.

Na Figura 2.4 mostramos o polinômio $P_{2}(x)$ e $f(x)=e^{x}, x \in(-1,1)$. Note que as duas curvas estão bastante próximas.

Teorema 2.3 (Teorema de Taylor). Sejam $n \in \mathbb{N}$ e $f: I \longrightarrow \mathbb{R}$ definida em um intervalo $I$ tal que suas derivadas $f(x), f^{(1)}(x), f^{(2)}(x), \ldots, f^{(n)}(x)$ são continuas e $f^{(n+1)}\left(x_{0}\right)$ existe em 
I. Então para todo $x \in I$, existe $c \in I$ tal que

$f(x)=f\left(x_{0}\right)+\frac{f^{(1)}\left(x_{0}\right)}{1 !}\left(x-x_{0}\right)+\frac{f^{(2)}\left(x_{0}\right)}{2 !}\left(x-x_{0}\right)^{2}+\cdots+\frac{f^{(n)}\left(x_{0}\right)}{n !}\left(x-x_{0}\right)^{n}+\frac{f^{(n+1)}(c)}{(n+1) !}\left(x-x_{0}\right)^{n+1}$,

em que $x_{0} \in I$.

Demonstração. Vide Bartle e Sherbert (1992) para detalhes e demonstração.

Note que

$$
f(x)=P_{n}(x)+R_{n}(x)
$$

em que

$$
R_{n}(x)=\frac{f^{(n+1)}(c)}{(n+1) !}\left(x-x_{0}\right)^{n+1}
$$

é chamada de resto de Lagrange para algum $c$ entre $x$ e $x_{0}$. Além disso, observe que se $\left|f^{(n+1)}(x)\right| \leq M, \forall x \in I$ e existe $r>0$ tal que $I \subset\left(x_{0}-r, x_{0}+r\right)$, então

$$
\begin{aligned}
\left|R_{n}(x)\right| & =\left|f^{(n+1)}(c) \frac{\left(x-x_{0}\right)^{n+1}}{(n+1) !}\right| \\
& \leq \frac{M}{(n+1) !}\left|x-x_{0}\right|^{n+1} \\
& \leq M \frac{r^{n+1}}{(n+1) !}
\end{aligned}
$$

Como

$$
\lim _{n \rightarrow \infty} \frac{r^{n+1}}{(n+1) !}=0,
$$

temos que

$$
\lim _{n \longrightarrow \infty}\left|R_{n}(x)\right|=0, \forall x \in I .
$$


Ou seja, para uma função com suporte compacto e todas derivadas contínuas, o polinômio de Taylor converge para esta função quando aumentamos a ordem de $P_{n}(x)$. Observe que podemos reescrever o polinômio de Taylor de ordem $n$ no seguinte formato

$$
P_{n}(x)=a_{0}+a_{1} x+\cdots+a_{n} x_{n}
$$

Neste trabalho, aproximamos o parâmetro da cópula variante no tempo por $P_{n}(x)$ estimando os coeficientes $a_{0}, a_{1}, \ldots, a_{n}$ usando algoritmos MCMC conforme descrito no Capítulo 4.

\subsection{Métodos de suavização por Kernel}

Nesta seção, descrevemos uma técnica que permite estimar uma função real $h(u), u \in$ $[0,1]$, ajustando um modelo diferente mas simples para cada ponto $u_{0} \in[0,1]$. Para este fim, usamos observações próximas de $u_{0}$ para ajustar um modelo simples e a função resultante $\hat{h}(u)$ é suave em $[0,1]$. Para localizar as observações próximas usamos uma função peso ou kernel $K_{\lambda}\left(u_{0}, u_{i}\right)$ que atribui um peso para cada valor $h_{i}=h\left(x_{i}\right)$ baseado na distância de $x_{i}$ e $x_{0}$. O kernel $K_{\lambda}$ é indexado pelo parâmetro $\lambda$, chamado bandwidth, que estabelece a largura da vizinhança de $u_{0}$ usado no modelo simples.

Mais precisamente, usamos a abordagem de Nadaraya-Watson para estimar $h(u)$

$$
\hat{h}\left(u_{0}\right)=\frac{\sum_{i=1}^{N} K_{\lambda}\left(u_{0}, u_{i}\right) h_{i}}{\sum_{i=1}^{N} K_{\lambda}\left(u_{0}, u_{i}\right)}, \quad u_{0} \in[0,1]
$$

em que $u_{1}, \ldots, u_{N}$ são pontos igualmente espaçado com $u_{1}=0$ e $u_{N}=1 ; h\left(u_{1}\right)=h_{1}, \ldots, h\left(u_{N}\right)=$ $h_{N}$;

$$
K_{\lambda}\left(u_{0}, u_{i}\right)=D\left(\frac{\left|u_{0}-u_{i}\right|}{\lambda}\right)
$$


é o kernel Epanechnikov com

$$
D(u)= \begin{cases}\frac{3}{4}(1-u)^{2}, & \text { se }|u| \leq 1 \\ 0, & \text { caso contrário. }\end{cases}
$$

Na Figura 2.5 temos um exemplo da estimação de função por Kernel de Epanechnikov. A curva azul representa a curva que desejamos estimar e a vermelha a estimação por Kernel, usando $\lambda=0,2$.

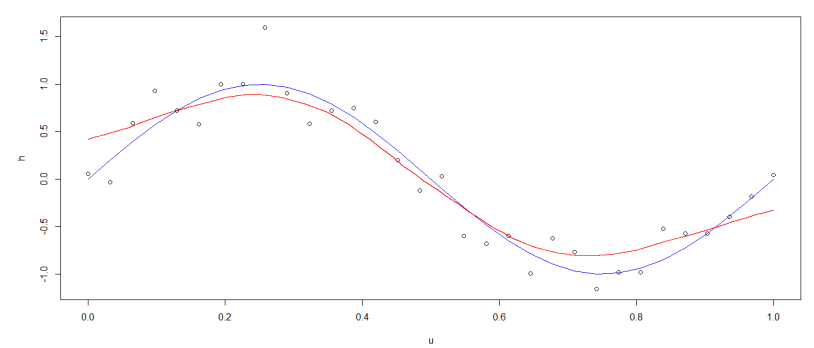

Figura 2.5: Aproximação de função por Kernel de Epachnikov

Para mais detalhes, consulte, por exemplo, Friedman, Hastie, e Tibshirani (2001); Wand e Jones (1994); Horová, Koláček, e Zelinka (2012).

Neste trabalho fixamos $\lambda=0.2$ e desejamos estimar o vetor $\left(h_{1}, \ldots, h_{N}\right)^{\top}$ usando método MCMC conforme descrito no Capítulo 4. 


\section{Capítulo 3}

\section{Cópula}

O estabelecimento de estrutura de dependência entre duas ou mais variáveis aleatórias é um objetivo importante, e as cópulas podem ser úteis, a fim de realizar esta tarefa. Uma cópula é uma função que liga as distribuições marginais das variáveis aleatórias à sua conjunta. Em muitas situações as distribuições marginais são conhecidas (ou podem ser estimadas) e a distribuição conjunta é desconhecida, ou pode ser difícil de estimar.

Há muitas aplicações de cópulas para finanças. Um importante problema na econometria financeira é modelar os retornos dos ativos com o objetivo de mensurar as medidas de risco, tais como o valor em risco (VaR). Uma suposição básica que é frequentemente usada é que os retornos são gaussianos, mas é bem conhecido que tais retornos têm caudas pesadas e apresentam alta curtose. Além disso, para calcular essas medidas de risco, é necessário calcular a matriz de covariância de uma carteira contendo um grande número de ativos, assim modelar um vetor de retornos é um verdadeiro desafio. Estas dificuldades levaram à utilização de cópulas em Finanças. Algumas referencias recentes são, Embrechts, Lindskog, e McNeil (2001), Joe (1997), Bouyé, Durrleman, Nikeghbali, Riboulet, e Roncalli (2000), Cherubini e Luciano (2001), Embrechts, Klüppelberg, e Mikosch (2013). Scaillet e Fermanian (2002), Fermanian e Wegkamp 2004) 


\subsection{Definições e propriedades básicas de Cópulas}

O conceito de cópula foi introduzido por Sklar (1959) e desde então a teoria e as aplicações desenvolveram-se em um ritmo rápido.

\subsubsection{Propriedades das distribuições}

Seja $X$ uma variável aleatória com função de distribuição $F_{X}(x)=\operatorname{Pr}(X \leq x)$. Por simplicidade assuma que $F_{X}$ é contínua e diferenciável. A função de densidade $f_{X}(x)$ é definida por

$$
F_{X}(x)=\int_{-\infty}^{x} f_{X}(z) d z
$$

e portanto $f_{x}=F_{X}^{\prime}$.

Seja

$$
F_{X}^{-1}(\alpha)=\inf \left\{x \mid F_{X}(x) \geq \alpha\right\}
$$

para $\alpha \in(0,1)$. Temos os seguintes resultados:

- $F_{X}(X) \sim U(0,1)$, em que $U(0,1)$ denota uma variável aleatória uniformemente distribuída em $(0,1)$.

- Se $U \sim U(0,1)$ então $F_{X}^{-1}(U) \sim F_{X}$.

Seja $X$ e $Y$ variáveis aleatórias com funções de distribuições marginais $F_{X}$ e $F_{Y}$, respectivamente, então a função de distribuição conjunta é dada por

$$
F_{X Y}(x, y)=\operatorname{Pr}(X \leq x, Y \leq y)
$$

Em geral, as funções de distribuições marginais podem ser recuperadas da função de distribuição conjunta via

$$
F_{X}(x)=F_{X Y}(x, \infty), F_{Y}(y)=F_{X Y}(\infty, y)
$$


A densidade conjunta $f_{X Y}$ é definida por

$$
f_{X Y}(x, y)=\frac{\partial^{2}}{\partial x \partial y} F_{X Y}(x, y)
$$

As variáveis aleatórias $X$ e $Y$ são independentes se e somente se,

$$
F_{X Y}(x, y)=F_{X}(x) F_{Y}(y)
$$

para todos os valores de $x$ e $y$.

\subsubsection{Cópulas e Teorema de Sklar}

Uma copula bivariada é uma função de distribuição bivariada $C$ definida em $I^{2}=[0,1] \times$ $[0,1]$ com distribuições marginais uniformes, isto é,

$$
C(u, v)=\mathrm{P}(U \leq u, V \leq v)
$$

em que $U, V \sim U(0,1)$. Uma copula satisfaz as seguintes propriedades:

- $C(u, 0)=C(0, v)=0, C(1, v)=v, C(u, 1)=u$ para todo $u, v \in[0,1]$.

- $0 \leq C(u, v) \leq 1$.

- Para todo $u_{1} \leq u_{2}, v_{1} \leq v_{2}$, e $u_{1}, u_{2}, v_{1}, v_{2} \in[0,1]$, temos: $C\left(u_{1}, v_{1}\right)-C\left(u_{2}, v_{1}\right)-$ $C\left(u_{1}, v_{2}\right)+C\left(u_{2}, v_{2}\right) \geq 0$.

A última propriedade garante que $\operatorname{Pr}\left(u_{1} \leq U \leq u_{2}, v_{1} \leq V \leq v_{2}\right) \geq 0$.

A ideia de uma cópula é separar a função de distribuição conjunta $F_{X Y}$ em uma parte que descreve a dependência entre $X$ e $Y$ e partes que descrevem somente os comportamentos marginais. Mais precisamente, considere a função de distribuição conjunta $(U, V)$ como sendo 
a cópula $C$. Então temos

$$
\begin{aligned}
F_{X Y}(x, y) & =P(X \leq x, Y \leq y) \\
& =P\left(F_{X}(X) \leq F_{X}(x), F_{Y}(Y) \leq F_{Y}(y)\right) \\
& =C\left(F_{X}(x), F_{Y}(y)\right)
\end{aligned}
$$

portanto a função de distribuição conjunta $F_{X Y}$ pode ser descrita pelas marginais $F_{X}, F_{Y}$ e a cópula $C$. A cópula $C$ captura a estrutura de dependência entre $X$ e $Y$.

Teorema 3.1 (Teorema de Sklar:). Seja $F_{X Y}$ uma função de distribuição conjunta com marginais $F_{X}$ e $F_{Y}$. Então existe uma copula $C$ tal que para todos $x, y \in[-\infty, \infty]$,

$$
F_{X Y}(x, y)=C\left(F_{X}(x), F_{Y}(y)\right)
$$

Se $F_{X}$ e $F_{Y}$ são contínuas, então a cópula $C$ é única. Caso contrário, $C$ está definida unicamente em $\operatorname{Im} F_{X} \times I m F_{Y}$. Reciprocamente, se C é uma cópula e $F_{X}$ e $F_{Y}$ são funções de distribuições univariadas, então $F_{X Y}$ definida em (3.1) é a função de distribuição conjunta com marginais $F_{X}$ e $F_{Y}$.

O teorema de Sklar, Sklar (1959), mostra que a cópula associada com uma função de distribuição bivariada $F_{X Y}$ liga às marginais $F_{X}$ e $F_{Y}$ com estrutura de dependência unicamente determinada por $F_{X Y}$. Finalmente, é usualmente convencionado que a cópula de $X$ e $Y$ é a função de distribuição conjunta $C$ de $F_{X}(X)$ e $F_{Y}(Y)$. Para o caso contínuo, a densidade de cópula é definida por

$$
c\left(u_{1}, u_{2}\right)=\frac{\partial^{2} C\left(u_{1}, u_{2}\right)}{\partial u_{1} \partial u_{2}} .
$$

Seja $F_{X Y}$ a função de distribuição conjunta de $F_{X}$ e $F_{Y}$ definida em (3.1), então, usando 
a regra da cadeia, a densidade conjunta de $X$ e $Y$ pode ser recuperada utilizando

$$
\begin{aligned}
f_{X Y}(x, y) & =\frac{\partial^{2} F_{X Y}(x, y)}{\partial x \partial y} \\
& =\frac{\partial^{2} C\left(F_{X}(x), F_{Y}(y)\right)}{\partial x \partial y} \frac{\partial F_{X}}{\partial x} \frac{\partial F_{Y}}{\partial y} \\
& =c\left(F_{X}(x), F_{Y}(y)\right) f_{X}(x) f_{Y}(y) .
\end{aligned}
$$

O resultado acima mostra que é sempre possível especificar uma densidade bivariada utilizando as densidades marginais e a densidade de cópula.

\subsubsection{Os Limites de Fréchet-Hoeffding para distribuições conjun- tas}

Ao se trabalhar com cópulas, três resultados são fundamentais, além do teorema de Sklar. As cópulas são limitadas superiormente e inferiormente e damos a esses limites o nome de Limites de Fréchet-Hoeffding. Isso se dá graças aos trabalhos de Wassily Hoeffding e posteriormente aos de Maurice René Fréchet que chegam ao mesmo resultado independentemente (Nelsen, 2007, pág. 2). Além dos limites uma outra cópula importante é a cópula produto ou cópula independente. Consideremos $\boldsymbol{u}=\left(u_{1}, u_{2}\right)$ tais que $\left(u_{1}, u_{2}\right) \in[0,1]^{2}$, em que $u_{1}$ e $u_{2}$ são transformações integrais de $F_{X}$ e $F_{Y}$, respectivamente. A copula produto, o limite inferior e o limite superior de Fréchet-Hoeffding são dados da seguinte forma:

a) Se $X$ e $Y$ são independentes então, sua cópula satisfaz

$$
C_{I}(\boldsymbol{u})=C_{I}\left(u_{1}, u_{2}\right)=u_{1} u_{2}
$$

e é chamada de cópula independente ou ainda cópula produto.

b) Suponha que $X$ e $Y$ são variáveis aleatórias perfeitamente negativamente dependentes. 
Isso ocorre se $Y=T(X)$ e $T$ é uma transformação estritamente decrescente. Então a cópula para $X$ e $Y$ satisfaz

$$
C_{L}(\boldsymbol{u})=C_{L}\left(u_{1}, u_{2}\right)=\max \left\{0, u_{1}+u_{2}-1\right\}
$$

e chamamos $C_{L}$ de Limite inferior de Fréchet.

c) Finalmente, suponha que $X$ e $Y$ são variáveis aleatórias perfeitamente positivamente dependentes. Isso ocorre se $Y=T(X)$ e $T$ é uma transformação estritamente crescente. Então a cópula para $X$ e $Y$ satisfaz

$$
C_{U}(\boldsymbol{u})=C_{U}\left(u_{1}, u_{2}\right)=\min \left\{u_{1}, u_{2}\right\}
$$

e chamamos $C_{U}$ de Limite superior de Fréchet.

Os limites citados acima também são cópulas e estabelecem o seguinte teorema cuja demonstração pode ser encontrada em Nelsen (2007).

Teorema 3.2. Seja $\boldsymbol{C}$ uma copula e $\left(u_{1}, u_{2}\right) \in[0,1]^{2}$. Então, para toda copula $C\left(u_{1}, u_{2}\right)$ vale os limites de Fréchet-Hoeffding de tal maneira que:

$$
\max \left(u_{1}+u_{2}-1,0\right) \leq C\left(u_{1}, u_{2}\right) \leq \min \left(u_{1}, u_{2}\right)
$$

A cópula $C$ de $X$ e $Y$ tem a propriedade de ser invariante por transformações estritamente crescentes das marginais $F_{X}$ e $F_{Y}$, isto é, se $T_{X}$ e $T_{Y}$ são funções estritamente crescentes, então $T_{X}(X)$ e $T_{Y}(Y)$ tem a mesma cópula que $X$ e $Y$. Esta propriedade das cópulas é útil para definir medidas de dependência. 


\subsubsection{Medidas de dependência}

As medidas de dependência para gerenciamento de riscos financeiros estão vastamente descritas na literatura Embrechts et al. (2013). Para duas variáveis aleatórias $X$ e $Y$, Embrechts et al. (2001), listaram quatro propriedades desejáveis de uma medida de dependência $\delta(X, Y)$ :

1. $\delta(X, Y)=\delta(Y, X)$;

2. $-1 \leq \delta(X, Y) \leq 1$

3. $\delta(X, Y)=1$ se $X$ e $Y$ são perfeitamente positivamente dependentes; $\delta(X, Y)=-1$ se $X$ e $Y$ são perfeitamente negativamente dependentes;

4. se $T$ é estritamente monotônica, então:

$$
\delta(T(X), Y)=\left\{\begin{aligned}
\delta(X, Y), & \text { se } T \text { é crescente; } \\
-\delta(X, Y), & \text { se } T \text { é decrescente. }
\end{aligned}\right.
$$

Embrechts et al. (2013) argumentaram que a correlação linear de Pearson satisfaz apenas as duas primeiras propriedades e demonstraram que as medidas de correlação de Rho de Spearman e o Tau de Kendall satisfazem todas as quatros propriedades.

\section{a) Correlação linear de Pearson}

O coeficiente de correlação de Pearson de duas variáveis aleatórias $X$ e $Y$ é definido da seguinte forma.

$$
\rho(X, Y)=\frac{\operatorname{cov}(X, Y)}{\sqrt{\operatorname{var}(X) \operatorname{var}(Y)}},
$$

que mede a dependência linear entre $X$ e $Y$. Se $Y=a X+b$, então $\rho(X, Y)= \pm 1$. Se $X$ e $Y$ são independentes, então $\rho(X, Y)=0$.

Algumas observações sobre o coeficiente de correlação linear de Pearson são pertinentes:

i) $0<\operatorname{Var}(X), \operatorname{Var}(Y)<\infty$. 
ii) $\rho=0$ não implica independência, essa independência é válida somente para o caso em que $X$ e $Y$ têm distribuição conjunta normal bivariada.

iii) $\rho$ não é invariante sob as transformações não lineares estritamente crescentes.

iv) As distribuições marginais e o coeficiente de correlação linear de Pearson não determinam a distribuição conjunta, exceto se $X$ e $Y$ tem distribuição conjunta normal bivariada.

v) Dadas as distribuições marginais $F_{X}$ e $F_{Y}, \rho \in\left[\rho_{\text {mín }}, \rho_{m a ́ x}\right]$ em que pode ocorrer caso que $\rho_{\text {mín }}>-1$ e $\rho_{m a ́ x}<1$.

\section{b) Tau de Kendall e Rho de Spearman}

Suponha que as variáveis aleatórias $X$ e $Y$ representam os retorno em títulos financeiros. O caso comum é que ambas $X$ e $Y$ assumem valores grandes e pequenos juntos, enquanto o caso raro é o que $X$ assume um valor grande e $Y$ assume um valor pequeno (ou viceversa) ao mesmo tempo. O conceito de concordância é utilizado para medir este tipo de associação.

Dizemos que $(X, Y)$ são concordantes, se para dois vetores observados $\left(x_{1}, y_{1}\right)$ e $\left(x_{2}, y_{2}\right)$ de $(X, Y)$ com $x_{1}<x_{2}$ temos que $y_{1}<y_{2}$, ou seja,

Definição 3.1. $(X, Y)$ são vetores aleatórios com concordância se para dois vetores observados de $(X, Y),\left(x_{1}, y_{1}\right)$ e $\left(x_{2}, y_{2}\right)$, temos $\left(x_{1}-x_{2}\right)\left(y_{1}-y_{2}\right)>0$. Um vetor aleatório é discordante se não for concordante.

As medidas de concordância têm a propriedade de serem invariantes sobre transformações crescentes de $X$ e $Y$. Como um resultado, as medidas de concordância podem ser expressas como uma função de cópula entre $X$ e $Y$. A correlação linear de Pearson não é invariante sobre transformações crescentes de $X$ e $Y$, portanto, ela não é uma medida de concordância. Duas medidas de concordância comum são Rho de Spearman e Tau de Kendall. 


\section{Rho de Spearman}

Para um par de variáveis aleatórias $(X, Y)$ com distribuição conjunta $F$ e marginais $F_{X}$

e $F_{Y}$, a estatística rho de Spearman, $\rho_{S}$, é definida como a correlação (Pearson) entre $F_{X}(X)$ e $F_{Y}(Y)$. Ela é uma medida do rank de correlação em termos da transformação integral de $X$ e $Y$. Para uma cópula associada com $X$ e $Y$ pode ser mostrado que

$$
\rho_{S}=12 \iint_{I^{2}} C(u, v) d u d v-3
$$

\section{Tau de Kendall}

A estatística $\tau$ de Kendall para a distribuição $F$ é definida como:

$$
\tau=P\left\{\left(X_{1}-X_{2}\right)\left(Y_{1}-Y_{2}\right)>0\right\}-P\left\{\left(X_{1}-X_{2}\right)\left(Y_{1}-Y_{2}\right)<0\right\}
$$

Se $C$ é uma cópula associada a $F$ então pode ser mostrado que

$$
\tau=4 \iint_{I^{2}} C d C-1=4 \iint_{I^{2}} C(u, v) c(u, v) d u d v-1
$$

em que $c(u, v)$ é a densidade de copula.

Apesar de ambas $\tau$ e $\rho_{S}$ serem medidas de concordância, seus valores podem ser bem diferentes. Nelsen (2007) resume as relações entre $\tau$ e $\rho_{S}$ com as seguintes desigualdades

$$
\left\{\begin{array}{cll}
\frac{3 \tau-1}{2} & \leq \rho_{S} \leq \frac{1+2 \tau-\tau^{2}}{2}, & \\
\frac{\tau^{2}+2 \tau-1}{2} & \leq \rho_{S} \leq \frac{1+3 \tau}{2}, & \text { para } \tau>0
\end{array}\right.
$$

\subsection{Famílias de cópulas bivariadas}

Esta seção apresenta dois modelos de cópula bivariadas: cópula Normal (Seção 3.2.1) e cópula Clayton (Seção 3.2.2). Para cópulas multivariadas e outros exemplos de cópulas, vide 
Nelsen (2007), Hutchinson e Lai (1990) e Joe (1997).

\subsubsection{Cópula Normal}

A cópula Normal, ou cópula Gaussiana, (Fig. 3.1) descreve a estrutura de dependência induzida pela distribuição normal bivariada. Sem perda de generalidades, esta copula pode ser definida a partir da decomposição de Sklar aplicada a função de densidade da distribuição conjunta da variável aleatória $(X, Y)$ de variáveis aleatórias $N(0,1)$ com covariância $\alpha$. A cópula normal é dada por

$$
C_{\alpha}\left(u_{1}, u_{2}\right)=H_{\alpha}\left(\Phi^{-1}\left(u_{1}\right), \Phi^{-1}\left(u_{2}\right)\right), \alpha \in(-1,1),
$$

Função de distribuição de copula gaussiana, $\alpha=0.5$

Densidade de copula gaussiana, $\alpha=0.5$
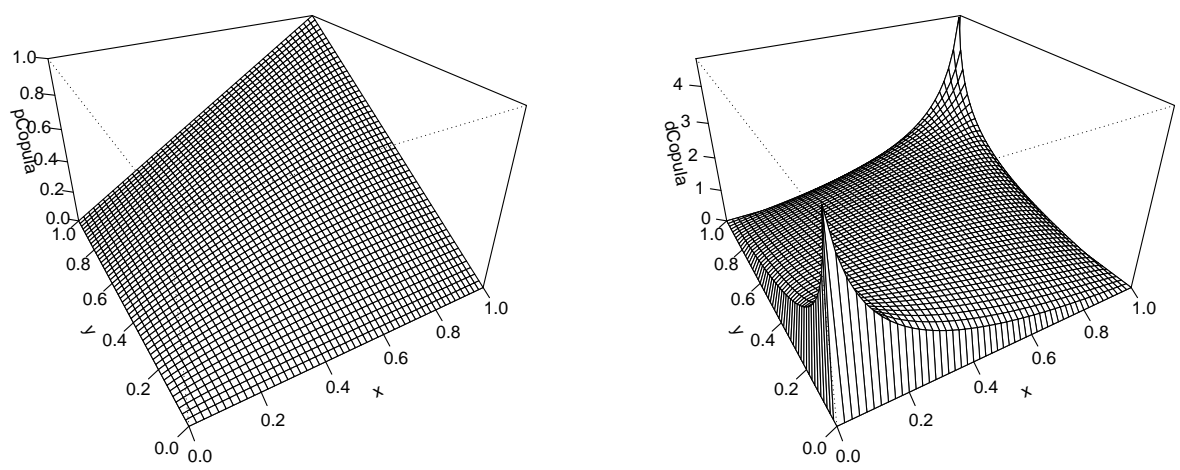

Figura 3.1: $C_{\alpha}\left(u_{1}, u_{2}\right)$

Figura 3.2: $c_{\alpha}\left(u_{1}, u_{2}\right)$

cópula Normal

Densidade de cópula Normal

onde $\Phi$ é a função de distribuição $N(0,1)$ e $H_{\alpha}$ é a função de distribuição normal padrão bivariada com covariância $\alpha$, e daí

$$
C_{\alpha}\left(u_{1}, u_{2}\right)=\frac{1}{2 \pi \sqrt{1-\alpha^{2}}} \int_{-\infty}^{\Phi^{-1}\left(u_{1}\right)} \int_{-\infty}^{\Phi^{-1}\left(u_{2}\right)} \exp \left(\frac{-\left(\xi_{1}^{2}-2 \alpha \xi_{1} \xi_{2}+\xi_{2}^{2}\right)}{2\left(1-\alpha^{2}\right)}\right) d \xi_{1} d \xi_{2}
$$


Derivando com relação a $u_{1}$ e $u_{2}$, temos a densidade de cópula é dada por

$$
c_{\alpha}\left(u_{1}, u_{2}\right)=\frac{1}{\sqrt{1-\alpha^{2}}} \exp \left(\frac{2 \alpha \xi_{1} \xi_{2}-\alpha^{2}\left(\xi_{1}^{2}+\xi_{2}^{2}\right)}{2\left(1-\alpha^{2}\right)}\right)
$$

onde $\xi_{1}=\phi^{-1}\left(u_{1}\right)$ e $\xi_{2}=\phi^{-1}\left(u_{2}\right)$.

Para a cópula normal, o tau de Kendall é dado por

$$
\tau=\frac{2}{\pi} \operatorname{arcsen}(\alpha)
$$

\subsubsection{Cópula Clayton}

A cópula Clayton (Fig. 3.3 é frequentemente atribuída a Clayton (1978), mas a sua origem remete a Kimeldori e Sampson (1975) e por isso, também é conhecida como KimeldoriSampson. É definida para $\mathbf{u} \in[0,1]^{2}$ e tem sua forma como

$$
C_{\alpha}\left(u_{1}, u_{2}\right)=\left(u_{1}^{-\alpha}+u_{2}^{-\alpha}-1\right)^{-1 / \alpha}, 0<\alpha<\infty
$$

Função de distribuição de copula Clayton, $\alpha=2$

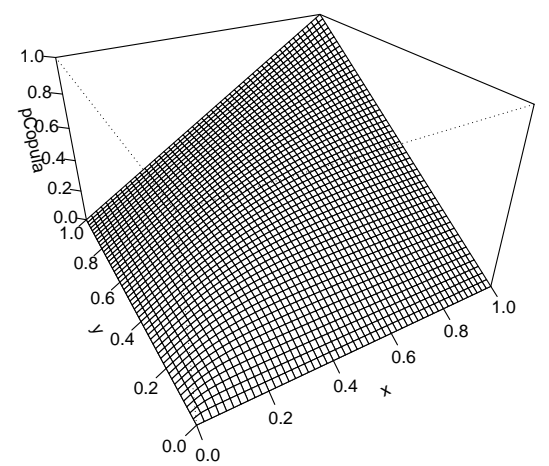

Figura 3.3: $C_{\alpha}\left(u_{1}, u_{2}\right)$ cópula Clayton
Densidade de copula Clayton, $\alpha=2$

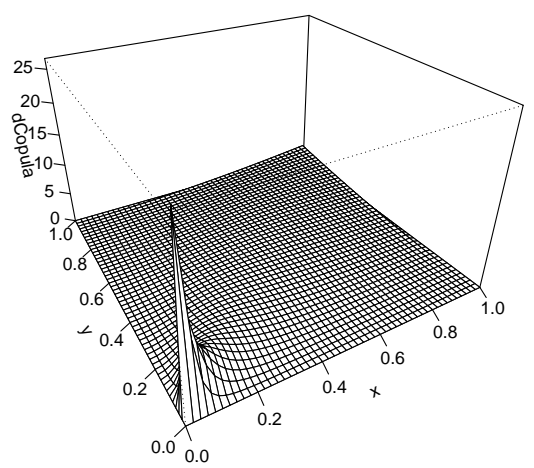

Figura 3.4: $c_{\alpha}\left(u_{1}, u_{2}\right)$

Densidade de cópula Clayton

O primeiro estudo sistemático desta família de distribuições foi publicado por Cook e 
Johnson (1981), que interpretaram o parâmetro $\alpha$ como uma medida da intensidade da dependência entre os $u_{i}, i \in\{1,2\}$. Pode-se verificar que

$$
\lim _{\alpha \rightarrow \infty} C_{\alpha}\left(u_{1}, u_{2}\right)=\min \left\{u_{1}, u_{2}\right\}=C_{U}(\mathbf{u})
$$

$C_{U}(\mathbf{u})$ é o limite superior de Frèchet de modo que a estrutura de dependência se aproxima do seu máximo quando $\alpha$ cresce para $+\infty$. Por outro lado,

$$
\lim _{\alpha \rightarrow 0} C_{\alpha}\left(u_{1}, u_{2}\right)=u_{1} u_{2}=C_{I}(\mathbf{u})
$$

portanto, a independência é obtida quando $\alpha$ tende a 0 .

A função densidade de probabilidade (Fig. 3.4 associada à cópula Clayton é dada por

$$
c_{\alpha}(\mathbf{u})=\frac{1+\alpha}{\left(u_{1} u_{2}\right)^{\alpha+1}}\left(u_{1}^{-\alpha}+u_{2}^{-\alpha}-1\right)^{-2-1 / \alpha} .
$$

Para a cópula Clayton, o tau de Kendall é dado por

$$
\tau=\frac{\alpha}{\alpha+2}
$$




\section{Capítulo 4}

\section{Cópula variante no tempo}

Até o momento apresentamos os conceitos de cópula para amostras aleatórias simples como introduzido por Sklar (1973), neste capítulo estenderemos tais definições introduzindo cópula variante no tempo para séries temporais financeiras. A literatura econométrica contém uma preponderância de evidência de que a volatilidade condicional de séries temporais financeiras muda ao longo do tempo, motivando estruturas de dependência que variam ao longo do tempo e modelos de cópula variante no tempo. Vários modelos propostos na literatura até a presente data, Patton (2002, 2004, 2006), Jondeau e Rockinger (2006), Ausin e Lopes (2010), Christoffersen, Errunza, Jacobs, e Jin (2010), Manner e Reznikova (2012) e Creal, Koopman, e Lucas (2013) consideram modelos de cópula variando no tempo em que a forma funcional da cópula é fixa e seu parâmetro é variante ao longo do tempo em função das informações defasadas, semelhante ao famoso modelo ARCH para volatilidade ( Engle (1982) e Bollerslev (1986)). Neste Capítulo, apresentamos uma abordagem alternativa semi-paramétrica para cópula variante no tempo em que o parâmetro é função do tempo.

\subsection{Motivação}

Como motivação para o uso da cópula variante no tempo, apresentamos na Figura 4.1 . duas séries financeiras: retornos das ações diárias da HP (Hewlett-Packard) e IBM (Inter- 
national Business Machines) de período 02/02/1984 a 31/12/1991.

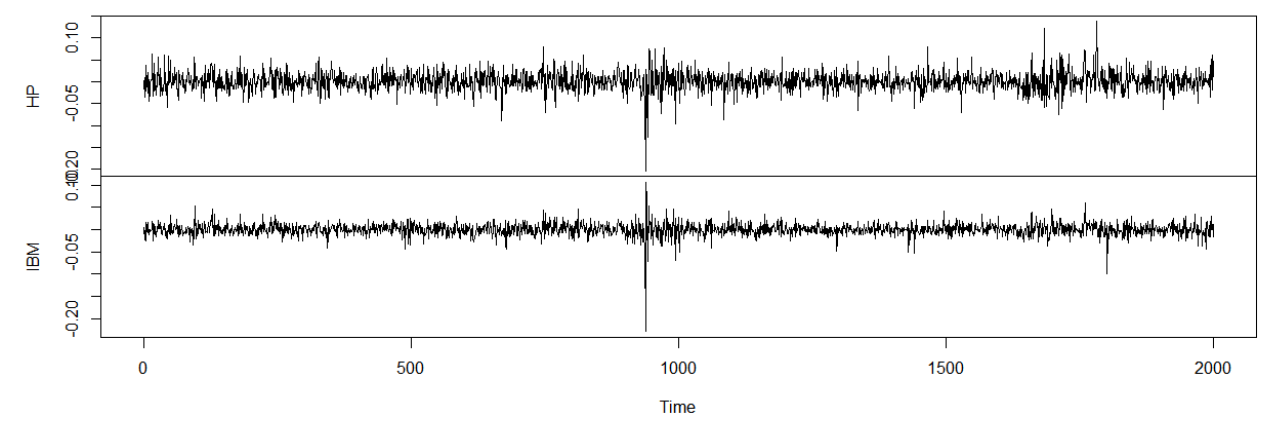

Figura 4.1: Retorno diário da HP e IBM

Essas séries de retornos, em geral, exibem pouca correlação serial, mas seus quadrados são autocorrelacionados. Em contexto multivariado, tanto a correlação cruzada quanto a volatilidade das series temporais são de grande interesse. Zivot e Wang (2007, p. 497), analisaram essas duas séries temporais de retornos e propuseram o seguinte modelo GARCH multivariado:

$$
\boldsymbol{y}_{t}=\boldsymbol{c}+\boldsymbol{\epsilon}_{t}
$$

em que $\boldsymbol{y}_{t}=\left(y_{1 t}, y_{2 t}\right)^{\top}, y_{1 t}$ : Série de retornos HP, $y_{2 t}$ : Série de retornos IBM, $\boldsymbol{c}=\left(c_{1}, c_{2}\right)^{\top}$ vetor de médias, $\boldsymbol{\epsilon}_{t}=\left(\epsilon_{1 t}, \epsilon_{2 t}\right)^{\top}$ vetor de ruído branco e $\boldsymbol{\epsilon}_{t} \sim N\left(\mathbf{0}, \Sigma_{t}\right)$ com

$$
\Sigma_{t}=A_{0} A_{0}^{\top}+A_{1} A_{1}^{\top} \circ \boldsymbol{\epsilon}_{t-1} \boldsymbol{\epsilon}_{t-1}^{\top}+b_{1} \Sigma_{t-1}
$$

sendo $A_{0}, A_{1}$ matrizes de diagonais inferiores e $b_{1}$ um valor escalar e o é o operador de Hadamard ${ }^{1}$ (vide Styan, 1973 , para mais detalhes). Usando a fórmula de variância condicional

\footnotetext{
${ }^{1} \mathrm{O}$ operador de Hadamard consiste na multiplicação elemento a elemento em duas matrizes.
} 
eles obtiveram os seguintes resultados:

$$
\begin{aligned}
\Sigma_{t} & =\left(\begin{array}{cc}
\sigma_{11, t} & \sigma_{12, t} \\
\sigma_{21, t} & \sigma_{22, t}
\end{array}\right), \\
A_{0} & =\left(\begin{array}{cc}
0,0099384 & 0 \\
0,0037295 & 0,0044583
\end{array}\right), \\
A_{1} & =\left(\begin{array}{cc}
0,321289 & 0 \\
0,1984259 & 0,2958904
\end{array}\right) \\
\boldsymbol{c} & =\left(\begin{array}{l}
0,00075 \\
0,00033
\end{array}\right), \\
b_{1} & =0,6968114 .
\end{aligned}
$$

Para ilustrar o comportamento de uma cópula variante no tempo, utilizamos os resultados obtidos por (Zivot e Wang, 2007, pág. 497) para simular duas séries multivariadas com comportamento similares às séries de retornos da HP e IBM. Mais precisamente, simulamos uma série temporal multivariada $\boldsymbol{y}_{t}=\left(y_{1 t}, y_{2 t}\right)^{\top}$ com 1000 elementos usando o modelo descrito pelas Equações 4.1, 4.2 e as estimativas da equação 4.3. A Figura 4.2, mostra o gráfico das séries simuladas $y_{1 t}$ e $y_{2 t}$.

$y_{1 t}$

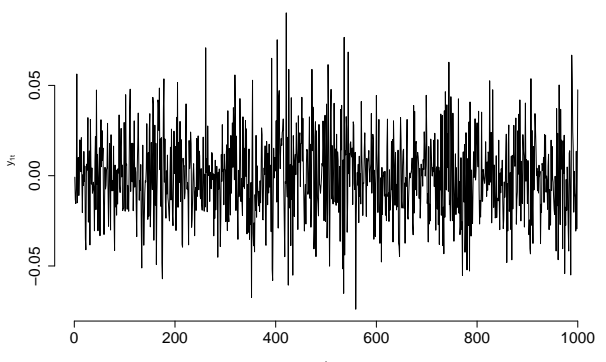

$y_{2 t}$

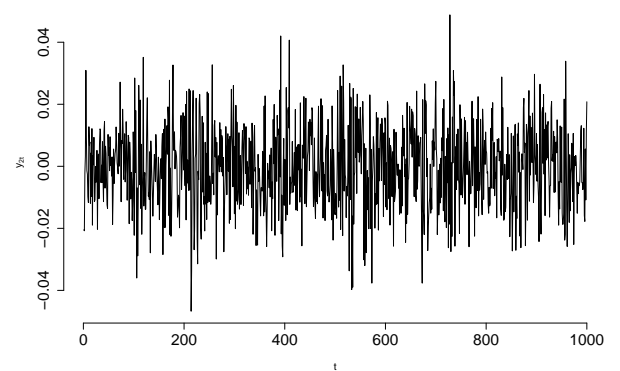

Figura 4.2: Série temporal multivariada $\boldsymbol{y}_{t}=\left(y_{1 t}, y_{2 t}\right)^{\top}$ simulada usando as estimativas da Equação 4.3 . 
Usando as propriedades de Função de Distribuição Acumulada conjunta e marginal, para cada $t$ existe uma única cópula $C_{t}$ com

$$
C_{t}(u, v)=F_{t}\left(F_{1 t}^{-1}(u), F_{2 t}^{-1}(v)\right)
$$

em que

i) $F_{t}(\cdot, \cdot)$ é a função de distribuição acumulada (f.d.a.), conjunta de $\boldsymbol{y}_{t} \mid \mathcal{F}_{t-1} \sim N\left(\boldsymbol{c} ; \Sigma_{t}\right)$, $\operatorname{com} \Sigma_{t}=\left(\begin{array}{cc}\sigma_{11, t} & \sigma_{12, t} \\ \sigma_{21, t} & \sigma_{22, t}\end{array}\right)$

ii) $F_{1 t}(\cdot)$ é a f.d.a. de $y_{1 t} \mid \mathcal{F}_{t-1} \sim N\left(\boldsymbol{c}_{1}, \sigma_{11, t}\right), F_{1 t}^{-1}(u)=\inf \left\{y_{1 t} \mid F_{1 t}\left(y_{1 t}\right) \geq u\right\}$;

iii) $F_{2 t}(\cdot)$ é a f.d.a. de $y_{2 t} \mid \mathcal{F}_{t-1} \sim N\left(\boldsymbol{c}_{2}, \sigma_{22, t}\right), \quad F_{2 t}^{-1}(v)=\inf \left\{y_{2 t} \mid F_{2 t}\left(y_{2 t}\right) \geq v\right\}$.

Na Figura 4.3. mostramos os gráficos de $C_{t}(u, u), t=2, \ldots, 1000$, para $u=0,01 ; 0,05 ; 0,25 ; 0,50$; 0,$75 ; 0,95 ; 0,99$. Note que $C_{t}(u, u)$ não apresenta um comportamento constante ao longo do tempo, para cada $u$ fixo, indicando a existência de componentes variando no tempo. 

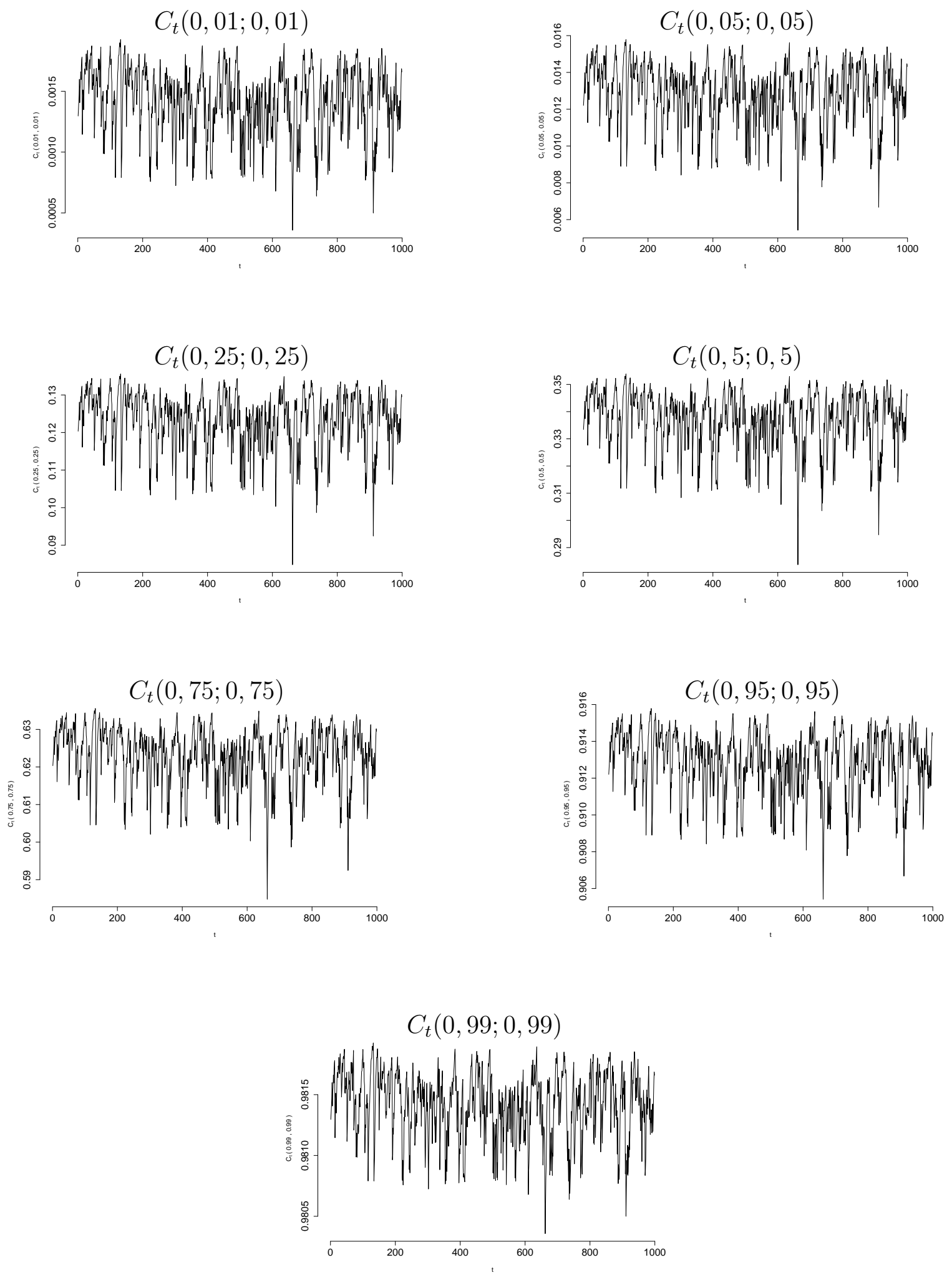

Figura 4.3: Cópula $C_{t}(u, u)$ para $u=0,01 ; 0,05 ; 0,25 ; 0,5 ; 0,75 ; 0,95 ; 0,99$. 


\subsection{Cópula variante no tempo}

Nessa seção, propomos um modelo de cópula variante no tempo para séries temporais financeiras empregando um método de estimação semi-paramétrica através de uma abordagem Bayesiana. Mais precisamente, considere uma função de cópula paramétrica $C\left(u_{1}, u_{2} \mid \theta_{t}\right)$ de séries temporais financeiras em que o parâmetro da cópula $\theta_{t}$ é uma função em t e $\boldsymbol{X}_{t}$ é uma série temporal bivariada $\boldsymbol{X}_{t}=\left(X_{1 t}, X_{2 t}\right)$ em que as distribuições marginais condicionadas nas informações passadas até o instante $t-1, X_{1 t} \mid \mathcal{F}_{t-1}$ e $X_{2 t} \mid \mathcal{F}_{t-1}$, são conhecidas. A função de distribuição acumulada conjunta de $\boldsymbol{X}_{t} \mid \mathcal{F}_{t-1}$ pode ser escrita como

$$
\begin{aligned}
F_{t}\left(x_{1 t}, x_{2 t}\right)=F\left(x_{1 t}, x_{2 t} \mid \mathcal{F}_{t-1}\right) & =C_{t}\left(F_{1 t}\left(x_{1 t}\right), F_{2 t}\left(x_{2 t}\right)\right) \\
& =C\left(F_{1 t}\left(x_{1 t}\right), F_{2 t}\left(x_{2 t}\right) \mid \theta_{t}\right),
\end{aligned}
$$

em que $C_{t}(\cdot, \cdot)$ é a função cópula condicionada em $\mathcal{F}_{t-1}$ e a função densidade conjunta de $\boldsymbol{X}_{t} \mid \mathcal{F}_{t-1}$ é dada por

$$
\begin{aligned}
f_{t}\left(x_{1 t}, x_{2 t}\right) & =c_{t}\left(F_{1 t}\left(x_{1 t}\right), F_{2 t}\left(x_{2 t}\right)\right) f_{1 t}\left(x_{1 t}\right) f_{2 t}\left(x_{2 t}\right) \\
& =c\left(F_{1 t}\left(x_{1 t}\right), F_{2 t}\left(x_{2 t}\right) \mid \theta_{t}\right) f_{1 t}\left(x_{1 t}\right) f_{2 t}\left(x_{2 t}\right),
\end{aligned}
$$

em que $c_{t}(\cdot, \cdot)$ é função densidade de cópula condicionada em $\mathcal{F}_{t-1} ; F_{1 t}(\cdot)$ e $f_{1 t}(\cdot)$ são a f.d.a. marginal e a f.d. marginal de $X_{1 t} \mid \mathcal{F}_{t-1}$, respectivamente; $F_{2 t}(\cdot)$ e $f_{2 t}(\cdot)$ são a f.d.a. marginal e a f.d. marginal de $X_{2 t} \mid \mathcal{F}_{t-1}$, respectivamente; $C\left(\cdot, \cdot \mid \theta_{t}\right)$ e $c\left(\cdot, \cdot \mid \theta_{t}\right)$ é uma cópula e sua densidade no instante $t$ para $\boldsymbol{X}_{t} \mid \mathcal{F}_{t-1}$.

Suponha que $\theta_{t} \in \Theta$, em que $\Theta$ é o espaço paramétrico. Em geral, o espaço paramétrico $\Theta$ é restrito, por exemplo, para a cópula Gaussiana, $\theta \in(-1,1)$ e para a cópula Clayton, $\theta \in(0, \infty)$. Quando $\Theta=\mathbb{R}$, o parâmetro $\theta_{t}$ pode ser estimado usando ondaletas Haar, aproximação de polinômio de Taylor e Kernel, entre outros. No entanto, geralmente o espaço $\Theta$ é um subconjunto de $\mathbb{R}$. Por essa razão, é necessário introduzir uma função de transformação 
inversível: $\Theta \rightarrow \mathbb{R}, \psi\left(\theta_{t}\right)=h(t)$ tal que $\psi^{-1}\{h(t)\}=\theta_{t}$.

Aproximamos $h(u), u \in[0,1]$ por

$$
h(u)=\sum_{k=n}^{m} a_{k} \varphi_{k}(u)
$$

em que $\varphi_{k}(u)$ pode ser ondeleta Haar, Polinômio de Taylor ou Kernel e $a_{k}$ é o coeficiente da aproximação, $k=n, n+1, \ldots, m$ em que $n, m$ são números inteiros. Denotamos $\boldsymbol{a}=\left(a_{n}, a_{n+1}, \cdots, a_{m}\right)$ o vetor dos coeficientes, que será estimado neste trabalho usando uma abordagem Bayesiana. Para isso, precisamos descrever sua função de verossimilhança, sua distribuição a priori para obter a distribuição a posteriori. O estimador pontual de $\boldsymbol{a}$ será a média da amostra simulada da distribuição a posteriori. Por definição, a função de verossimilhança de $\boldsymbol{X}_{t}$ é dada por ${ }^{2}$

$$
L\left(\boldsymbol{a} \mid x_{1}, x_{2}, \ldots, x_{T}\right)=f\left(\boldsymbol{x}_{1}, \ldots, \boldsymbol{x}_{T} \mid \boldsymbol{a}\right),
$$

em que $f$ é função densidade conjunta de $\boldsymbol{X}_{1}, \ldots, \boldsymbol{X}_{T}$. Podemos reescrever (4.4) usando condicionais

$$
\begin{aligned}
L(\boldsymbol{a}) & =f\left(\boldsymbol{x}_{T} \mid \boldsymbol{x}_{1}, \ldots, \boldsymbol{x}_{T-1}\right) \cdot f\left(\boldsymbol{x}_{T-1} \mid \boldsymbol{x}_{1}, \ldots, \boldsymbol{x}_{T-2}\right) \cdots \cdot f\left(\boldsymbol{x}_{2} \mid \boldsymbol{x}_{1}\right) \cdot f\left(\boldsymbol{x}_{1}\right) \\
& =f\left(\boldsymbol{x}_{T} \mid \mathcal{F}_{T-1}\right) \cdot f\left(\boldsymbol{x}_{T-1} \mid \mathcal{F}_{T-2}\right) \cdots, f\left(\boldsymbol{x}_{2} \mid \mathcal{F}_{1}\right) \cdot f\left(\boldsymbol{x}_{1}\right) \\
& =f\left(\boldsymbol{x}_{1}\right) \prod_{t=2}^{T} f_{t}\left(\boldsymbol{x}_{t}\right) \\
& =f\left(\boldsymbol{x}_{1}\right) \prod_{t=2}^{T} c\left(F_{1 t}\left(x_{1 t}\right), F_{2 t}\left(x_{2 t}\right) \mid \theta_{t}\right) f_{1 t}\left(x_{1 t}\right) f_{2 t}\left(x_{2 t}\right)
\end{aligned}
$$

em que $f\left(\boldsymbol{x}_{1}\right)$ é a função densidade conjunta de $\boldsymbol{x}_{1}$ e $\theta_{t}=\psi^{-1}\left(\sum_{k=n}^{m} \alpha_{k} \varphi_{k}\left(\frac{t-1}{T}\right)\right)$. Note que $f_{1 t}\left(x_{1 t}\right), f_{2 t}\left(x_{2 t}\right)$ e $f\left(\boldsymbol{x}_{1}\right)$ são funções constantes em relação a $\boldsymbol{a}$ e portanto podemos

\footnotetext{
${ }^{2}$ Afim de facilitar a apresentação dos cálculos sem carregar a notação, considere a seguinte abreviação: $\boldsymbol{X}_{t}=\left(X_{1 t}, X_{2 t}\right)$ e $\boldsymbol{x}_{t}=\left(x_{1 t}, x_{2 t}\right)$.
} 
simplificar 4.5 por

$$
\begin{aligned}
L(\boldsymbol{a}) & \propto \prod_{t=2}^{T} c\left(F_{1 t}\left(x_{1 t}\right), F_{2 t}\left(x_{2 t}\right) \mid \theta_{t}\right) \\
& =\prod_{t=2}^{T} c\left(F_{1 t}\left(x_{1 t}\right), F_{2 t}\left(x_{2 t}\right) \mid \psi^{-1}\left(h\left(\frac{t-1}{T}\right)\right)\right) .
\end{aligned}
$$

Supondo que a distribuição a priori $\boldsymbol{a} \sim N\left(\mathbf{0} ; \sigma^{2} I\right)$, em que $I$ é uma matriz identidade e $\sigma^{2}$ é um escalar positivo conhecido, a distribuição a posteriori é dada por

$$
\begin{aligned}
\pi\left(\boldsymbol{a} \mid x_{1}, x_{2}, \ldots, x_{T}\right) & \propto L(\boldsymbol{a}) \cdot \exp \left(-\frac{1}{2 \sigma^{2}} \boldsymbol{a}^{\top} \boldsymbol{a}\right) \\
& =\exp \left(-\frac{1}{2 \sigma^{2}} \boldsymbol{a}^{\top} \boldsymbol{a}\right) \prod_{t=2}^{T} c\left(F_{1 t}\left(x_{1 t}\right), F_{2 t}\left(x_{2 t}\right) \mid \psi^{-1}\left(h\left(\frac{t-1}{T}\right)\right)\right) .
\end{aligned}
$$

Neste trabalho, usamos um algoritmo de amostragem denominado Adaptative MCMC para gerar amostras da distribuição a posteriori de (4.7), cuja descrição está na Seção 4.3 . Além disso, usamos a cópula Clayton e a cópula Normal na Equação 4.7, conforme descrito nas Seções 4.2.1 e 4.2.2. Nos dois casos $\theta_{t}$ é aproximado por

a) Taylor : $h\left(\frac{t-1}{T}\right)=\psi^{-1}\left(\sum_{k=0}^{n} a_{k}\left(\frac{t-1}{T}\right)^{k}\right), t=1, \ldots, T$, em que $\boldsymbol{a}=\left(a_{0}, \ldots, a_{n}\right)^{\top}$;

b) Kernel $: h\left(\frac{t-1}{T}\right)=\psi^{-1}\left(\frac{\sum_{k=1}^{n} K_{\lambda}\left(\frac{t-1}{T}, \frac{t_{k}-1}{T}\right) a_{k}}{\sum_{k=1}^{n} K_{\lambda}\left(\frac{t-1}{T}, \frac{t_{k}-1}{T}\right)}\right), t=1, \ldots, T$, em que $K_{\lambda}\left(\frac{t-1}{T}, \frac{t_{k}-1}{T}\right)=D\left(\frac{\left|\frac{t-1}{T}-\frac{t_{k}-1}{T}\right|}{\lambda}\right)$ é o kernel de Epanechnikov $\operatorname{com} D(u)= \begin{cases}0.75\left(1-u^{2}\right), & \text { se }-1 \leq u \leq 1, \\ 0, & \text { caso contrário. }\end{cases}$

$\frac{t_{1}-1}{T}, \ldots, \frac{t_{n}-1}{T}$ são pontos igualmente espaçados em $[0,1] \operatorname{com} \frac{t_{0}-1}{T}=0$ e $\frac{t_{n}-1}{T} \approx 1 \mathrm{e}$ $\boldsymbol{a}=\left(a_{1}, \ldots, a_{n}\right)^{\top}$

c) Haar : $h\left(\frac{t-1}{T}\right)=\psi^{-1}\left(\sum_{k=0}^{2^{J}-1} a_{k} \phi_{J, k}\left(\frac{t-1}{T}\right)\right), t=1, \ldots, T$, em que $\boldsymbol{a}=\left(a_{0}, \ldots, a_{2^{J}-1}\right)^{\top}$ 
e

$\phi_{J, k}(t)=2^{\frac{J}{2}} \phi\left(2^{J} t-k\right) \operatorname{com} \phi(u)= \begin{cases}1, & \text { se } 0 \leq u<1, \\ 0, & \text { caso contrário; }\end{cases}$

d) Constante : $\theta_{t}=\theta, t=1, \ldots, T$.

\subsubsection{Cópula Clayton variante no tempo}

A cópula Clayton (vide Nelsen, 2013, para mais detalhes) é definida por

$$
C\left(u_{1}, u_{2} \mid \theta_{t}\right)=\left(u_{1}^{-\theta_{t}}+u_{2}^{-\theta_{t}}-1\right)^{\frac{1}{\theta_{t}}}, \quad \theta_{t}>0, u_{1} \in[0,1], u_{2} \in[0,1]
$$

para cada $t$ fixo, e a função densidade associada com a cópula Clayton é descrita por

$$
c\left(u_{1}, u_{2} \mid \theta_{t}\right)=\frac{1+\theta_{t}}{\left(u_{1} u_{2}\right)^{\theta_{t}+1}}\left(u_{1}^{-\theta_{t}}+u_{2}^{-\theta_{t}}-1\right)^{-2-\frac{1}{\theta_{t}}} .
$$

Então, temos que $\Theta=(0, \infty), \psi(u)=\log (u)$ e $\psi^{-1}(v)=\exp (v)$.

$$
\theta_{t}=\exp \left(h\left(\frac{t-1}{T}\right)\right)
$$

e a distribuição a posteriori de $\boldsymbol{a}$ se resume a

$$
\pi\left(\boldsymbol{a} \mid \boldsymbol{x}_{\mathbf{1}}, \boldsymbol{x}_{\mathbf{2}}, \ldots, \boldsymbol{x}_{\boldsymbol{T}}\right) \propto \exp \left(-\frac{1}{2 \sigma^{2}} \boldsymbol{a}^{\top} \boldsymbol{a}\right) \prod_{t=2}^{T} \frac{1+\theta_{t}}{\left(u_{1 t} u_{2 t}\right)^{\theta_{t}}}\left(u_{1 t}^{-\theta_{t}}+u_{2 t}^{-\theta_{t}}-1\right)^{-2-\frac{1}{\theta_{t}}}
$$

em que $u_{1 t}=F_{1 t}\left(x_{1 t}\right)$ e $u_{2 t}=F_{2 t}\left(x_{2 t}\right)$. 


\subsubsection{Cópula Normal variante no tempo}

A cópula normal (vide Denuit et al., 2006, para mais detalhes) é definida por

$$
C\left(u_{1}, u_{2} \mid \theta_{t}\right)=\frac{1}{2 \pi \sqrt{1-\theta_{t}^{2}}} \int_{-\infty}^{\Phi^{-1}\left(u_{1}\right)} \int_{-\infty}^{\Phi^{-1}\left(u_{2}\right)} \exp \left(-\frac{\delta_{1}^{2} \theta_{t}^{2}-2 \delta_{1} \delta_{2} \theta_{t}+\delta_{2}^{2} \theta_{t}^{2}}{2\left(1-\theta_{t}^{2}\right)}\right) d \xi_{1} d \xi_{2}
$$

em que $\theta_{t} \in(-1,1)$ para cada $t$ fixo, $u_{1}, u_{2} \in[0,1]$, e a função densidade associada com a cópula normal é descrita por

$$
c\left(u_{1}, u_{2} \mid \theta_{t}\right)=\frac{1}{\sqrt{1-\theta_{t}^{2}}} \exp \left(-\frac{\delta_{1}^{2} \theta_{t}^{2}-2 \delta_{1} \delta_{2} \theta_{t}+\delta_{2}^{2} \theta_{t}^{2}}{2\left(1-\theta_{t}^{2}\right)}\right)
$$

em que $\delta_{1}=\Phi^{-1}\left(u_{1}\right)$ e $\delta_{2}=\Phi^{-1}\left(u_{2}\right)$. Então, temos que $\Theta=(-1,1), \psi(u)=\log \left(\frac{1+u}{1-u}\right)$ e $\psi^{-1}(v)=\frac{\exp (v)-1}{\exp (v)+1}$. Além disso, temos que

$$
\theta_{t}=\frac{\exp \left(h\left(\frac{t-1}{T}\right)\right)-1}{\exp \left(h\left(\frac{t-1}{T}\right)\right)+1}
$$

e a distribuição a posteriori de $\boldsymbol{a}$ se resume a

$$
\pi\left(\boldsymbol{a} \mid \boldsymbol{x}_{\mathbf{1}}, \boldsymbol{x}_{\mathbf{2}}, \ldots, \boldsymbol{x}_{\boldsymbol{T}}\right) \propto \exp \left(-\frac{1}{2 \sigma^{2}} \boldsymbol{a}^{\top} \boldsymbol{a}\right) \prod_{t=2}^{T} \frac{1}{\sqrt{1-\theta_{t}^{2}}} \exp \left(\frac{\delta_{1 t}^{2} \theta_{t}^{2}-2 \delta_{1 t} \delta_{2 t} \theta_{t}+\delta_{2 t}^{2} \theta_{t}^{2}}{2\left(1-\theta_{t}^{2}\right)}\right)
$$

em que $\delta_{1 t}=\Phi^{-1}\left(F_{1 t}\left(x_{1 t}\right)\right)$ e $\delta_{2 t}=\Phi^{-1}\left(F_{2 t}\left(x_{2 t}\right)\right)$. 


\subsection{Estimação}

Na seção 4.2 obtemos a distribuição a posteriori para o vetor de coeficientes $\boldsymbol{a}=$ $\left(a_{n}, \ldots, a_{m}\right)^{\top}$. Com o objetivo de obter uma estimativa pontual para este vetor aleatório, usamos um método de Monte de Carlo via Cadeia de Markov (MCMC). Mais precisamente, MCMC é uma estratégia para amostrar valores $\left\{\boldsymbol{X}_{i}, i=1, \ldots, n\right\}$ de uma distribuição de probabilidade complexa, geralmente distribuição multivariada não gaussiana, com função densidade (ou função de probabilidade) $\pi$ definida em $X \subset \mathbb{R}^{n}$, para a qual integral do tipo

$$
\int_{X} f(v) \pi(v) d v
$$

pode ser aproximada por

$$
\frac{1}{N} \sum_{i=1}^{N} f\left(X_{i}\right) .
$$

Ou seja, se $\boldsymbol{a}_{1}, \ldots, \boldsymbol{a}_{m}$ é uma amostra de tamanho $m$ da distribuição de probabilidade do vetor aleatório de coeficientes $\boldsymbol{a}$ obtida usando MCMC, então a esperança de $\boldsymbol{a}$ pode ser aproximada por

$$
E[\boldsymbol{a}] \approx \frac{1}{m} \sum_{i=1}^{m} \boldsymbol{a}_{i}
$$

e, então, usamos a média a posteriori como estimativa pontual do vetor de coeficientes $\boldsymbol{a}$.

Os algoritmos MCMC, tais como o Metropolis-Hastings (vide Metropolis, Rosenbluth, Rosenbluth, Teller, e Teller, 1953, Hastings, 1970, para mais detalhes), são extremamente importantes e largamente utilizados para amostrar valores e obter momentos de distribuições de probabilidade $\pi$, geralmente multivariada não gaussianas, e é crucial especificar uma família de distribuição de probabilidade $\{q(\cdot \mid x) \mid x \in X\}$, denominada distribuição proposta, tarefa que pode ser extremamente difícil (vide Brooks, Gelman, Jones, e Meng, 2011, para mais detalhes). Neste trabalho, usamos um algoritmo MCMC denominado Adaptative 
MCMC proposto inicialmente por Haario, Saksman, e Tamminen (2001) e detalhado em Roberts e Rosenthal (2009). Este algoritmo atualiza a distribuição proposta através dos parâmetros da distribuição normal multivariada a cada iteração. Mais precisamente, considere uma função densidade (ou função de probabilidade) n-dimensional $\pi$ de um vetor aleatório $\boldsymbol{a}$. Então, a distribuição empírica proposta por Roberts, Rosenthal, et al. (2001) como ótima, na k-ésima iteração, supondo que o valor da cadeia de markov na (k-1)-ésima é $x_{k-1}$, é

$$
\begin{cases}N\left(x_{k-1}, \frac{(0,1)^{2}}{n} I_{n}\right), & \text { se } k \leq 2 n \\ (1-\beta) N\left(x_{k-1}, \frac{2,38^{2}}{n} \Sigma_{k}\right)+\beta N\left(x_{k-1}, \frac{(0,1)^{2}}{n} I_{n}\right), & \text { se } k>2 n\end{cases}
$$

em que $\beta$ é constante positiva pequena e Roberts e Rosenthal (2009) sugere o valor $\beta=0,05$ e $\Sigma_{k}$ é a estimativa empírica da matriz de covariância baseados nos valores $x_{1}, \ldots, x_{k-1}$. Neste trabalho, usamos a implementação do algoritmo Adaptative MCMC presente no pacote SamplerCompare implementado por Thompson (2011) no R (R Core Team, 2015). 


\section{Capítulo 5}

\section{Estudo de simulações e aplicação}

Neste capítulo, vamos comparar os métodos de cópula variante no tempo apresentados no Capítulo 4 e adicionalmente o modelo de cópula em que o parâmetro é constante no tempo. Além disso, aplicamos a cópula variante no tempo a um conjunto de dados reais, que consiste de duas séries temporais referentes ao retorno da taxa de câmbio do Real e retorno da taxa de câmbio do Euro precificados em Dólares Americanos. Este capítulo está organizado como segue: na Seção 5.1 apresentamos os modelos de cópula utilizados e as medidas usadas para compará-los; na Seção 5.2 apresentamos os resultados da simulação; e, finalmente, na Seção 5.3 fazemos uma aplicação a um conjunto de dados reais.

\subsection{Simulação}

Dividimos nosso estudo de simulação em dois casos: Cópula Normal e Cópula Clayton. Além disso, assumimos que

$$
h(u)=1+u+u^{2}, \quad u \in[0,1],
$$




$$
\theta_{t}=h\left(\frac{t-1}{T}\right), \quad t=1, \ldots, T
$$

Cópula Normal: para este caso, geramos $M=100$ séries temporais bivariadas $\boldsymbol{u}_{t}=$ $\left(u_{1 t}, u_{2 t}\right)^{\top}, t=1, \ldots, T=1024$, ou seja, $T=2^{9}$, em que $\boldsymbol{u}_{t}$ é um valor simulado da Cópula Normal com densidade dada

$$
c\left(u_{1 t}, u_{2 t} \mid \theta_{t}\right)=\frac{1}{\sqrt{1-\theta_{t}^{2}}} \exp \left(\frac{2 \theta_{t} \delta_{1} \delta_{2}-\theta_{t}^{2}\left(\delta_{1}^{2}+\delta_{2}^{2}\right)}{2\left(1-\theta_{t}^{2}\right)}\right),
$$

em que usamos o pacote copula do R (Kojadinovic e Yan, 2010) para simular $\boldsymbol{u}_{t}$ de $c\left(u_{1 t}, u_{2 t} \mid \theta_{t}\right)$.

Neste caso, a distribuição a posteriori se resume a

$$
\pi(\boldsymbol{a}) \propto \exp \left(-\frac{1}{2 \sigma^{2}} \boldsymbol{a}^{\top} \boldsymbol{a}\right) \prod_{t=2}^{T} c\left(u_{1 t}, u_{2 t} \mid \psi^{-1}\left(h\left(\frac{t-1}{T}\right)\right)\right)
$$

em que $\boldsymbol{a}=\left(a_{n}, \ldots, a_{m}\right)^{\top}$ e $h(u)=\sum_{k=n}^{m} a_{k} \varphi_{k}(u)$, conforme descrito na Seção 4.2.

Cópula Clayton: Para este caso, geramos $M=100$ séries temporais bivariadas $\boldsymbol{u}_{t}=$ $\left(u_{1 t}, u_{2 t}\right)^{\top}, t=1, \ldots, T=1024$, ou seja, $T=2^{9}$, em que $\boldsymbol{u}_{t}$ é um valor simulado da Cópula Clayton com densidade dada

$$
c\left(u_{1 t}, u_{2 t} \mid \theta_{t}\right)=\frac{1+\theta_{t}}{\left(u_{1} u_{2}\right)^{\theta_{t}+1}}\left(u_{1}^{-\theta_{t}}+u_{2}^{-\theta_{t}}-1\right)^{-2-\frac{1}{\theta_{t}}},
$$

em que usamos o pacote copula do R (Kojadinovic e Yan, 2010) para simular $\boldsymbol{u}_{t}$ de $c\left(u_{1 t}, u_{2 t} \mid \theta_{t}\right)$. 
Neste caso, a distribuição a posteriori se resume a

$$
\pi(\boldsymbol{a}) \propto \exp \left(-\frac{1}{2 \sigma^{2}} \boldsymbol{a}^{\top} \boldsymbol{a}\right) \prod_{t=2}^{T} c\left(u_{1 t}, u_{2 t} \mid \psi^{-1}\left(h\left(\frac{t-1}{T}\right)\right)\right)
$$

em que $\boldsymbol{a}=\left(a_{n}, \ldots, a_{m}\right)^{\top}$ e $h(u)=\sum_{k=n}^{m} a_{k} \varphi_{k}(u)$, conforme descrito na Seção 4.2.

Neste trabalho, $\varphi_{k}(u)$ pode ser ondeleta Haar, Polinômio de Taylor ou Kernel e $a_{k}$ é o coeficiente da aproximação, $k=n, n+1, \ldots, m$ para algum $n, m$ inteiro. Mais precisamente, $\theta_{t}$ é aproximado por

a) Taylor : $h\left(\frac{t-1}{T}\right)=\psi^{-1}\left(\sum_{k=0}^{n} a_{k}\left(\frac{t-1}{T}\right)^{k}\right), t=1,2, \ldots, T$, em que $\boldsymbol{a}=\left(a_{0}, \ldots, a_{n}\right)^{\top}$;

b) Kernel : $h\left(\frac{t-1}{T}\right)=\psi^{-1}\left(\frac{\sum_{k=1}^{n} K_{\lambda}\left(\frac{t-1}{T}, \frac{t_{k}-1}{T}\right) a_{k}}{\sum_{k=1}^{n} K_{\lambda}\left(\frac{t-1}{T}, \frac{t_{k}-1}{T}\right)}\right), t=1,2, \ldots, T$, em que $K_{\lambda}\left(\frac{t-1}{T}, \frac{t_{k}-1}{T}\right)=D\left(\frac{\left|\frac{t-1}{T}-\frac{t_{k}-1}{T}\right|}{\lambda}\right)$ é o kernel de Epanechnikov $\operatorname{com} D(u)= \begin{cases}0,75\left(1-u^{2}\right), & \text { se }-1 \leq u \leq 1 \\ 0, & \text { caso contrário. }\end{cases}$

$\frac{t_{1}-1}{T}, \ldots, \frac{t_{n}-1}{T}$ são pontos igualmente espaçados em $[0,1] \operatorname{com} \frac{t_{0}-1}{T}=0$ e $\frac{t_{n}-1}{T} \approx 1$ e $\boldsymbol{a}=\left(a_{1}, \ldots, a_{n}\right)^{\top}$;

c) Haar : $h\left(\frac{t-1}{T}\right)=\psi^{-1}\left(\sum_{k=0}^{2^{J}-1} a_{k} \phi_{J, k}\left(\frac{t-1}{T}\right)\right), t=1, \ldots, T$ em que $\boldsymbol{a}=\left(a_{0}, \ldots, a_{2^{J}-1}\right)^{\top}$ e $\phi_{J, k}(t)=2^{\frac{J}{2}} \phi\left(2^{J} t-k\right) \operatorname{com} J=\lfloor 0,5 \log 2(1024)\rfloor=5$ e $\phi(u)= \begin{cases}1, & \text { se } 0 \leq u<1, \\ 0, & \text { caso contrário; }\end{cases}$ d) Constante : $\theta_{t}=\theta, t=1, \ldots, T$.

Como comentado no Capítulo 4, usamos o método Adaptative $M C M C$ para gerar amostras da distribuição a posteriori $\pi(\boldsymbol{a})$ conforme descrito por Roberts e Rosenthal (2009) e implementado por Thompson (2011) no pacote SamplerCompare do R. 
Comparamos as estimativas $\hat{\theta}_{t}^{k}, k=1, \ldots, M=100, t=1, \ldots, T=1024$, medidas de viés e Erro Quadrático Médio para o parâmetro variante no tempo, $\theta_{t}$, para a medida de dependência tau de Kendall, $\tau_{t}$, e para Cópula nos quantis $\{0,01 ; 0,05 ; 0,25 ; 0,50 ; 0,75 ; 0,95 ; 0,99\}$.

Quantis da cópula: Para cada $t$ e para cada $\boldsymbol{u}=(u, u)^{\top}, u \in\{0,01 ; 0,05 ; 0,25 ; 0,50$;

$0,75 ; 0,95 ; 0,99\}$, podemos calcular o viés e o erro quadrático médio por

$$
\begin{aligned}
\operatorname{viés}_{u}(t) & =\mathrm{E}\left(C\left(u, u \mid \theta_{t}\right)-C\left(u, u \mid \hat{\theta}_{t}\right)\right) \\
& =\frac{1}{M} \sum_{k=1}^{M}\left(C\left(u, u \mid \theta_{t}\right)-C\left(u, u \mid \hat{\theta}_{t}^{k}\right)\right), \\
\operatorname{EQM}_{u}(t) & =\mathrm{E}\left(C\left(u, u \mid \theta_{t}\right)-C\left(u, u \mid \hat{\theta}_{t}\right)\right)^{2} \\
& =\frac{1}{M} \sum_{k=1}^{M}\left(C\left(u, u \mid \theta_{t}\right)-C\left(u, u \mid \hat{\theta}_{t}^{k}\right)\right)^{2} .
\end{aligned}
$$

Note que $\hat{\theta}_{t}^{k}=\psi^{-1}\left(\hat{h}\left(\frac{t-1}{T}\right)\right)$ para o caso dos modelos de cópula variante no tempo proposto no Capítulo 4 e $\theta_{t}^{k}=\hat{\theta}^{k}$ para caso constante estimado por máxima verossimilhança.

Observe que viés ${ }_{u}(t)$ e $E Q M_{u}(t)$ são funções de $t$. Para obter uma medida escalar para o viés e o erro quadrático médio (EQM) usamos integração:

$$
\begin{aligned}
\operatorname{viés}(u) & =\int_{0}^{1} \mathrm{E}\left(C\left(u, u \mid \psi^{-1}(h(\xi))\right)-C\left(u, u \mid \psi^{-1}(\hat{h}(\xi))\right)\right) d \xi \\
& =\frac{1}{1024} \sum_{t=2}^{1024} \mathrm{E}\left(C \left(u, u \mid \psi^{-1}\left(h\left(\frac{t-1}{T}\right)\right)-C\left(u, u \mid \psi^{-1}\left(\hat{h}\left(\frac{t-1}{T}\right)\right)\right),\right.\right. \\
\operatorname{EQM}(u) & =\int_{0}^{1} \mathrm{E}\left(C\left(u, u \mid \psi^{-1}(h(\xi))\right)-C\left(u, u \mid \psi^{-1}(\hat{h}(\xi))\right)\right)^{2} d \xi \\
& =\frac{1}{1024} \sum_{t=2}^{1024} \mathrm{E}\left(C \left(u, u \mid \psi^{-1}\left(h\left(\frac{t-1}{T}\right)\right)-C\left(u, u \mid \psi^{-1}\left(\hat{h}\left(\frac{t-1}{T}\right)\right)\right)^{2} .\right.\right.
\end{aligned}
$$


Parâmetro variante no tempo: Para cada $t$, podemos aproximar o viés e o erro quadrático médio (EQM) na estimativa de $\theta_{t}$ por

$$
\begin{aligned}
\operatorname{viés}_{\theta}(t) & =\mathrm{E}\left(\theta_{t}-\theta_{t}^{k}\right) \\
& =\frac{1}{M} \sum_{k=1}^{M}\left(\theta_{t}-\hat{\theta}_{t}^{k}\right), \\
\operatorname{EQM}_{\theta}(t) & =\mathrm{E}\left(\theta_{t}-\theta_{t}^{k}\right)^{2} \\
& =\frac{1}{M} \sum_{k=1}^{M}\left(\theta_{t}-\hat{\theta}_{t}^{k}\right)^{2} .
\end{aligned}
$$

Para obter uma medida escalar para o viés e para o erro quadrático médio (EQM) usamos integração:

$$
\begin{aligned}
\operatorname{viés}(\theta) & =\int_{0}^{1} \mathrm{E}\left(\psi^{-1}(h(u))-\psi^{-1}(\hat{h}(u))\right) d u \\
& =\frac{1}{1024} \sum_{k=2}^{1024} \mathrm{E}\left(\psi^{-1}\left(h\left(\frac{t-1}{T}\right)\right)-\psi^{-1}\left(\hat{h}\left(\frac{t-1}{T}\right)\right)\right), \\
\operatorname{EQM}(\theta) & =\int_{0}^{1} \mathrm{E}\left(\psi^{-1}(h(u))-\psi^{-1}(\hat{h}(u))\right)^{2} d u \\
& =\frac{1}{1024} \sum_{k=2}^{1024} \mathrm{E}\left(\psi^{-1}\left(h\left(\frac{t-1}{T}\right)\right)-\psi^{-1}\left(\hat{h}\left(\frac{t-1}{T}\right)\right)\right)^{2} .
\end{aligned}
$$


Tau de Kendall: De forma similar ao parâmetro tempo variante $\theta_{t}$, para cada $t$ podemos calcular o viés e erro quadrático médio para a medida de dependência $\tau(t)$

$$
\begin{aligned}
\operatorname{viés}_{\tau}(t) & =\mathrm{E}\left(\tau(t)-\tau^{k}(t)\right) \\
& =\frac{1}{M} \sum_{k=1}^{M}\left(\tau(t)-\hat{\tau}^{k}(t)\right), \\
E Q M_{\tau}(t) & =\mathrm{E}\left(\tau(t)-\tau^{k}(t)\right)^{2} \\
& =\frac{1}{M} \sum_{k=1}^{M}\left(\tau(t)-\hat{\tau}^{k}(t)\right)^{2} .
\end{aligned}
$$

Para obter uma medida escalar para o viés e para o erro quadrático médio, usamos integração

$$
\begin{aligned}
\operatorname{viés}(\tau) & =\int_{0}^{1} \mathrm{E}\left(\tau\left(\psi^{-1}(h(u))\right)-\tau\left(\psi^{-1}(\hat{h}(u))\right)\right) d u \\
& =\frac{1}{1024} \sum_{t=2}^{1024} \mathrm{E}\left(\tau\left(\psi^{-1}\left(h\left(\frac{t-1}{T}\right)\right)\right)-\tau\left(\psi^{-1}\left(\hat{h}\left(\frac{t-1}{T}\right)\right)\right)\right), \\
\operatorname{EQM}(\tau) & =\int_{0}^{1} \mathrm{E}\left(\tau\left(\psi^{-1}(h(u))\right)-\tau\left(\psi^{-1}(\hat{h}(u))\right)\right)^{2} d u \\
& =\frac{1}{1024} \sum_{t=2}^{1024} \mathrm{E}\left(\tau\left(\psi^{-1}\left(h\left(\frac{t-1}{T}\right)\right)\right)-\tau\left(\psi^{-1}\left(\hat{h}\left(\frac{t-1}{T}\right)\right)\right)\right)^{2} .
\end{aligned}
$$

\subsection{Resultados do estudo de simulação}

Dividimos a análise dos resultados da simulação em duas partes: caso Clayton e caso Normal. Em ambos os casos analisamos a cópula constante, com o parâmetro fixo no tempo e a cópula variante no tempo, onde $\theta$ tem seu comportamento descrito por uma função, que pode ser estimada pelos métodos de aproximação de funções: Ondaletas Haar, polinômios de Taylor e Kernel smoother com núcleo Epanechnikov. Estes métodos serão tratados apenas por: Constante, Haar, Taylor e Kernel, respectivamente.

A análise descritiva da eficiência dos métodos utilizados foi feita através do viés e do EQM. Para facilitar a análise, convencionamos tratar a abordagem semi paramétrica para $\theta_{t}$, 
ou seja, $\theta$ variante no tempo, simplesmente por abordagem variante no tempo e a abordagem usual, em que o parâmetro é considerado constante ao longo do tempo, por abordagem Constante.

\subsubsection{Caso Clayton}

A análise dos parâmetros estimados, para a cópula Clayton, está na seguinte ordem: Ajuste da cópula, $\theta_{t}$ e $\tau$ de Kendall.

Tabela 5.1: viés $(\boldsymbol{u})$ para cópula Clayton

\begin{tabular}{lrrrrrrr}
\hline viés $(\boldsymbol{u}) \times 10^{-3}$ & 0,01 & 0,05 & 0,25 & 0,50 & 0,75 & 0,95 & 0,99 \\
\hline Constante & $-0,0080$ & $-0,0399$ & $-0,0845$ & 1,2325 & 4,4298 & 1,8445 & 0,1541 \\
Haar & 0,1083 & 0,5335 & 2,3765 & 3,5406 & 2,6905 & 0,3567 & 0,0205 \\
Taylor & $\mathbf{0 , 0 0 5 0}$ & $\mathbf{0 , 0 2 4 9}$ & $\mathbf{0 , 1 1 6 9}$ & $\mathbf{0 , 1 8 8 4}$ & $\mathbf{0 , 1 6 7 2}$ & $\mathbf{0 , 0 3 4 6}$ & $\mathbf{0 , 0 0 2 6}$ \\
Kernel & 0,0700 & 0,3498 & 1,6986 & 2,9576 & 2,8832 & 0,6028 & 0,0445 \\
\hline
\end{tabular}

A partir da Tabela 5.1 pode-se perceber que, de acordo com o viés, existem indícios de subestimação da cópula pela abordagem variante no tempo. Por outro lado, a abordagem Constante superestima nos quantis inferiores e passa a subestima-la nos quantis superiores. Em ambas as abordagens os quantis centrais apresentam valores maiores de viés do que nos quantis extremos.

Comparando a abordagem variante no tempo com a abordagem Constante, nos quantís inferiores a abordagem Constante apresenta viés menor que Haar e Kernel. No entanto, não apresenta resultados melhores que Taylor nestes quantis, com exceção do quantil 0,25.

Tabela 5.2: $E Q M(\boldsymbol{u})$ para cópula Clayton

\begin{tabular}{lrrrrrrr}
\hline$E Q M(\boldsymbol{u}) \times 10^{-3}$ & 0,01 & 0,05 & 0,25 & 0,50 & 0,75 & 0,95 & 0,99 \\
\hline Constante & 0,00034 & 0,00844 & 0,20597 & 0,67986 & 0,70588 & 0,03196 & 0,00017 \\
Haar & 0,00026 & 0,00608 & 0,10776 & 0,22478 & 0,13375 & 0,00353 & 0,00002 \\
Taylor & $\mathbf{0 , 0 0 0 0 1}$ & $\mathbf{0 , 0 0 0 1 5}$ & $\mathbf{0 , 0 0 3 3 8}$ & $\mathbf{0 , 0 0 9 3 4}$ & $\mathbf{0 , 0 0 8 0 4}$ & $\mathbf{0 , 0 0 0 4 8}$ & $<\mathbf{0 , 0 0 0 0 1}$ \\
Kernel & 0,00002 & 0,00057 & 0,01318 & 0,03616 & 0,02910 & 0,00124 & 0,00001 \\
\hline
\end{tabular}

A partir da Tabela 5.2 verifica-se que o erro quadrático médio da abordagem variante no tempo por Haar, Taylor e Kernel foram menores em todos os quantis em relação à abordagem 
Constante. Entre os três métodos de estimação variantes no tempo o método que apresentou os menores EQM foi o Taylor, seguido pelo Kernel e Haar. É possível notar ainda que o EQM é menor nas extremidades em todos os casos.

Assim, observando as evidências nas tabelas, verifica-se que mesmo quando o viés da abordagem variante no tempo é maior comparando com a Constante, aquela possui um erro quadrático médio menor. Isto nos leva a crer que a abordagem variante no tempo apresenta um erro menor ao estimar a cópula.

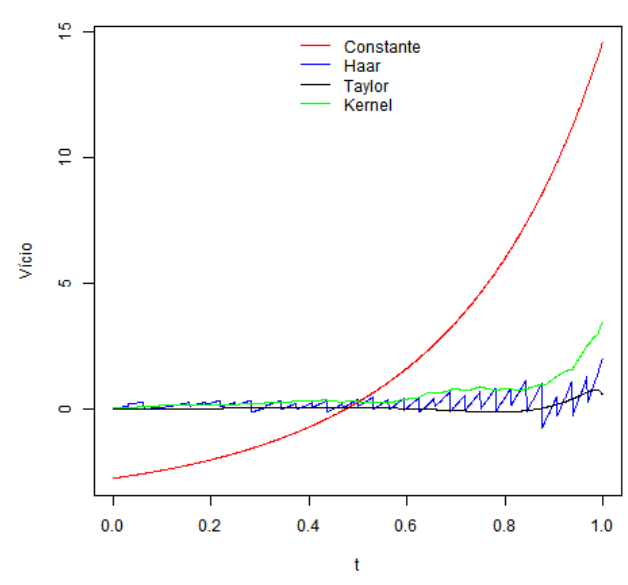

(a) viés $s_{\theta}(t)$

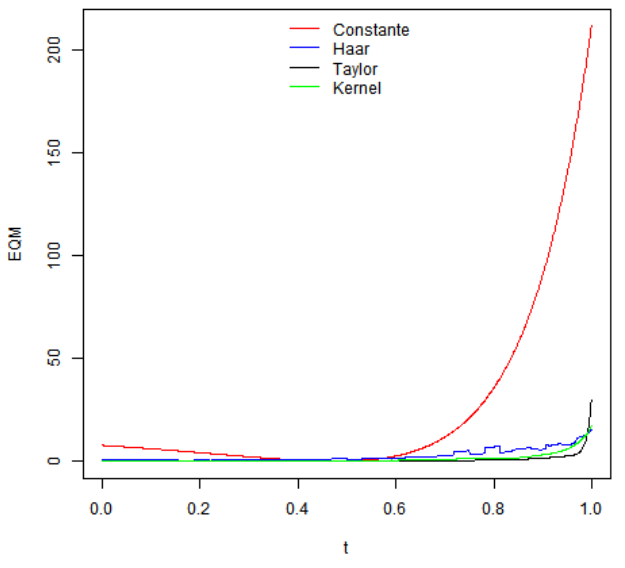

(b) $E Q M_{\theta}(t)$

Figura 5.1: viés $s_{\theta}(t)$ e $E Q M_{\theta}(t)$ Caso Clayton

A Figura 5.1a mostra o viés $s_{\theta}(t)$, é possível notar que o viés do estimador para o parâmetro $\theta$, quando considerado constante ao longo do tempo (curva vermelha), começa superestimando e passa a subestimar o parâmetro ao longo da série conforme o seu viés vai aumentando gradativamente. Na abordagem variante no tempo, o viés se mantém em torno de zero e Taylor apresenta o melhor ajuste.

A Figura 5.1b, mostra o $E Q M_{\theta}(t)$, notamos indícios de que o erro quadrático médio nas duas abordagens são equivalentes. No entanto, ao longo da série, o erro quadrático médio, quando $\theta$ é constante ao longo do tempo, apresenta um aumento na variabilidade para as estimativas, já a abordagem variante no tempo não apresenta esse problema.

As Tabelas 5.3a e 5.3b mostram o viés e o erro quadrático médio do estimador de $\theta$ 
Tabela 5.3: viés $(\theta)$ e $\operatorname{EQM}(\theta)$ Cópula Clayton

(a) $\operatorname{viés}(\theta)$

\begin{tabular}{lr}
\hline Constante & 1,9446 \\
Haar & 0,2270 \\
Taylor & $\mathbf{0 , 0 3 1 9}$ \\
kernel & 0,5488 \\
\hline
\end{tabular}

(b) $\operatorname{EQM}(\theta)$

\begin{tabular}{lr}
\hline Constante & 24,9133 \\
Haar & 2,7448 \\
Taylor & $\mathbf{0 , 6 9 6 5}$ \\
kernel & 1,2700 \\
\hline
\end{tabular}

respectivamente e verifica-se que quando considerada a abordagem variante no tempo, ambas as medidas são menores do que na abordagem em que $\theta$ é considerado constante.

Notamos portanto, que quando considerada a abordagem variante no tempo, o aumento de informação melhora a acurácia do estimador do parâmetro. Nota-se ainda que a aproximação da função por Taylor apresenta os melhores resultados. Já na abordagem Constante há indícios de que a variabilidade do estimador cresce ao longo da série.

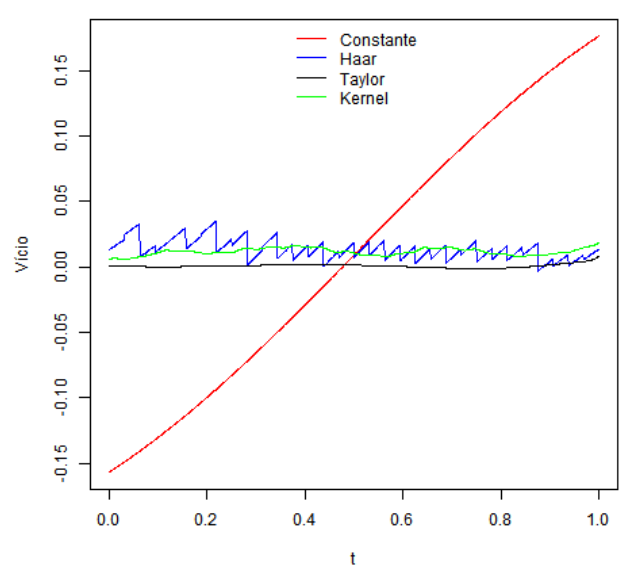

(a) $v i \mathrm{e} s_{\tau}(t)$

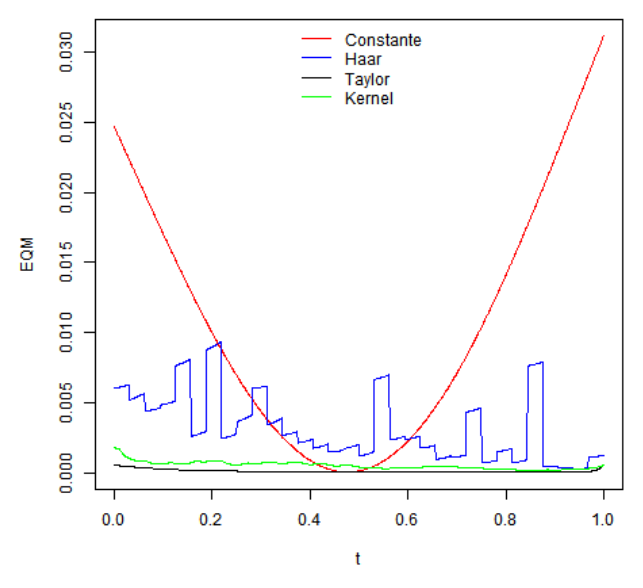

(b) $E Q M_{\tau}(t)$

Figura 5.2: viés $s_{\tau}(t)$ e $E Q M_{\tau}(t)$ Caso Clayton

Na Figura $5.2 \mathrm{a}$ temos o viés $s_{\tau}(t)$, verifica-se que o viés do estimador, para a medida $\tau$ de Kendall, permanece com variação pequena em torno do zero para a abordagem variante no tempo. Já na abordagem Constante, no início da série, o modelo está superestimando o $\tau$ de Kendall e passa a subestima-lo ao longo da série. Podemos notar ainda que a aproximação por Taylor apresenta melhores resultados do que Haar e Kernel.

$\mathrm{Na}$ Figura $5.2 \mathrm{~b}$ temos $E Q M_{\tau}(t)$, verifica-se que o EQM, na abordagem variante no tempo, 
para o estimador de $\tau$ assume valores próximos de zero ao longo da série. Enquanto que para a abordagem em que $\theta$ é constante, o estimador assume erro quadrático médio maior no início e no final da série.

Tabela 5.4: viés $(\tau)$ e $\operatorname{EQM}(\tau)$ cópula Calyton

\begin{tabular}{lr}
\multicolumn{2}{c}{$($ a) viés $(\tau)$} \\
\hline Constante & 0,0093 \\
Haar & 0,0132 \\
Taylor & $\mathbf{0 , 0 0 0 7}$ \\
Kernel & 0,0116 \\
\hline
\end{tabular}

(b) $\operatorname{EQM}(\tau)$

\begin{tabular}{lr}
\hline Constante & 0,0105 \\
Haar & 0,0032 \\
Taylor & $\mathbf{0 , 0 0 0 1}$ \\
Kernel & 0,0005 \\
\hline
\end{tabular}

A média dos vieses exposta na Tabela 5.4a para a abordagem Constante é menor do que a média dos vieses estimados por Haar e Kernel. Entretanto, quando analisamos o erro quadrático médio, na Tabela 5.4b percebemos que tanto Haar quanto Kernel apresentam um EQM menor favorecendo a escolha da abordagem variante no tempo. Nota-se ainda, que nesta abordagem, Taylor é o que apresenta o melhor ajuste.

Ambas as abordagens estimam bem a medida $\tau$. No entanto, a abordagem variante no tempo tem a vantagem de apresentar variabilidade menor nas estimativas.

Após verificar as estimativas para a cópula, para $\theta$ e para o $\tau$, concluímos que a abordagem considerando o parâmetro $\theta_{t}$, ou seja, variante no tempo, apresenta melhores ajustes, em detrimento da abordagem que considera o parâmetro $\theta$ constante. Notamos ainda que Taylor apresenta os melhores ajustes em todos os casos.

\subsubsection{Caso Normal}

A análise dos parâmetros estimados, para a cópula Normal, estão na seguinte ordem: Ajuste da cópula, $\theta_{t}$ e $\tau$ de Kendall.

Analisando a Tabela 5.5 vemos que a abordagem Constante e a abordagem variante no tempo subestimam a cópula. Pode-se perceber ainda que a abordagem Constante é mais eficiente nos quantis centrais do que a abordagem variante no tempo nos casos Haar e Kernel. No entanto, Taylor é mais eficiente em todos os quantis em ambas as abordagens. 
Tabela 5.5: viés $(\boldsymbol{u})$ cópula Normal

\begin{tabular}{lrrrrrrr}
\hline viés $(\boldsymbol{u}) \times 10^{-3}$ & 0,01 & 0,05 & 0,25 & 0,5 & 0,75 & 0,95 & 0,99 \\
\hline Constante & 0,2852 & 1,0029 & 2,5269 & 2,9730 & 2,5269 & 1,0029 & 0,2852 \\
Haar & 0,0760 & 0,4998 & 2,4019 & 3,3012 & 2,4019 & 0,4998 & 0,0760 \\
Taylor & $\mathbf{0 , 0 2 2 9}$ & $\mathbf{0 , 1 1 2 2}$ & $\mathbf{0 , 4 2 3 2}$ & $\mathbf{0 , 5 5 3 9}$ & $\mathbf{0 , 4 2 3 2}$ & $\mathbf{0 , 1 1 2 2}$ & $\mathbf{0 , 0 2 2 9}$ \\
kernel & 0,2009 & 0,9944 & 3,8274 & 5,0368 & 3,8274 & 0,9944 & 0,2009 \\
\hline
\end{tabular}

Tabela 5.6: $\operatorname{EQM(u)~cópula~Normal~}$

\begin{tabular}{lrrrrrrr}
\hline$E Q M(\boldsymbol{u}) \times 10^{-3}$ & 0,01 & 0,05 & 0,25 & 0,50 & 0,75 & 0,95 & 0,99 \\
\hline Constante & 0,0018 & 0,0420 & 0,5759 & 0,9805 & 0,5759 & 0,0420 & 0,0018 \\
Haar & 0,0004 & 0,0109 & 0,1802 & 0,3209 & 0,1802 & 0,0109 & 0,0004 \\
Taylor & $\mathbf{0 , 0 0 0 1}$ & $\mathbf{0 , 0 0 1 2}$ & $\mathbf{0 , 0 1 6 6}$ & $\mathbf{0 , 0 2 8 7}$ & $\mathbf{0 , 0 1 6 6}$ & $\mathbf{0 , 0 0 1 2}$ & $\mathbf{0 , 0 0 0 1}$ \\
Kernel & 0,0001 & 0,0033 & 0,0522 & 0,0917 & 0,0522 & 0,0033 & 0,0001 \\
\hline
\end{tabular}

Analisando a Tabela 5.6, referente ao erro quadrático médio do estimador da cópula, percebemos que em todos os quantis a abordagem variante no tempo apresenta valores menores que a abordagem Constante.

Com base nas análise do viés e do EQM temos indícios para acreditar que a abordagem variante no tempo estima melhor a cópula do que a abordagem Constante.

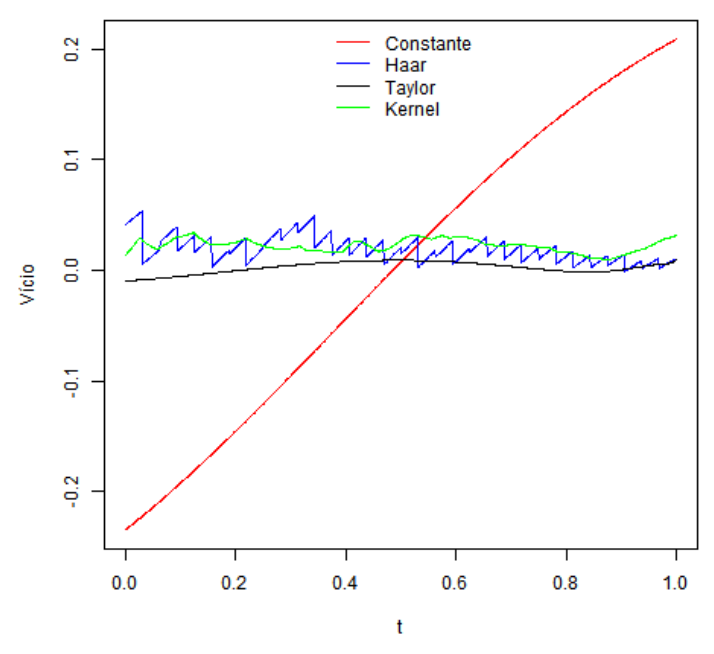

(a) $v i e ́ s s_{\theta}(t)$

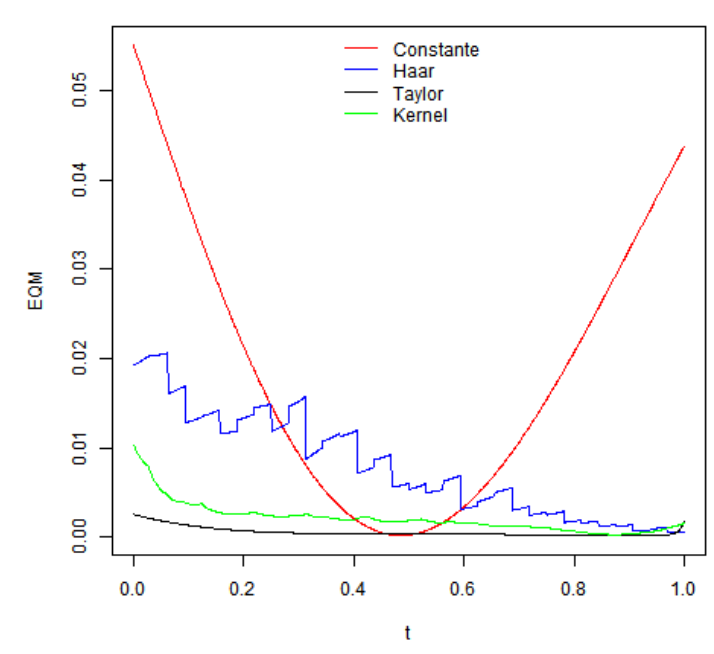

(b) $E Q M_{\theta}(t)$

Figura 5.3: viés $s_{\theta}(t)$ e $E Q M_{\theta}(t)$ Caso Normal

Na Figura 5.3a temos o viés $s_{\theta}(t)$. É possível notar que os vieses dos estimadores para o 
parâmetro $\theta$, estimado pela abordagem variante no tempo tem variação pequena em torno do zero. Enquanto a abordagem Constante (curva vermelha), inicialmente, superestima o parâmetro e passa a subestimá-lo ao longo da série conforme seu viés aumenta gradativamente.

Na Figura $5.3 \mathrm{~b}$ temos o $E Q M_{\theta}(t)$. Pode-se observar, indícios de, que ao longo da série, o erro quadrático médio da abordagem variante no tempo vai diminuindo, mantendo-se próximo de zero. Já a abordagem Constante tem valores altos de erro quadrático médio no início e no final da série, quando comparados à abordagem variante no tempo.

Tabela 5.7: viés $(\theta)$ e $E Q M(\theta)$ cópula Normal

(a) viés $(\theta)$ Normal

\begin{tabular}{lr}
\hline Constante & $-0,0001$ \\
Haar & 0,0188 \\
Taylor & 0,0025 \\
Kernel & 0,0225 \\
\hline
\end{tabular}

(b) $\operatorname{EQM}(\theta)$ Normal

\begin{tabular}{lr}
\hline Constante & 0,0184 \\
Haar & 0,0079 \\
Taylor & $\mathbf{0 , 0 0 0 6}$ \\
Kernel & 0,0021 \\
\hline
\end{tabular}

Nas Tabelas 5.7a e 5.7b estão o viés $(\theta)$ e o $E Q M(\theta)$, respectivamente. Verifica-se que o viés na abordagem Constante é menor que na abordagem variante no tempo. No entanto, o erro quadrático médio do estimador de $\theta$ quando é considerado a abordagem variante no tempo é melhor.

Notamos novamente que na abordagem variante no tempo o aumento de informação melhora e mantém a acurácia dos estimadores do parâmetro, com destaque para Taylor que apresenta o menor EQM. Na abordagem Constante, o aumento do tamanho da série parece gerar uma variabilidade maior.

A Figura $5.4 \mathrm{a}$ mostra o vié $s_{\tau}(t)$, nela verifica-se que o viés do estimador para a medida $\tau$ de Kendall permanece com variação pequena em torno do zero para a abordagem variante no tempo e, dentro dessa abordagem, Taylor apresenta o menor viés ao longo da série. Já a abordagem Constante superestima o $\tau$ de Kendall no início da série e passa a subestimá-lo ao longo dela.

A Figura $5.4 \mathrm{~b}$ mostra o $E Q M_{\tau}(t)$. Verifica-se pela Figura que, na abordagem variante 


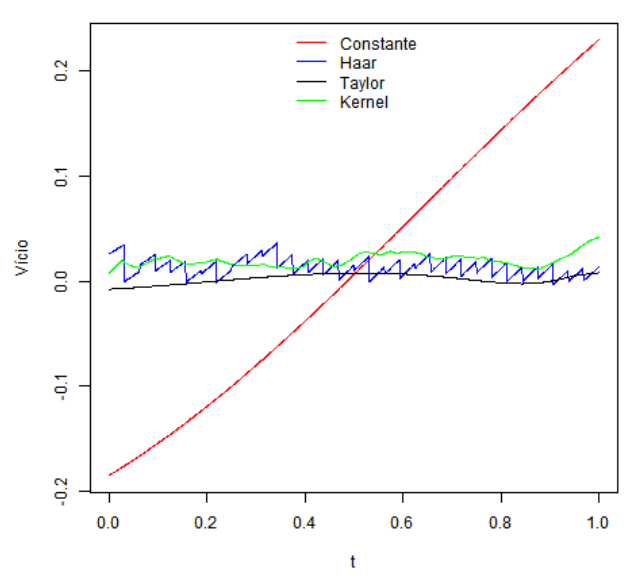

(a) $v i e ́ s_{\tau}(t)$

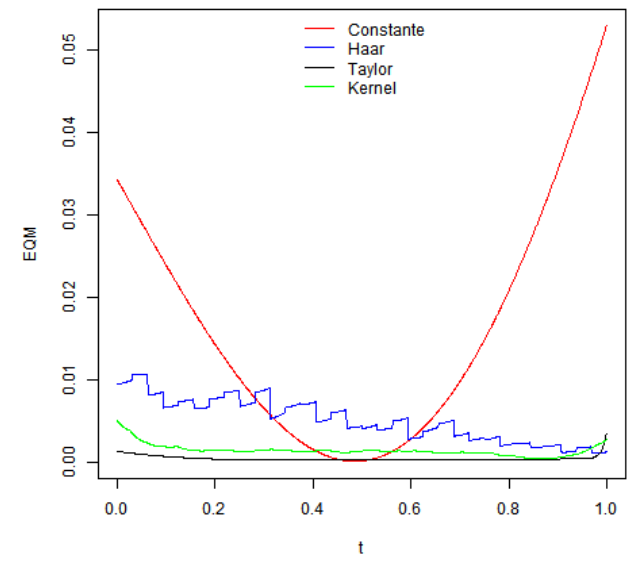

(b) $E Q M_{\tau}(t)$

Figura 5.4: viés $s_{\tau}(t)$ e $E Q M_{\tau}(t)$ Caso Normal

no tempo, o EQM para o estimador de $\tau$ assume valores próximos de zero ao longo da série. Enquanto que para a abordagem Constante o erro quadrático médio é maior no início e no final da série.

Tabela 5.8: viés $(\tau)$ e $E Q M(\tau)$ cópula Normal

\begin{tabular}{|c|c|c|c|}
\hline (a) viés $(\tau)$ & Normal & (b) $E Q M$ & Normal \\
\hline Constante & 0,0119 & Constante & 0,0157 \\
\hline Haar & 0,0132 & Haar & 0,0051 \\
\hline Taylor & 0,0022 & Taylor & 0,0005 \\
\hline Kernel & 0,0201 & Kernel & 0,0015 \\
\hline
\end{tabular}

O viés, exposto na Tabela $5.8 \mathrm{a}$ para a abordagem Constante é menor. Entretanto, quando analisamos o erro quadrático médio, na Tabela 5.8b, a abordagem variante no tempo apresenta um valor menor que a abordagem Constante o que favorece a escolha da abordagem variante no tempo.

Ambas as abordagens estimam bem a medida $\tau$. No entanto, a abordagem variante no tempo tem a vantagem de apresentar variabilidade menor nas estimativas. Percebemos ainda, que Taylor apresenta o menor viés e o menor EQM.

Após verificar as estimativas para a cópula, para $\theta$ e para o $\tau$, concluímos que a abordagem variante no tempo, utilizando a cópula normal, também apresenta melhores resultados 
que a abordagem Constante. Percebemos também que Taylor apresentou o melhor ajuste tanto no caso normal quanto no caso Clayton.

\subsection{Aplicação}

Para ilustrar a metodologia, usamos o retorno, $r_{t}=\ln \left(\frac{P_{t}}{P_{t-1}}\right)$, das taxas de câmbio do Euro e do Real precificadas em dólar, Figura 5.5. compreendido no período de 05/10/2006 até $04 / 08 / 2016$.
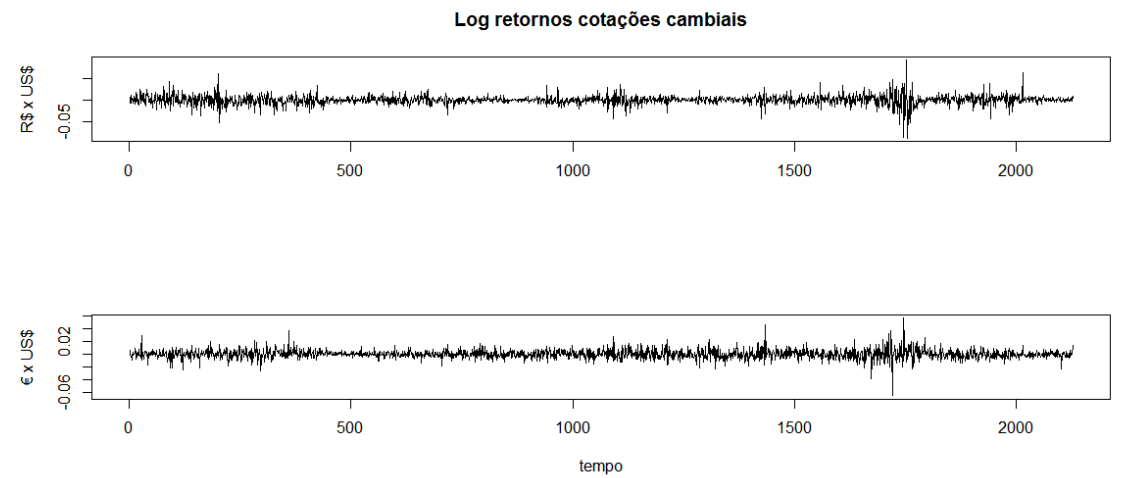

Figura 5.5: Log retorno do Real e do Euro precificados em Dólares

Analisando a função de autocorrelação (acf) e a função de autocorrelação parcial (pacf) na Figura 5.6, notamos que há evidências de que o para cada uma das séries temporais de retornos é $\operatorname{GARCH}(1,1)$.
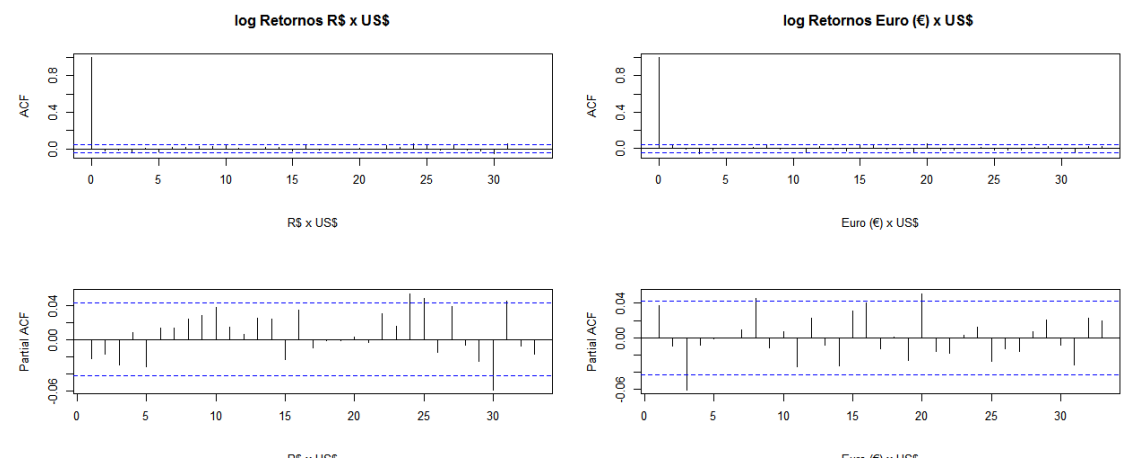

Figura 5.6: Acf e pacf dos log retornos do Real e do Euro

No entanto, quando tomamos o quadrado dos retornos e calculamos a acf e a pacf na 
Figura 5.7, notamos que a volatilidade não é constante. É possível notar que os quadrados estão correlacionados como esperado de retornos financeiros.
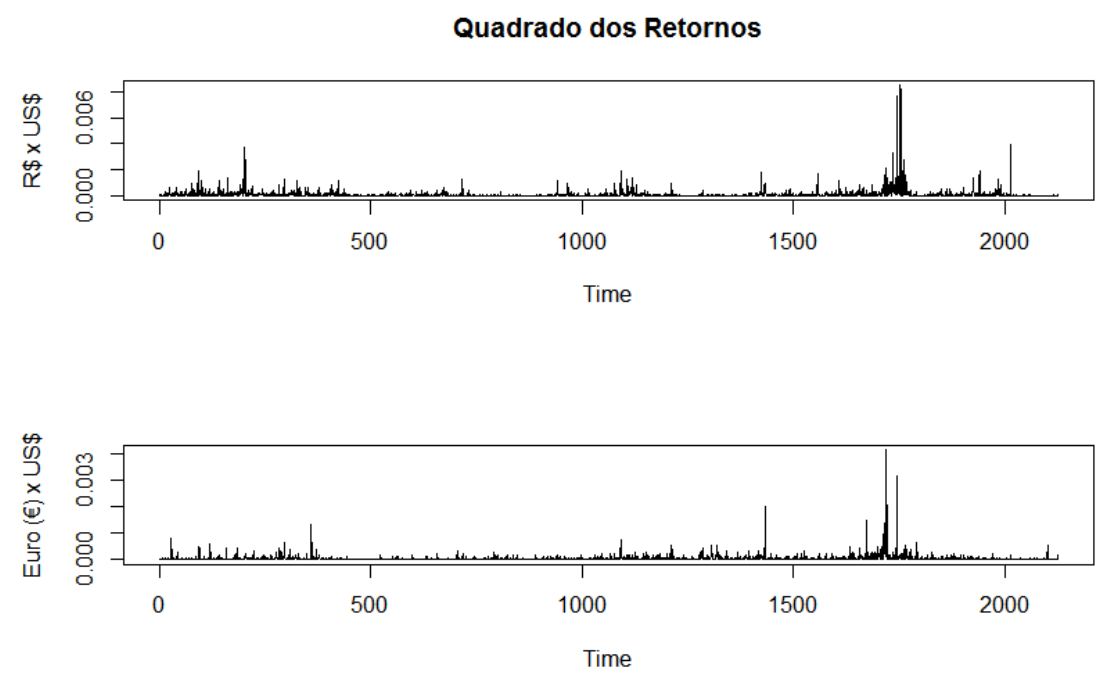

Figura 5.7: Quadrado do log retorno
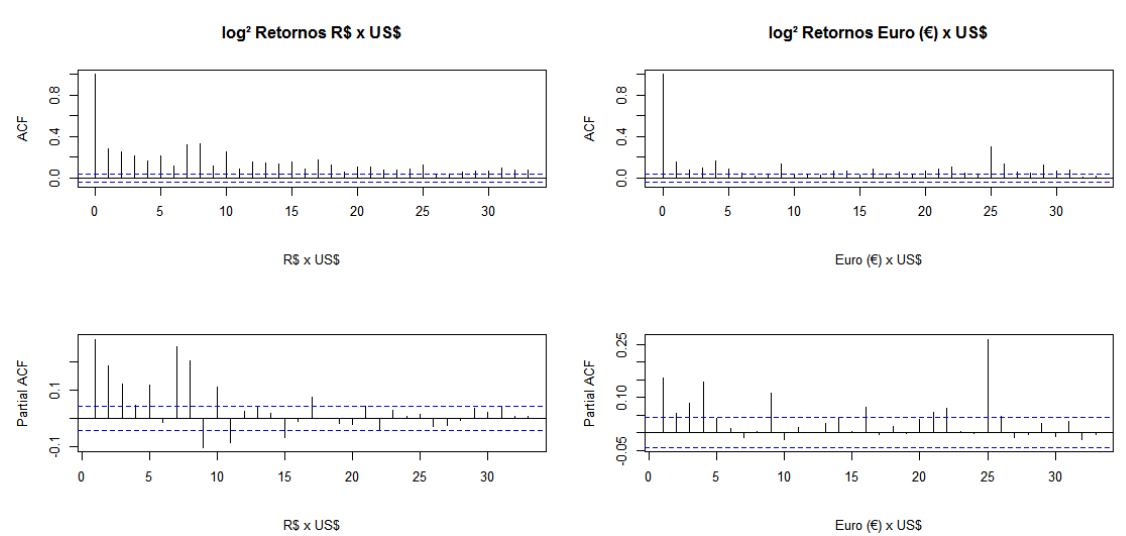

Figura 5.8: Acf e pacf dos quadrados dos log retornos

Neste contexto, usamos o modelo $\operatorname{GARCH}(1,1)$ para os retornos e então estimamos a dependência entre as duas séries temporais. Mais precisamente, 
a) Série da cotação do Real em Dólar

$$
\begin{aligned}
X_{t} & =1,19 \times 10^{-4}+\epsilon_{t} \\
\epsilon_{t} & =\sigma_{t} Z_{t}, Z_{t} \sim N(0,1) \\
\sigma_{t}^{2} & =7,65 \times 10^{-7}+0,11 \epsilon_{t-1}^{2}+0,892 \sigma_{t-1}^{2} .
\end{aligned}
$$

ou seja, $X_{t} \mid \mathcal{F}_{t-1} \sim N\left(1,19 \times 10^{-4} ; \sigma_{t}\right)$.

b) Série da cotação do Euro em Dólar

$$
\begin{aligned}
X_{t} & =1,57 \times 10^{-5}+\epsilon_{t} \\
\epsilon_{t} & =\sigma_{t} Z_{t}, Z_{t} \sim N(0,1) \\
\sigma_{t}^{2} & =2,53 \times 10^{-7}+0,04 \epsilon_{t-1}^{2}+0,953 \sigma_{t-1}^{2} .
\end{aligned}
$$

ou seja, $X_{t} \mid \mathcal{F}_{t-1} \sim N\left(1,57 \times 10^{-5} ; \sigma_{t}\right)$.

Agora vamos realizar uma aplicação da metodologia proposta nos resíduos das séries ajustadas do modelo GARCH(1,1). Ajustamos as cópulas Normal e Clayton, utilizando $J=5$ para Haar e $n=32$ para a ordem do Polinômio de Taylor. Comparamos a qualidade de ajuste pelo critério do menor AIC e BIC. A Tabela 5.9 mostra o AIC e BIC dos modelos ajustados. Podemos notar que a abordagem que melhor se ajusta aos dados é a abordagem de cópula Clayton variante no tempo, empregando a aproximação polinomial de Taylor.

Tabela 5.9: AIC e BIC para o Ajuste das Cópulas

\begin{tabular}{llrr}
\hline Copula & Abordagem & AIC & BIC \\
\hline normal & constante & $-31,6914$ & $-26,0668$ \\
& Haar & $-67,2988$ & 112,6889 \\
& polinomial & 10,8856 & 196,4980 \\
\hline \hline Clayton & constante & 2,4260 & 8,05066 \\
& Haar & $-7089,2837$ & $-6909,2958$ \\
& polinomial & $\mathbf{- 7 9 4 7 , 1 2 0 5}$ & $\mathbf{- 7 7 6 1 , 5 0 8 1}$ \\
\hline
\end{tabular}


As estimativas para $\theta_{t}$ e $\tau_{t}$ estão apresentadas na Figura 5.9 e 5.10 respectivamente. Note que a medida de dependência Tau de Kendall é não negativa no período analisado, indicando que quando o retorno do Euro aumenta (ou diminui) o retorno do Real tende a aumentar (diminuir). Além disso, podemos notar que durante a maior parte do período analisado o Tau de Kendall esteve abaixo de 0,35, o que significa uma fraca associação entre o retorno do Euro e do Real, e no final do período houve um aumento acentuado da associação entre os dois retornos.

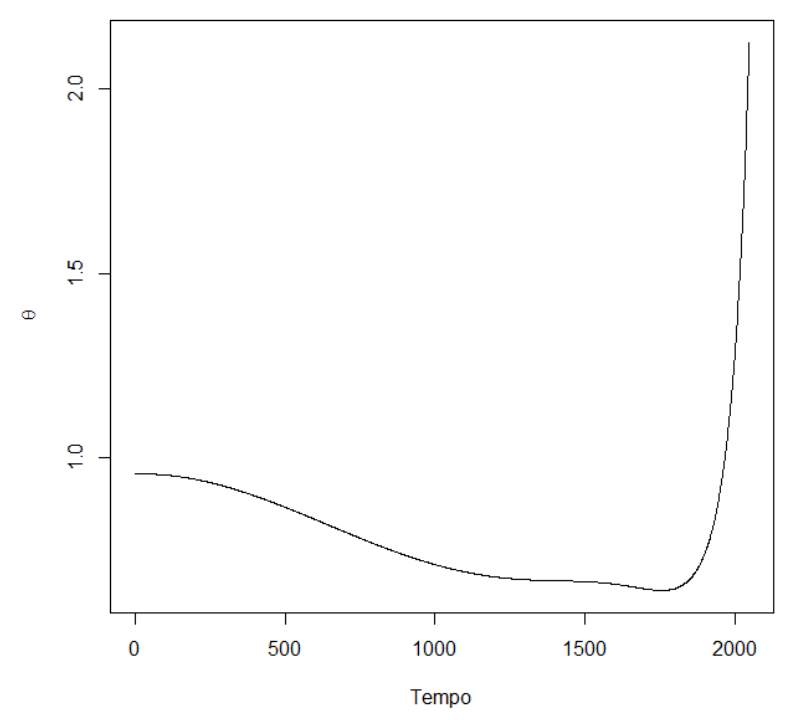

Figura 5.9: $\hat{\theta}_{t}$ para a cópula Clayton

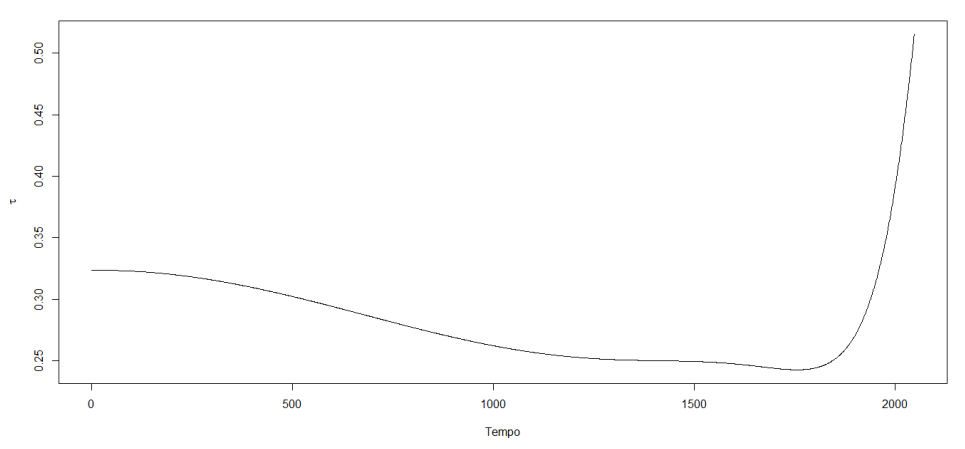

Figura 5.10: $\hat{\tau}$ de Kendall cópula Clayton 


\section{Capítulo 6}

\section{Conclusão}

Neste trabalho, propomos uma nova abordagem para cópula variante no tempo, adaptando a proposta de cópula condicional de Abegaz et al. (2012). Dentre os métodos de estimação da abordagem variante no tempo o polinômio de Taylor apresentou o melhor resultado geral. A cópula Normal mostrou-se mais adequada para os dados simulados. No entanto, a cópula Clayton apresentou melhor ajuste para os dados aplicados.

Acreditamos que o fraco desempenho do método Haar na simulação se dá por conta de estarmos aproximando uma função suave. Na aplicação, notamos que houve uma melhora em seu desempenho e, portanto, faz-se necessário investigar seu desempenho em outras situações, dentro da abordagem variante no tempo.

\section{Estudos futuros}

Como tópicos para estudos futuros, sugerimos:

- A investigação de outros modelos de cópulas presentes na literatura como em Nelsen (2013) e Denuit et al. (2006).

- O estudo das propriedades assintóticas do estimador de máxima verossimilhança no contexto clássico. 
- A utilização da abordagem clássica para a estimação dos coeficientes das bases de aproximação de funções.

- O uso de outras bases de funções na estimação de $\theta_{t}$ tais como Ondaletas de Daubechies, Splines, Fourier e outros.

- Estudos de simulação considerando funções $h(t)$ com domínios $[-1,1]$, como por exemplo: $\operatorname{sen}(t) e \cos (t)$. 


\section{Apêndice A}

\section{Código R}

Neste apêndice, disponibilizamos todo o código fonte em R produzido neste trabalho tornando possível a reprodutibilidade. Organizamos este texto em duas seções: Caso Clayton e Caso Normal. Cada Seção tem quatro subseções: parâmetro constante, Haar (base ondaletas Haar), Taylor (base de polinômios) e Kernel.

\section{A.1 Caso Clayton}

\section{A.1.1 Constante}

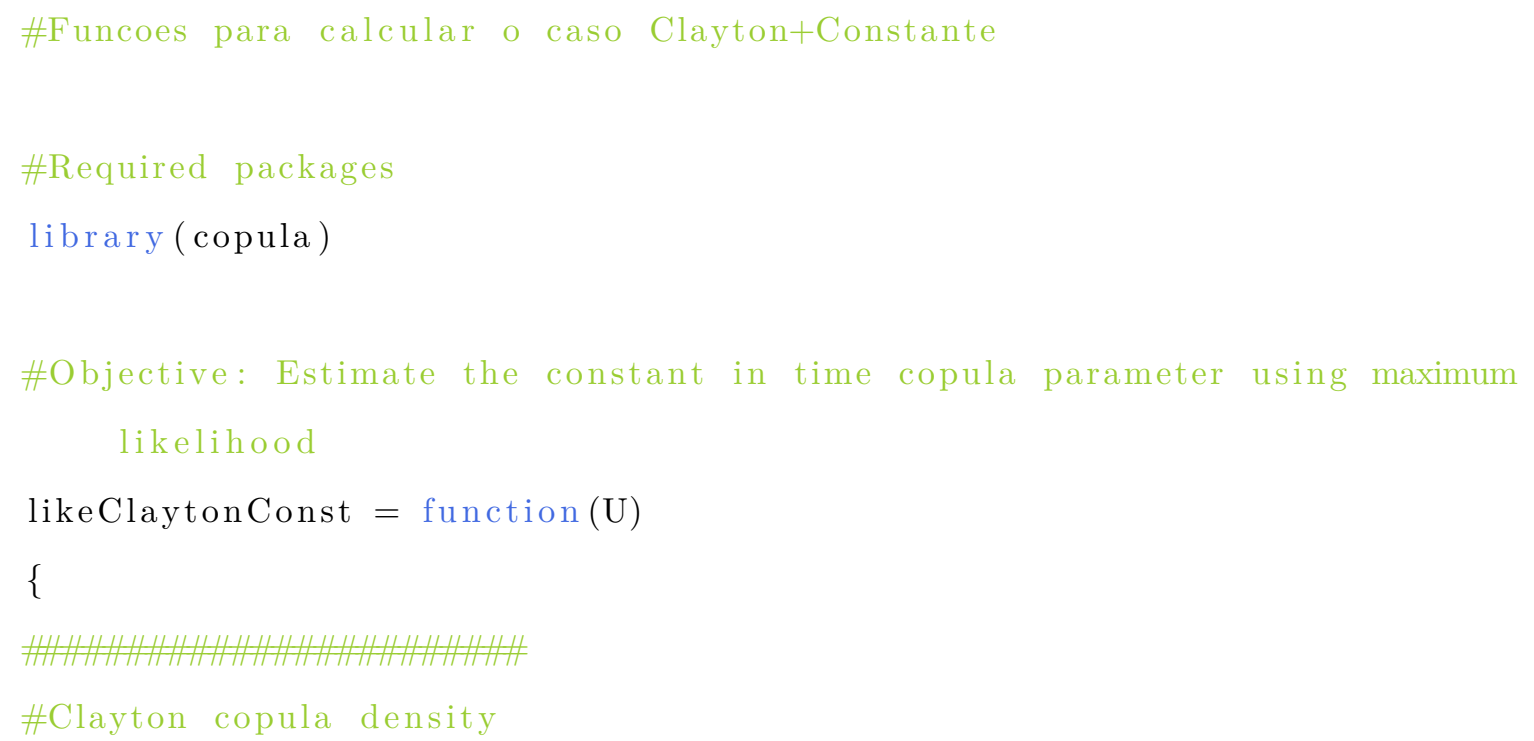




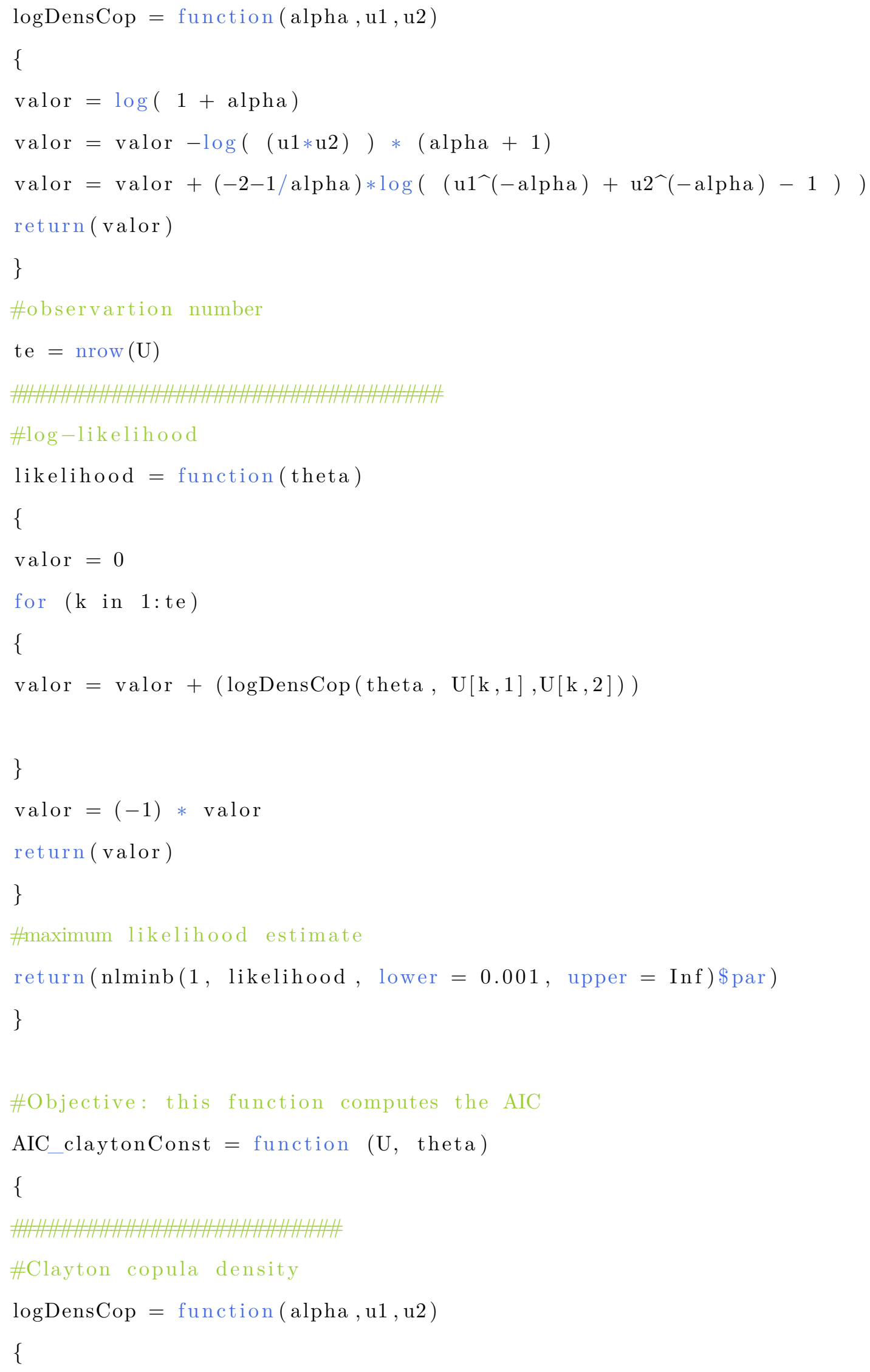




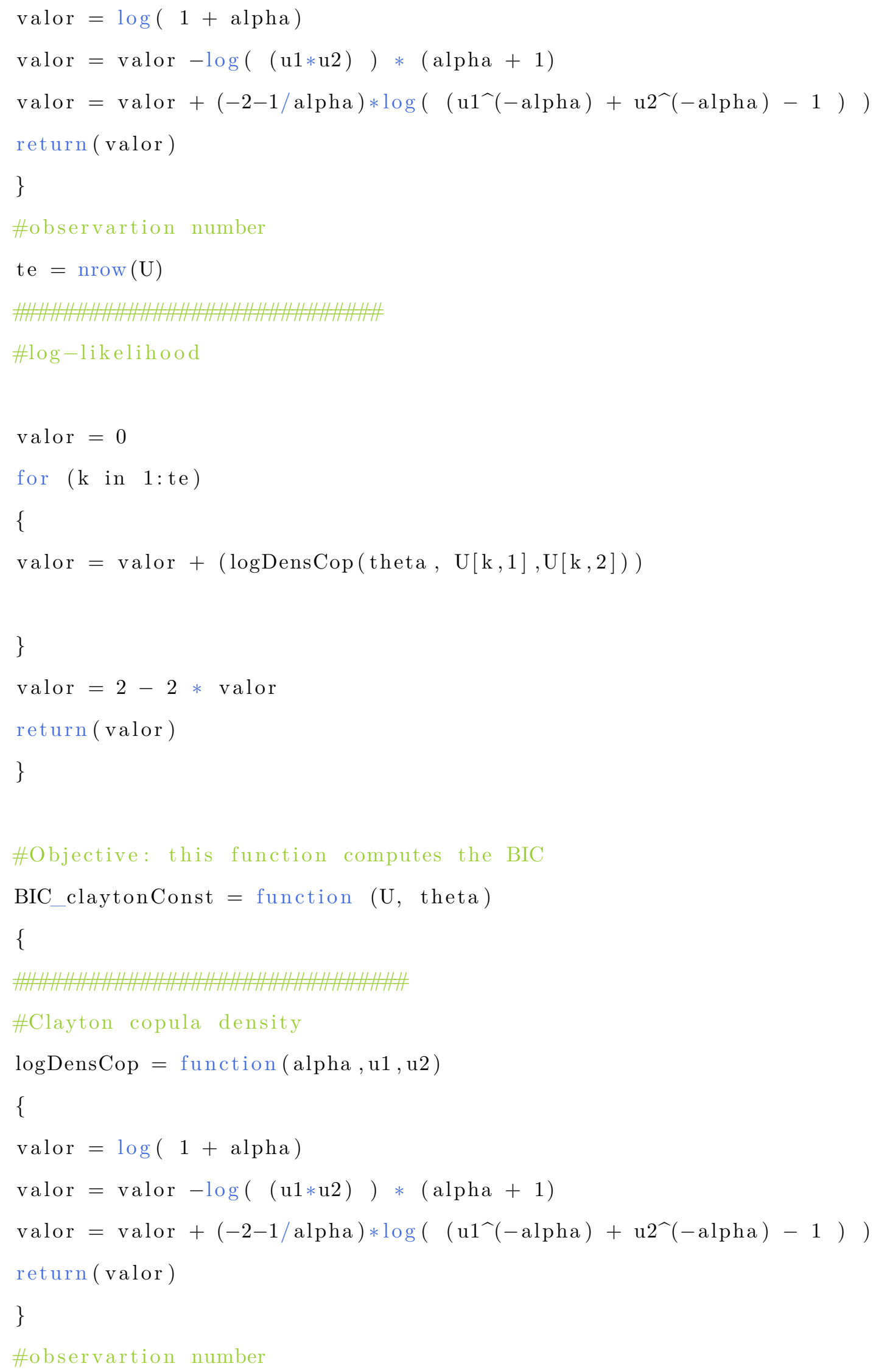


$\mathrm{te}=\operatorname{nrow}(\mathrm{U})$

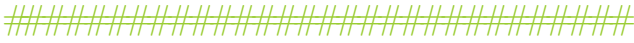

\#log-likelihood

valor $=0$

for $(\mathrm{k}$ in $1: \mathrm{te})$

\{

valor $=$ valor $+(\log \operatorname{Dens} \operatorname{Cop}($ theta $, \mathrm{U}[\mathrm{k}, 1], \mathrm{U}[\mathrm{k}, 2]))$

\}

valor $=\log ($ te $)-2 *$ valor

return (valor)

\}

\section{A.1.2 Haar}

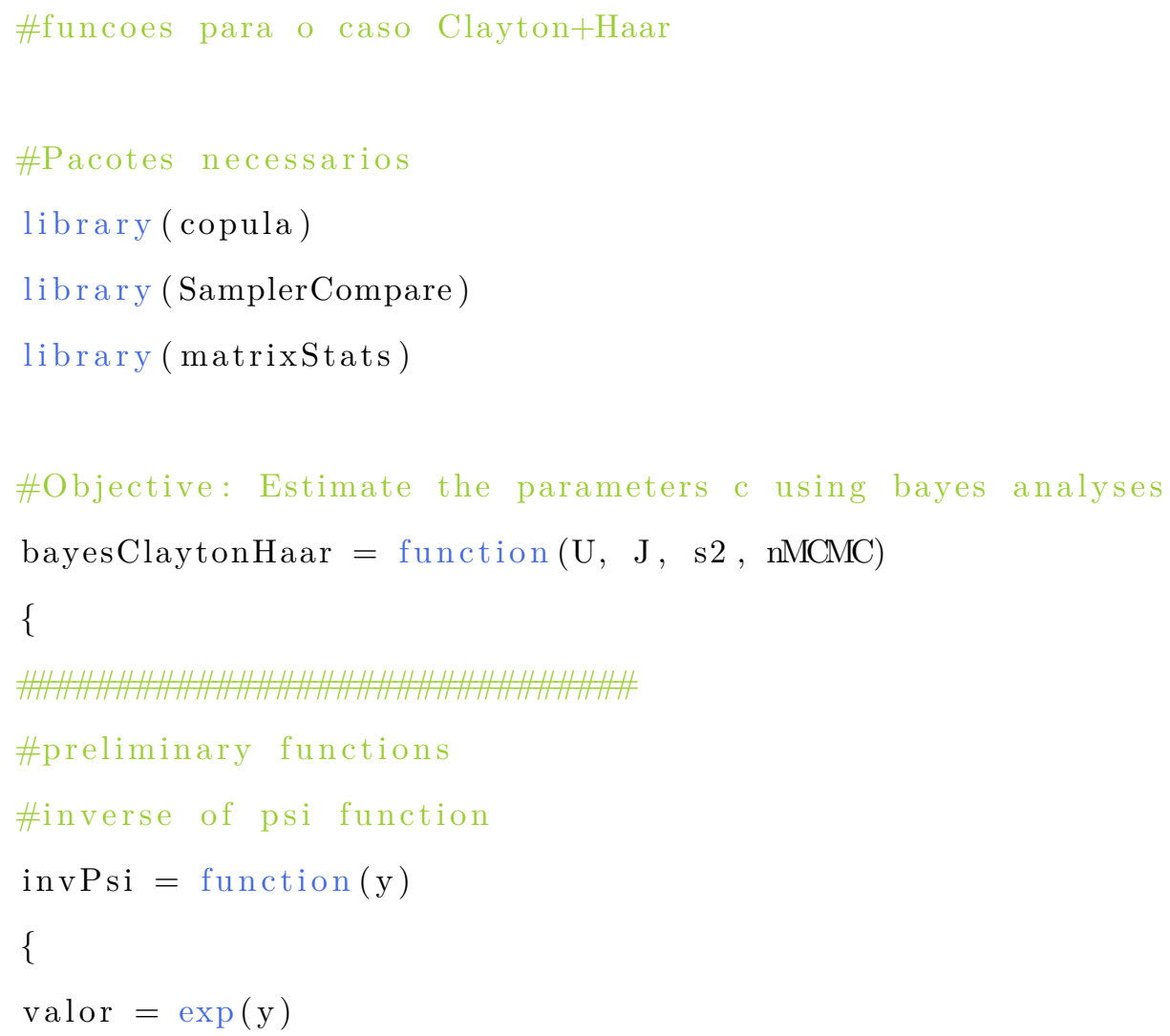




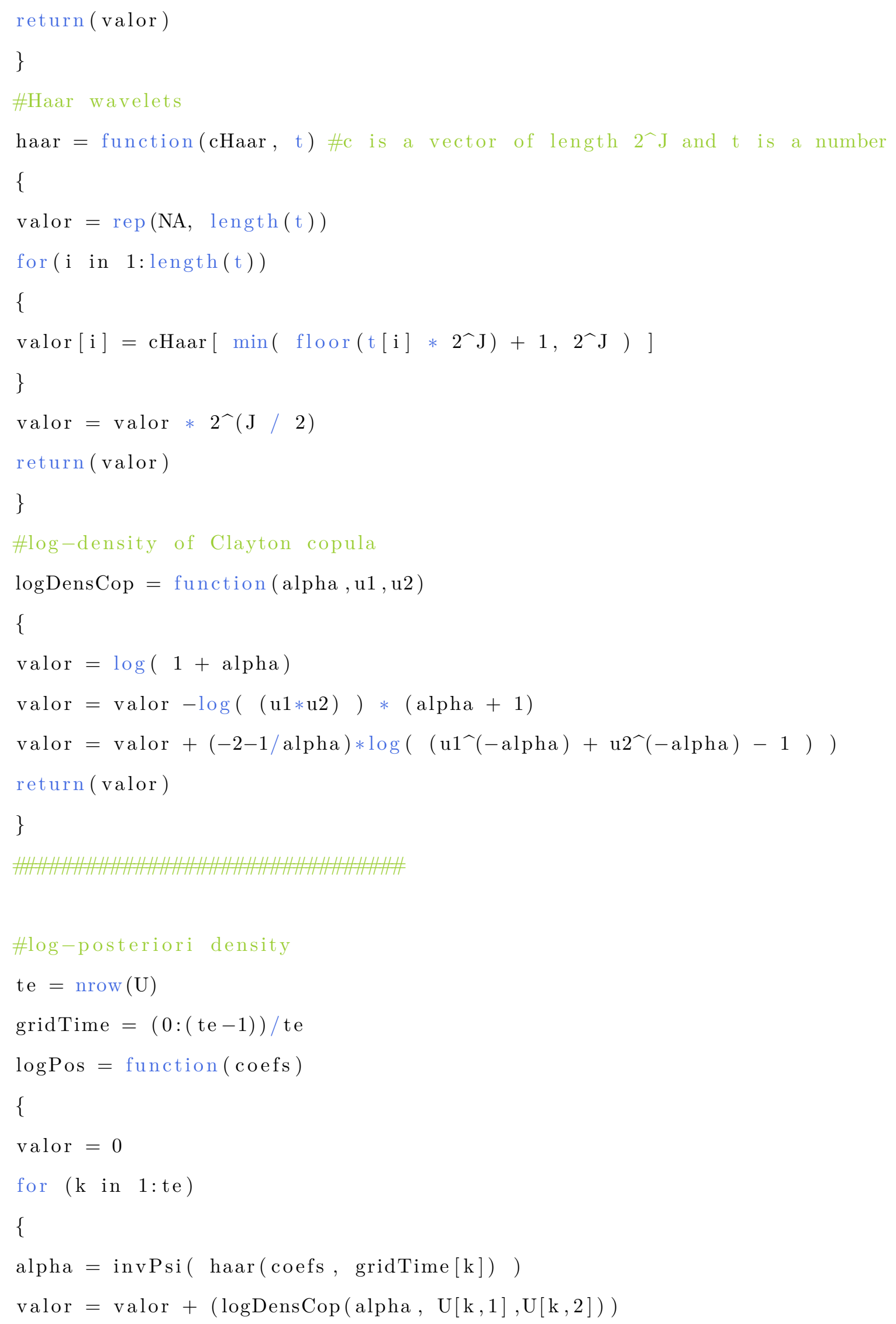




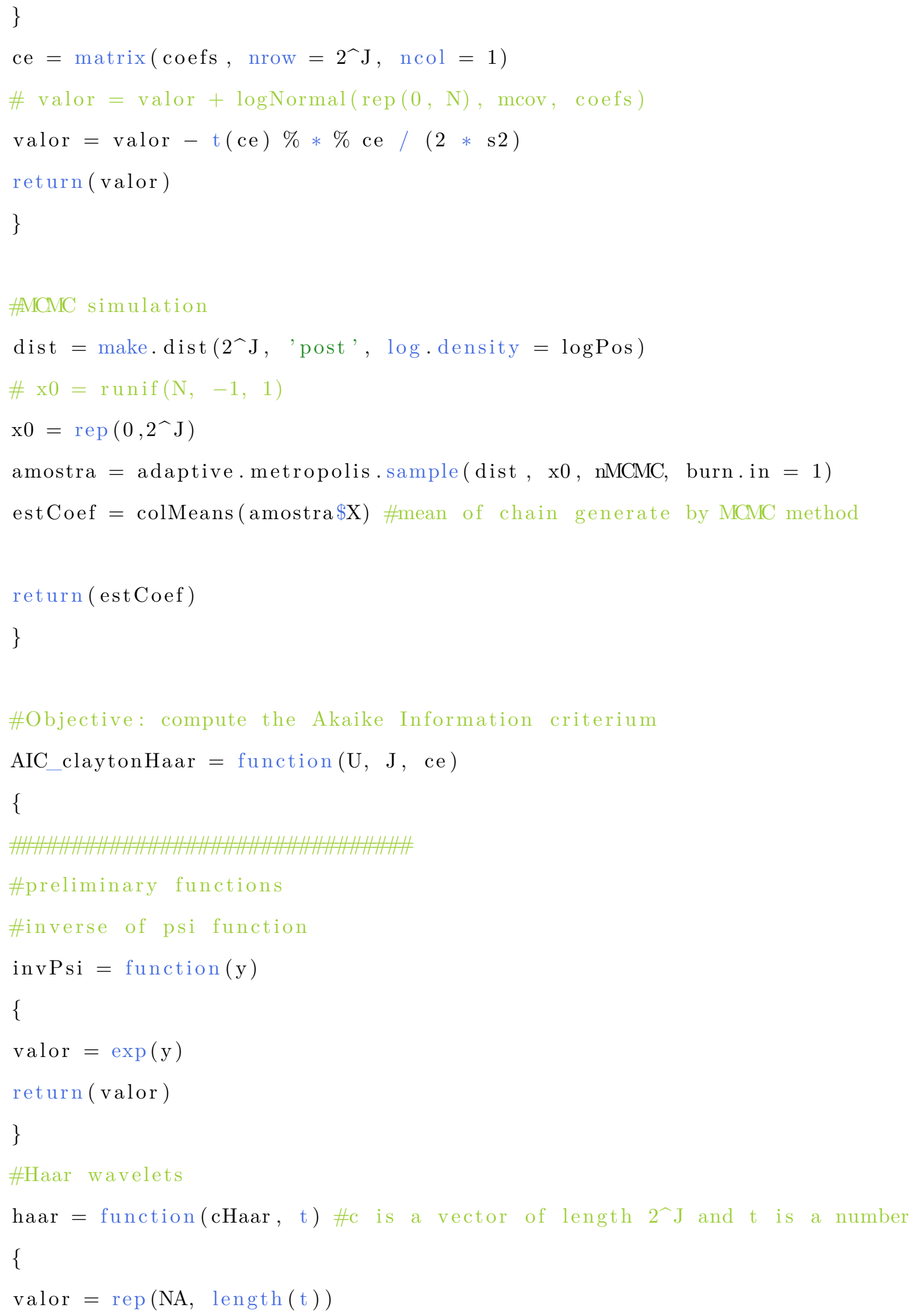




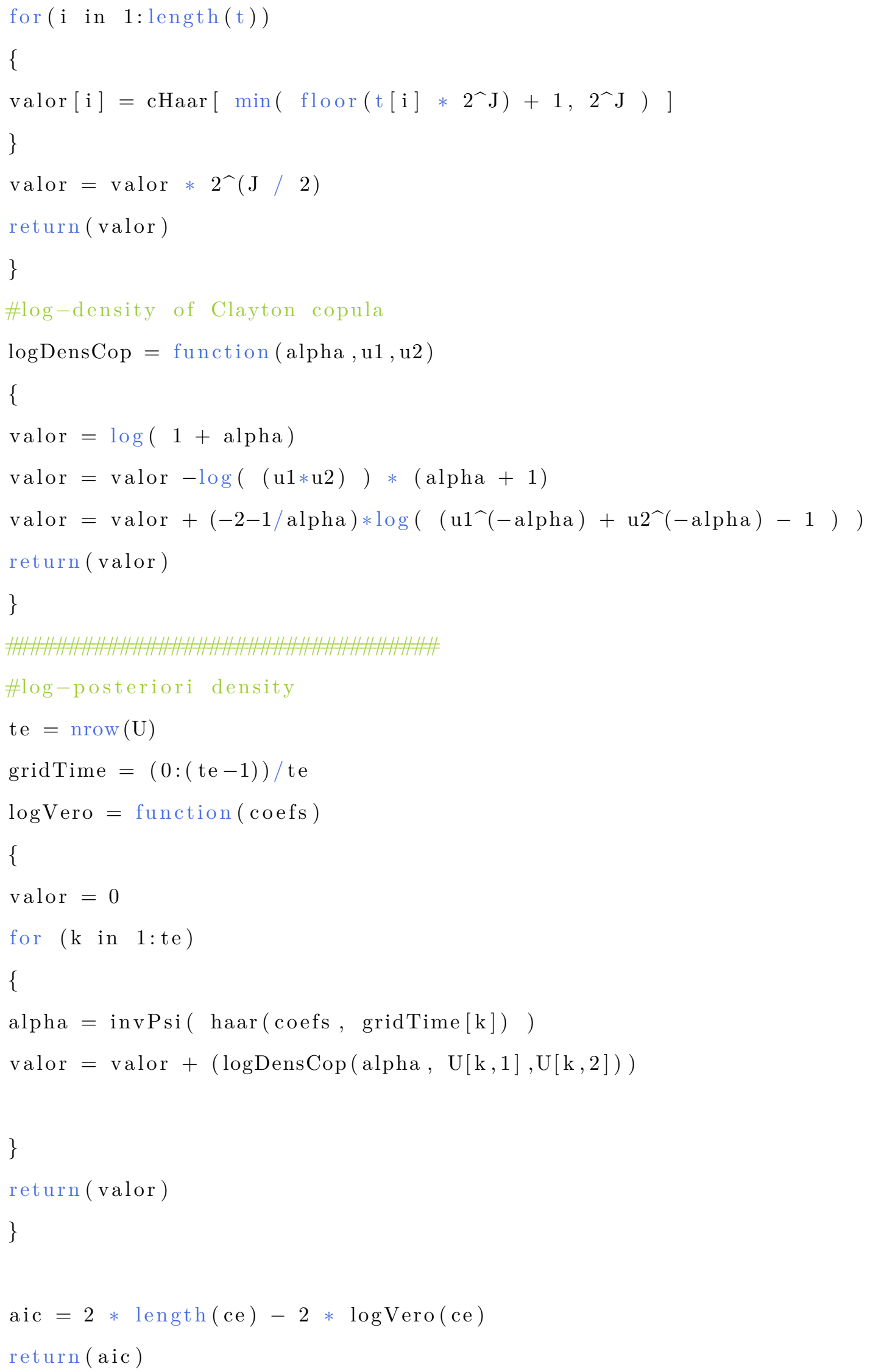


\}

\#Objective: computes the Bayesian Information Criterium

BIC_claytonHaar = function $(\mathrm{U}, \mathrm{J}$, ce $)$

\{

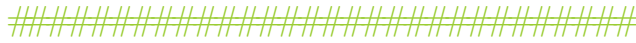

\#preliminary functions

\#inverse of psi function

invPsi $=$ function $(y)$

\{

valor $=\exp (y)$

return(valor)

\}

\#Haar wavelets

haar = function(cHaar, $\mathrm{t}) \# \mathrm{c}$ is a vector of length $2^{\wedge} \mathrm{J}$ and $\mathrm{t}$ is a number \{

valor $=\operatorname{rep}(\mathrm{NA}$, length $(\mathrm{t}))$

for ( $\mathrm{i}$ in $1: \operatorname{length}(\mathrm{t})$ )

\{

valor $[\mathrm{i}]=\operatorname{cHaar}\left[\min \left(\operatorname{floor}\left(\mathrm{t}[\mathrm{i}] * 2^{\wedge} \mathrm{J}\right)+1,2^{\wedge} \mathrm{J}\right)\right]$

\}

valor $=$ valor $* 2$ ^ $(\mathrm{J} / 2)$

return(valor)

\}

\#log-density of Clayton copula

$\log$ DensCop $=$ function $($ alpha $, \mathrm{u} 1, \mathrm{u} 2)$

\{

valor $=\log (1+$ alpha $)$

valor $=$ valor $-\log ((\mathrm{u} 1 * \mathrm{u} 2)) *(\operatorname{alpha}+1)$

valor $=$ valor $+(-2-1 /$ alpha $) * \log \left(\left(u 1^{\wedge}(-\right.\right.$ alpha $)+u 2^{\wedge}(-$ alpha $\left.\left.)-1\right)\right)$

return(valor)

\}

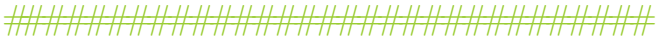




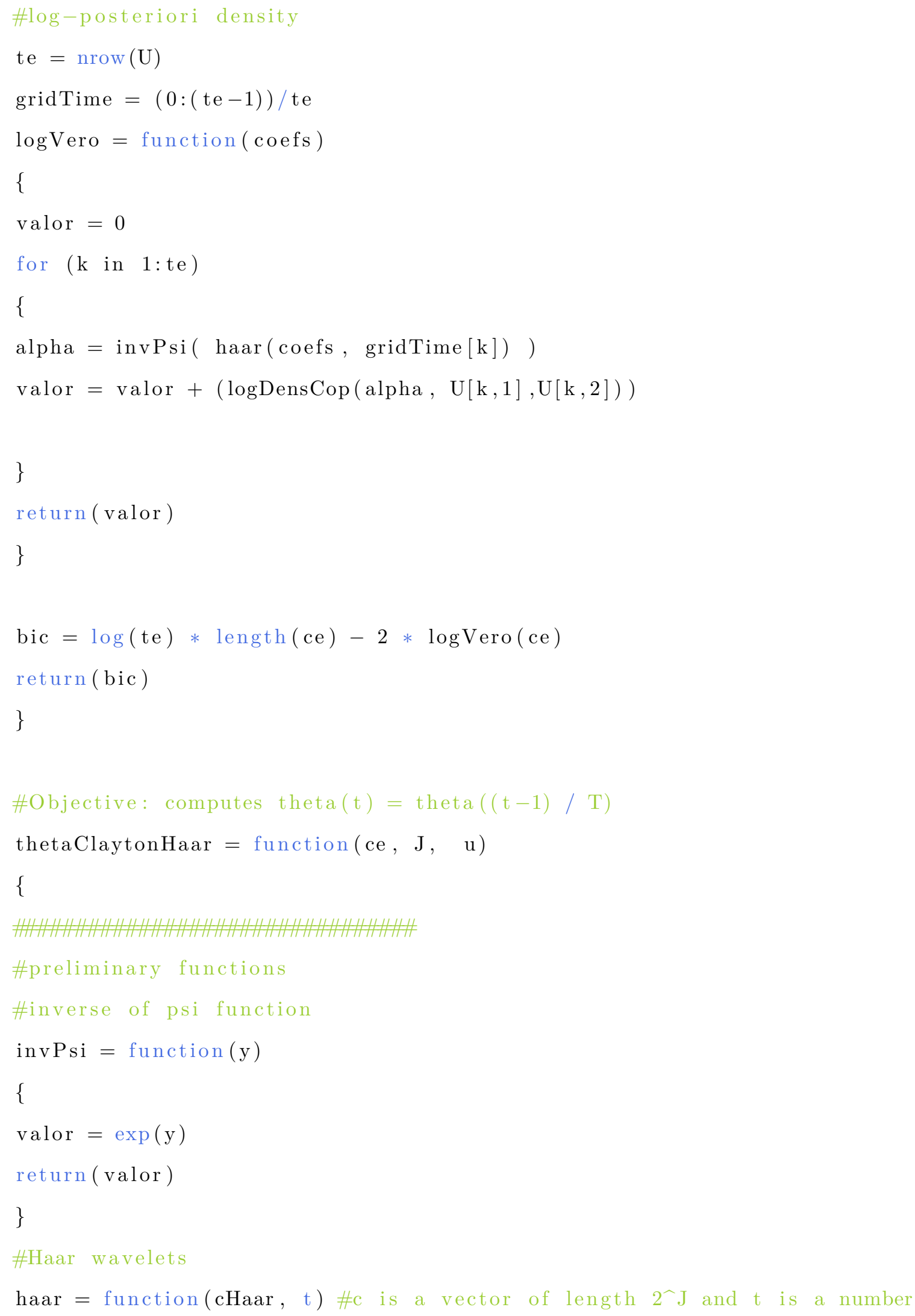


\{

valor $=\operatorname{rep}(\mathrm{NA}$, length $(\mathrm{t}))$

for (i in 1 :length $(t)$ )

\{

valor $[\mathrm{i}]=\operatorname{cHaar}\left[\min \left(\operatorname{floor}\left(\mathrm{t}[\mathrm{i}] * 2^{\wedge} \mathrm{J}\right)+1,2^{\curlywedge} \mathrm{J}\right)\right]$

\}

valor $=$ valor $* 2$ ^ $(\mathrm{J} / 2)$

return(valor)

\}

\#log-density of Clayton copula

$\log$ DensCop $=$ function (alpha, $\mathrm{u} 1, \mathrm{u} 2)$

\{

valor $=\log (1+$ alpha $)$

valor $=$ valor $-\log ((\mathrm{u} 1 * \mathrm{u} 2)) *(\operatorname{alpha}+1)$

valor $=$ valor $+(-2-1 /$ alpha $) * \log \left(\left(u 1 \uparrow(-\right.\right.$ alpha $)+u 2^{\wedge}(-$ alpha $\left.\left.)-1\right)\right)$

return(valor)

\}

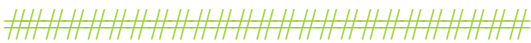

\#column vector with the theta values

output $=$ invPsi $($ haar $(c e, u))$

return (output)

\}

tauClaytonHaar $=$ function $(c e, \mathrm{~J}, \mathrm{u})$

\{

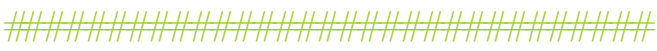

\#preliminary functions

\#inverse of psi function

invPsi $=$ function $(y)$

\{

valor $=\exp (\mathrm{y})$

return (valor) 


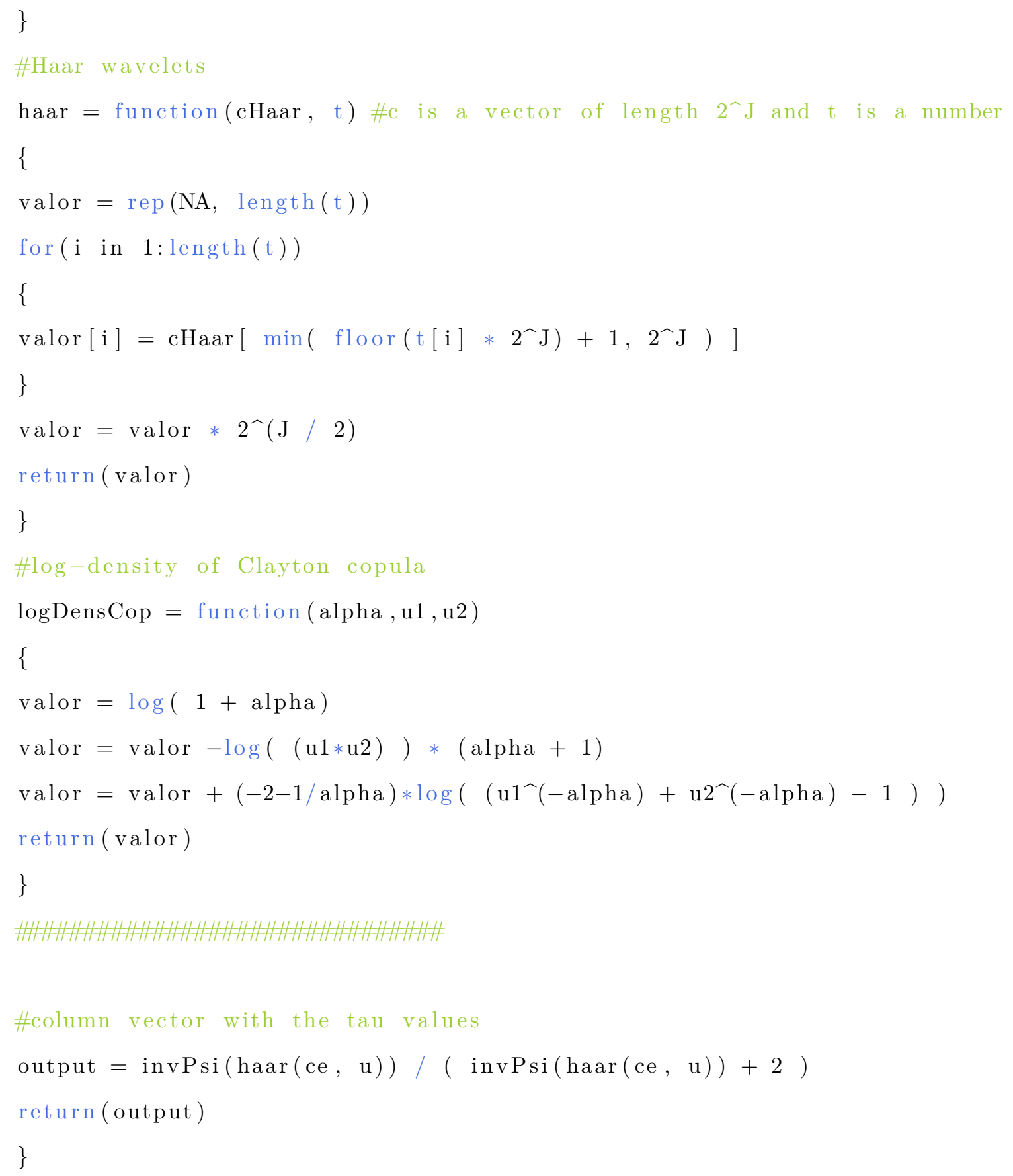

\section{A.1.3 Taylor}

\#Funcoes para o caso Clayton+Taylor 


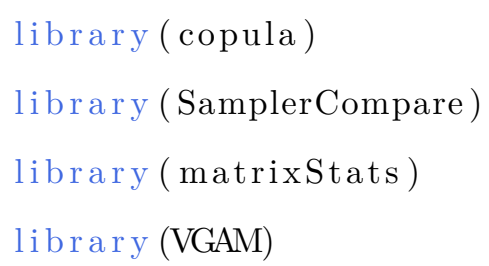




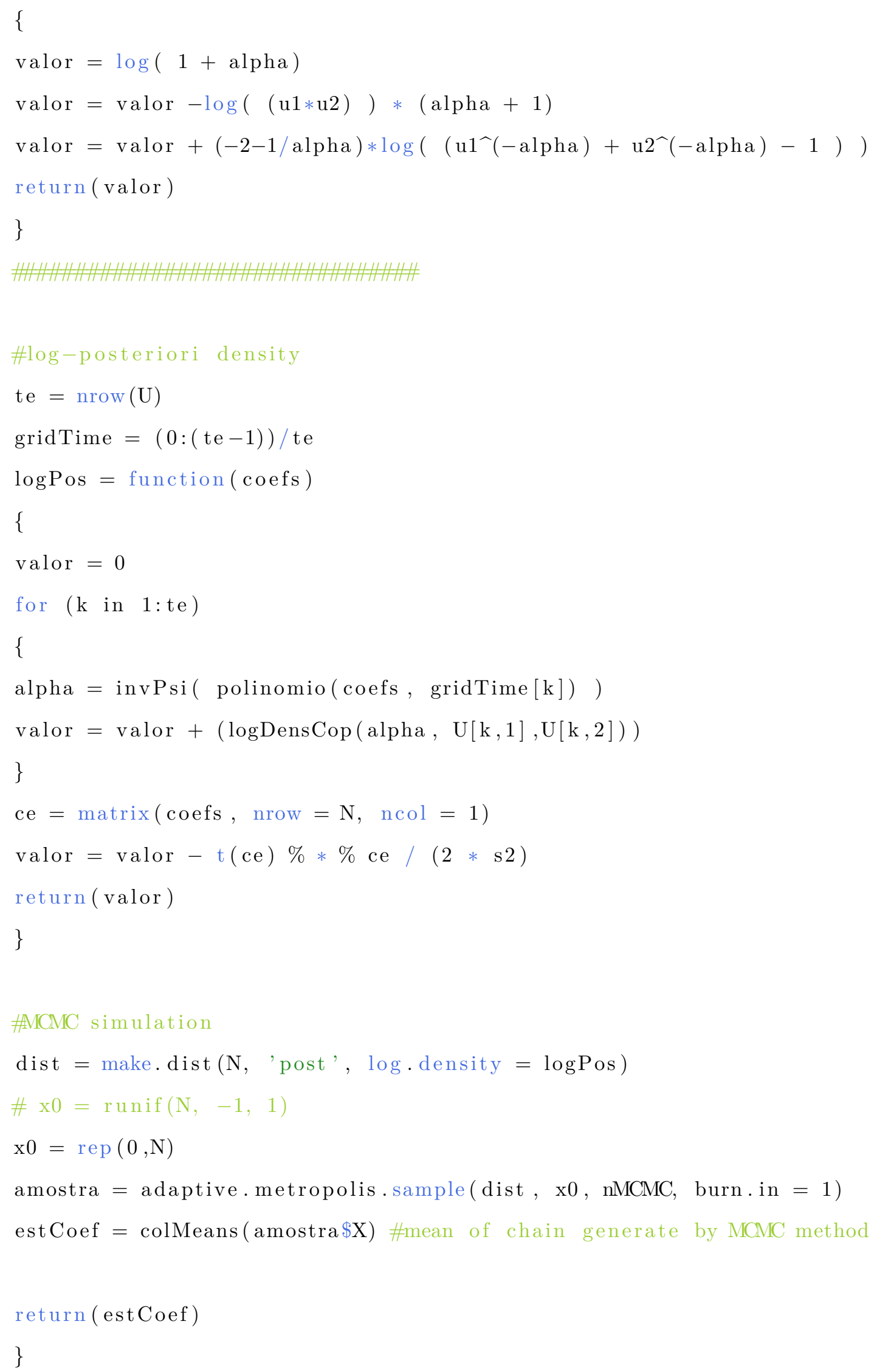




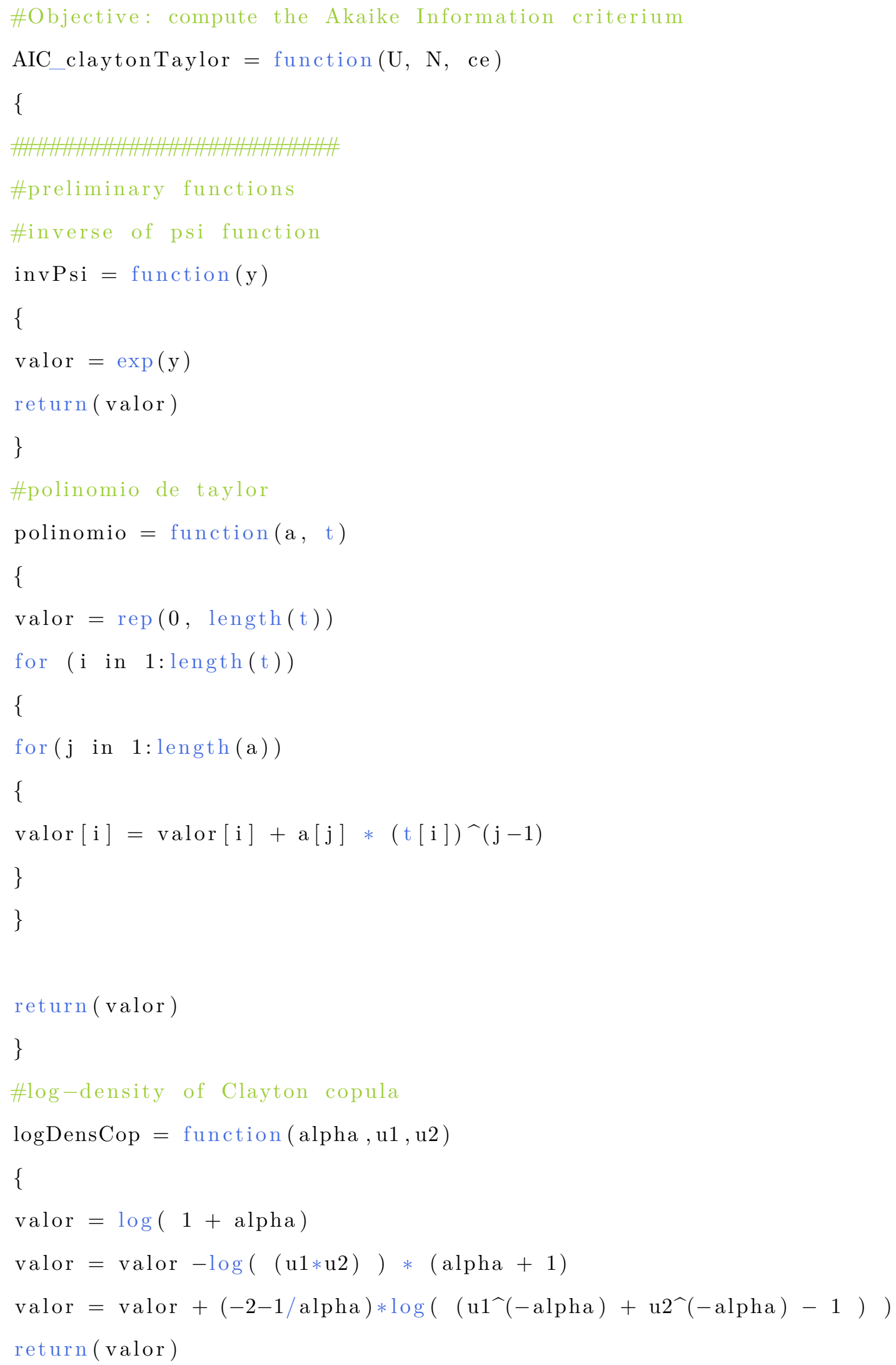


\}

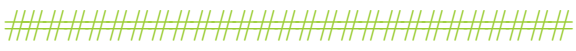

\#log-posteriori density

te $=\operatorname{nrow}(\mathrm{U})$

gridTime $=(0:($ te -1$)) /$ te

$\log$ Vero $=$ function $($ coefs $)$

\{

valor $=0$

for $(\mathrm{k}$ in $1:$ te $)$

\{

alpha $=$ invPsi $(\operatorname{polinomio}(\operatorname{coefs}, \operatorname{gridTime}[\mathrm{k}]))$

valor $=$ valor $+(\log \operatorname{DensCop}(\operatorname{alpha}, \mathrm{U}[\mathrm{k}, 1], \mathrm{U}[\mathrm{k}, 2]))$

\}

return (valor)

\}

aic $=2 *$ length $($ ce $)-2 * \log$ Vero $($ ce $)$

return (aic)

\}

\#Objective: computes the Bayesian Information Criterium

BIC_claytonTaylor $=$ function $(\mathrm{U}, \mathrm{N}$, ce $)$

\{

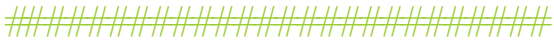

\#preliminary functions

\#inverse of psi function

invPsi $=$ function $(\mathrm{y})$

\{

valor $=\exp (\mathrm{y})$

return (valor)

\}

\#polinomio de taylor 


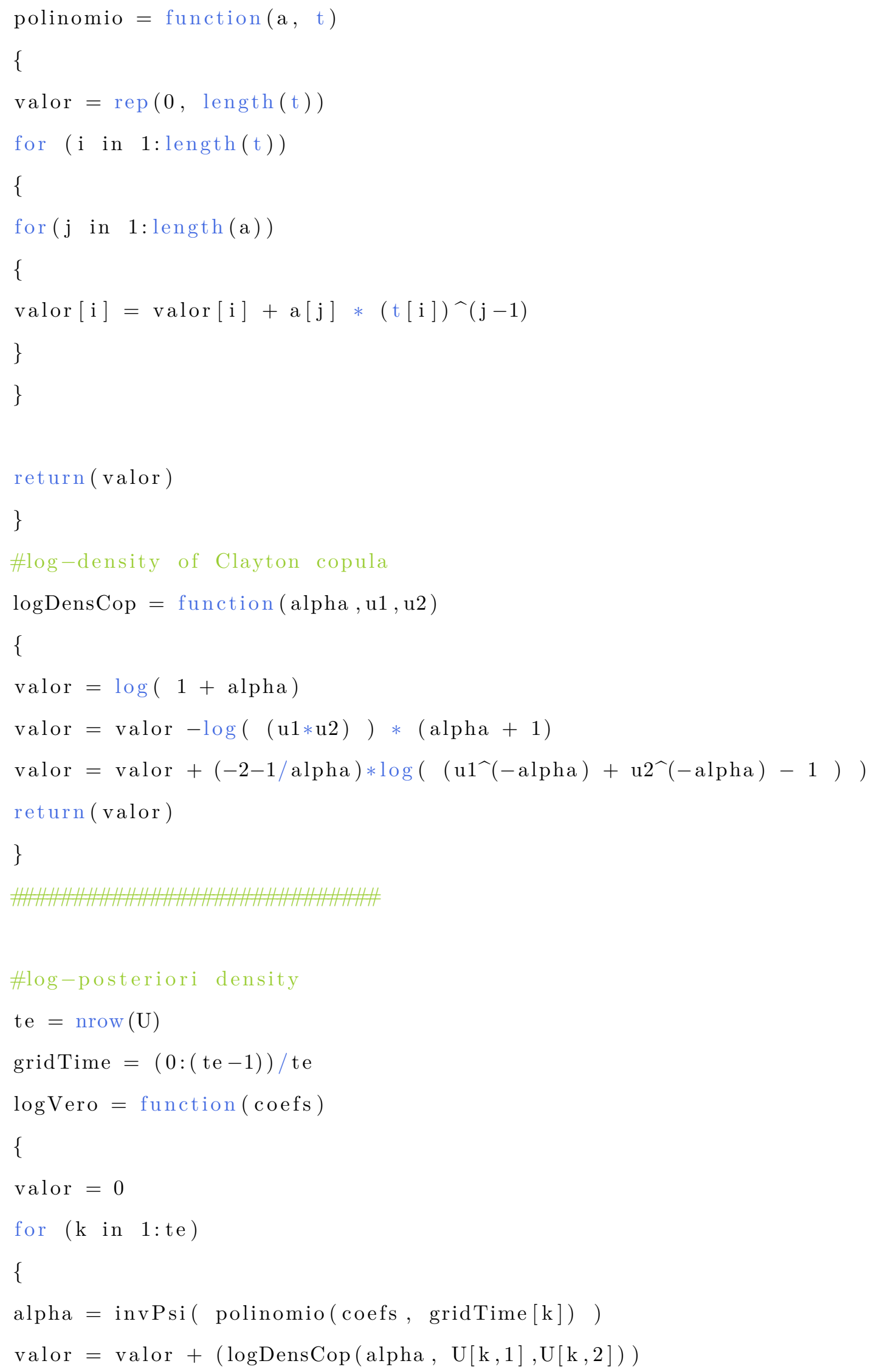


\}

return(valor)

\}

bic $=\log ($ te $) * \operatorname{length}($ ce $)-2 * \log$ Vero(ce)

return (bic)

\}

\#Objective: computes theta $(\mathrm{t})=$ theta $((\mathrm{t}-1) / \mathrm{T})$

thetaClaytonTaylor $=$ function $(\mathrm{ce}, \mathrm{N}, \mathrm{u})$

\{

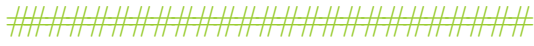

\#preliminary functions

\#inverse of psi function

invPsi $=$ function $(y)$

\{

valor $=\exp (\mathrm{y})$

return (valor)

\}

\#polinomio de taylor

polinomio $=$ function $(a, t)$

\{

valor $=\operatorname{rep}(0, \operatorname{length}(\mathrm{t}))$

for ( $\mathrm{i}$ in 1 : length $(\mathrm{t})$ )

\{

for $(j$ in $1: \operatorname{length}(\mathrm{a}))$

\{

valor $[\mathrm{i}]=\operatorname{valor}[\mathrm{i}]+\mathrm{a}[\mathrm{j}] *(\mathrm{t}[\mathrm{i}]) \wedge(\mathrm{j}-1)$

\}

\}

return (valor) 


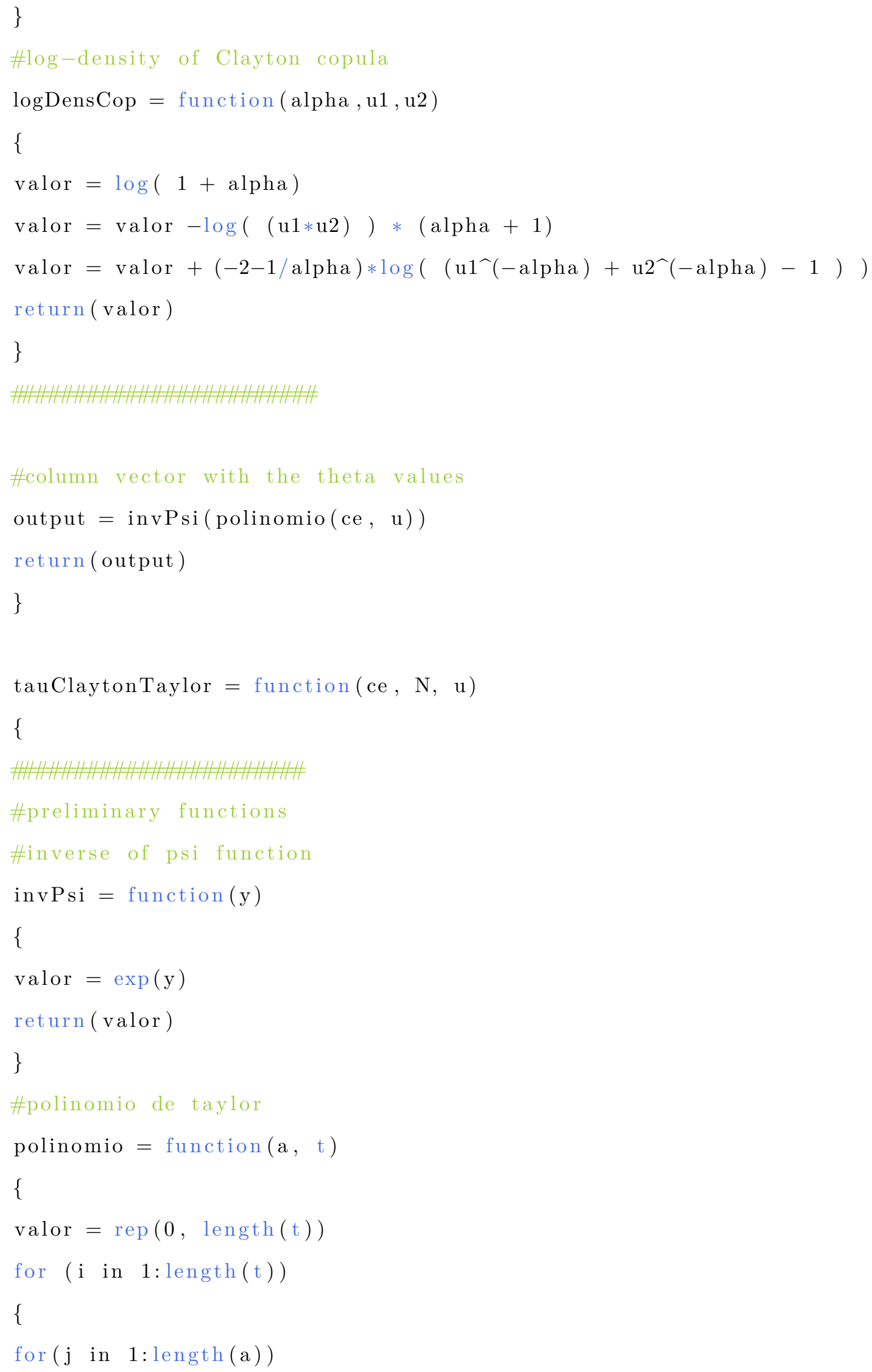




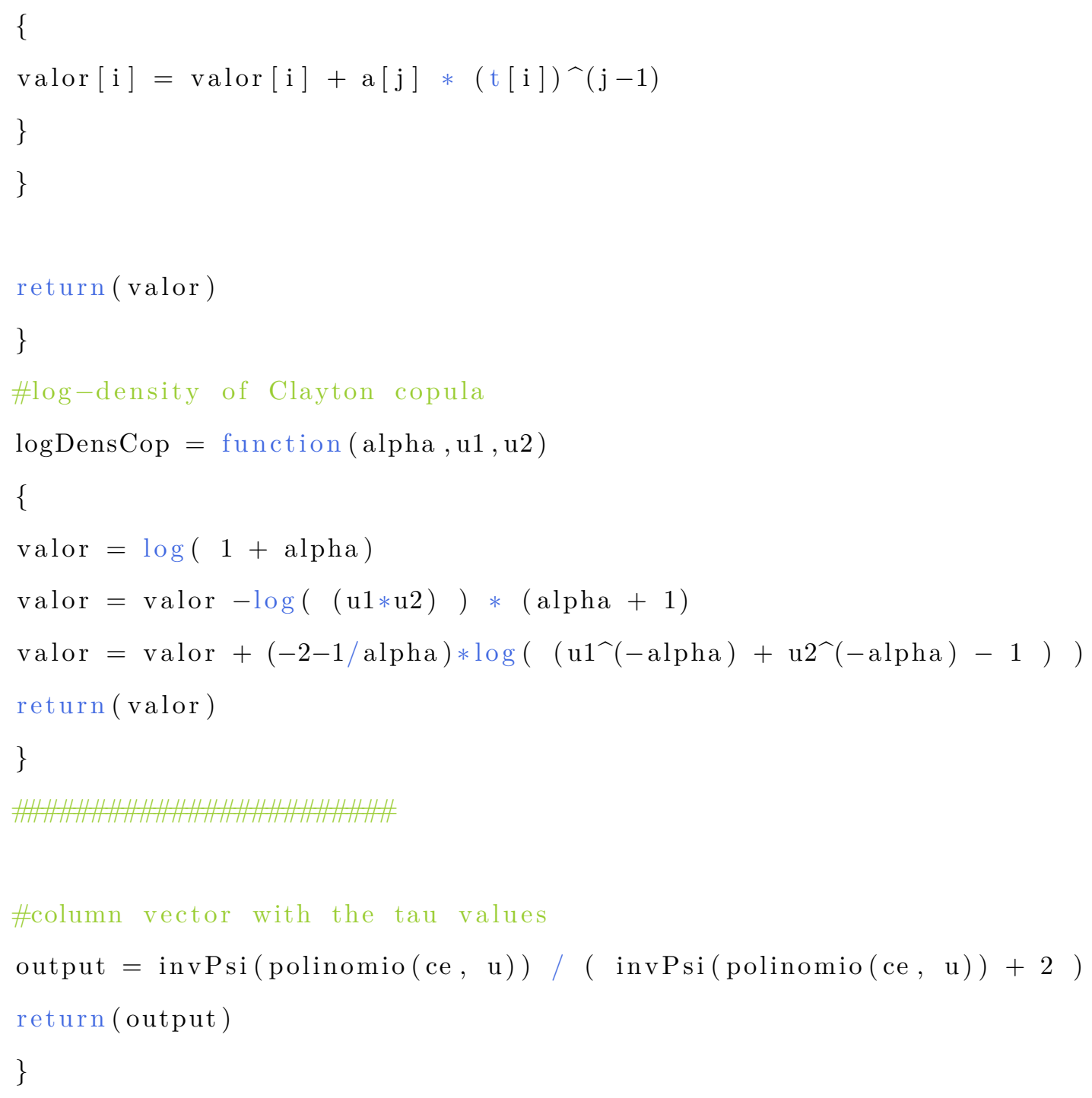

\section{A.2 Normal}

\section{A.2.1 Constante}

\#Funcoes para calcular o caso normal+Constante

\#Required packages

library (copula) 


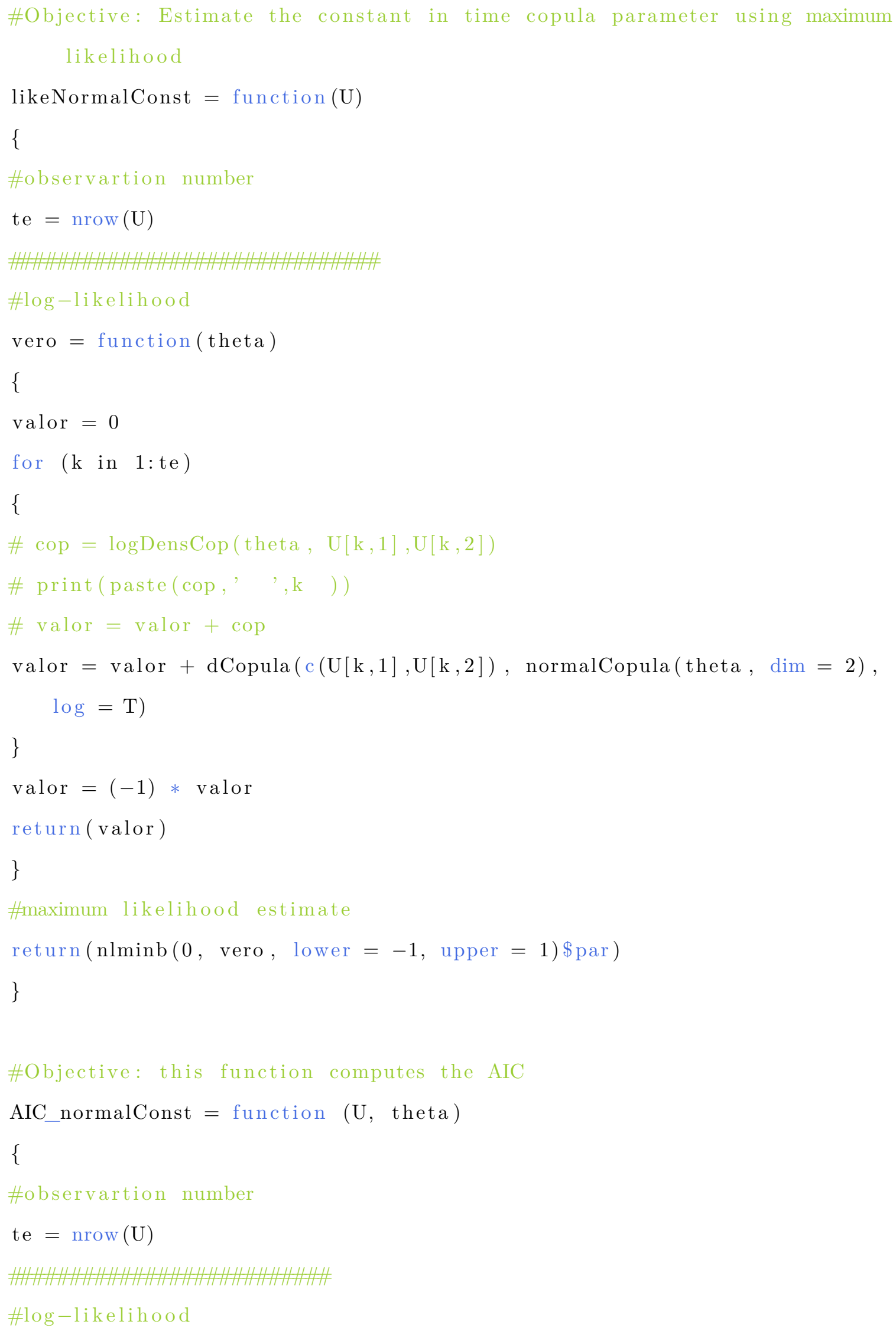




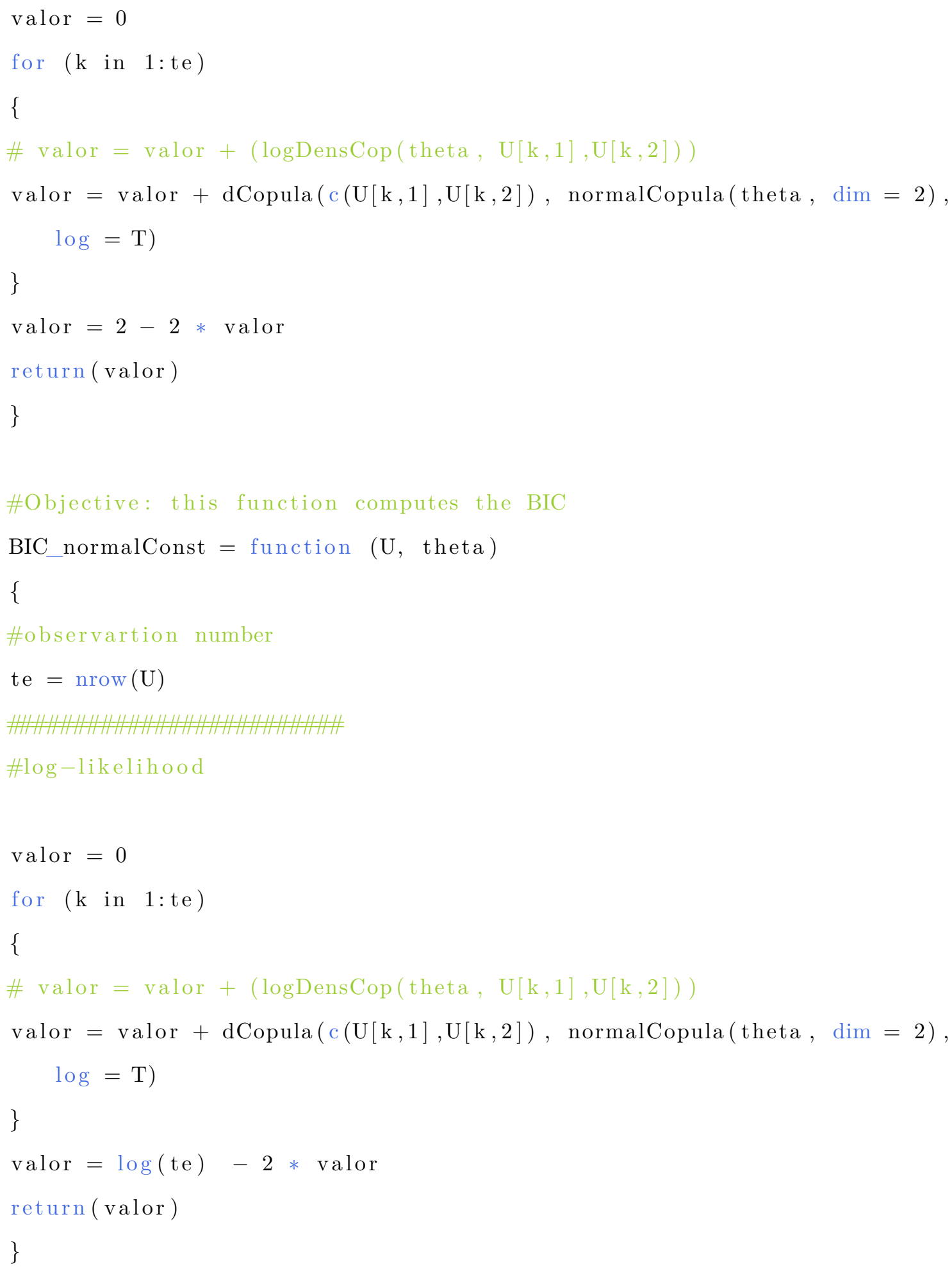

\section{A.2.2 Haar}




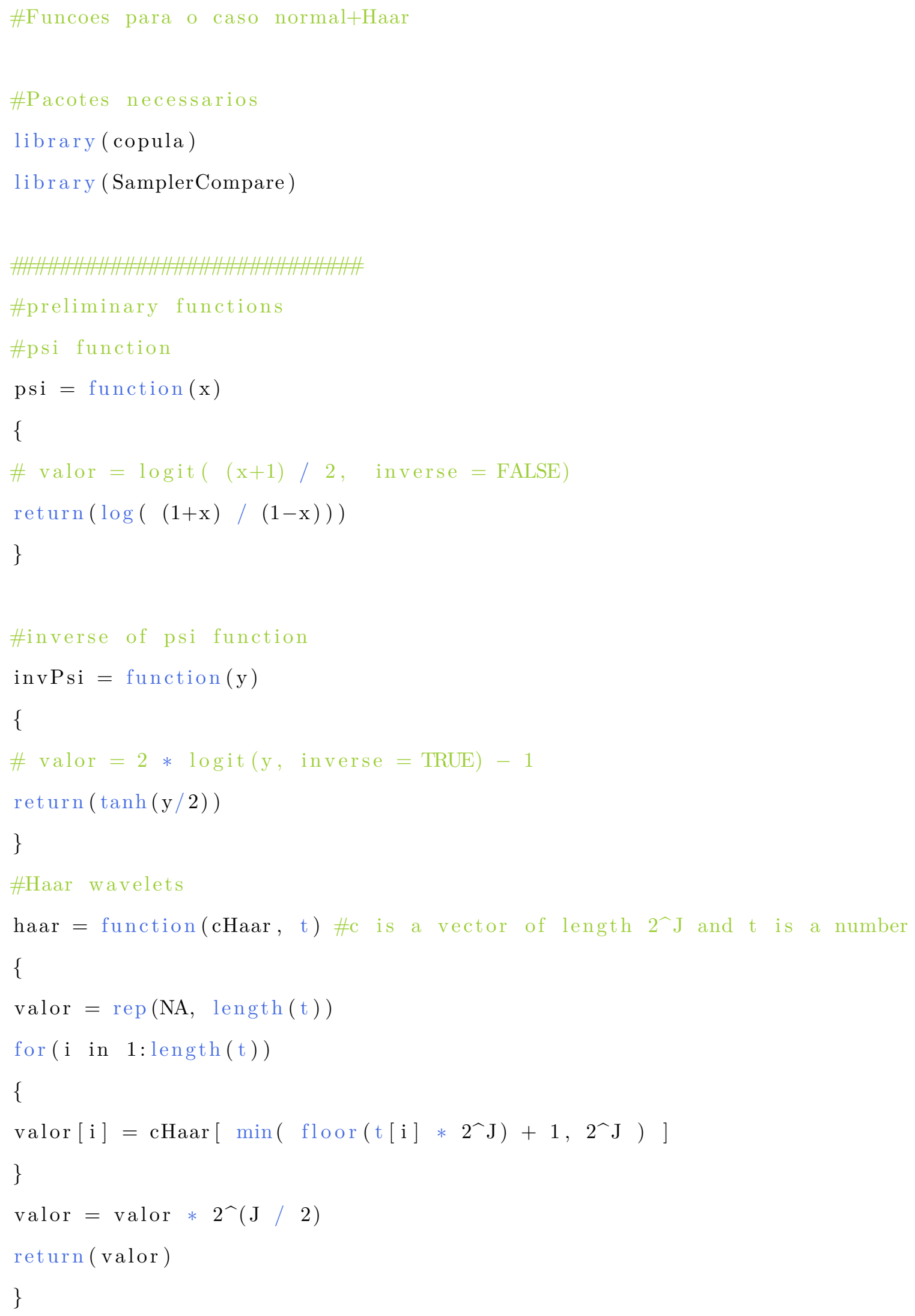


\#Objective: Estimate the parameters c using bayes analyses

bayesNormalHaar $=$ function $(\mathrm{U}, \mathrm{J}, \mathrm{s} 2, \mathrm{nMCMC})$

\{

\#log-posteriori density

te $=\operatorname{nrow}(\mathrm{U})$

gridTime $=(0:($ te -1$)) /$ te

$\log \operatorname{Pos}=$ function $(\operatorname{coefs})$

\{

valor $=0$

for $(\mathrm{k}$ in $1:$ te $)$

\{

alpha $=$ invPsi $($ haar $(\operatorname{coefs}, \operatorname{gridTime}[\mathrm{k}]))$

\# valor $=$ valor $+(\log D e n s C o p(a l p h a, U[k, 1], U[k, 2]))$

valor $=$ valor $+\operatorname{dCopula}(\mathrm{c}(\mathrm{U}[\mathrm{k}, 1], \mathrm{U}[\mathrm{k}, 2]), \operatorname{normalCopula}($ alpha $), \log =\mathrm{T})$

\}

ce $=\operatorname{matrix}\left(\right.$ coefs, nrow $=2^{\prec} \mathrm{J}$, ncol $\left.=1\right)$

\# valor $=$ valor $+\log$ Normal $(r e p(0, N)$, mcov, coefs $)$

valor $=$ valor $-\mathrm{t}($ ce $) \% * \%$ ce $/(2 * \mathrm{~s} 2)$

return (valor)

\}

\section{\#MCMC simulation}

dist $=$ make. $\operatorname{dist}\left(2^{\curlywedge} \mathrm{J}, \quad\right.$ 'post', $\left.\log \cdot \operatorname{density}=\log \mathrm{Pos}\right)$

$\# \mathrm{x} 0=\operatorname{runif}(\mathrm{N},-1,1)$

$\mathrm{x} 0=\operatorname{rep}\left(0,2^{\curlywedge} \mathrm{J}\right)$

amostra $=$ adaptive. metropolis. $\operatorname{sample}($ dist, $\mathrm{x} 0$, nMCMC, burn. $\mathrm{in}=1)$

estCoef $=$ colMeans (amostra $\$$ X) \#mean of chain generate by MCMC method

return (estCoef)

\} 


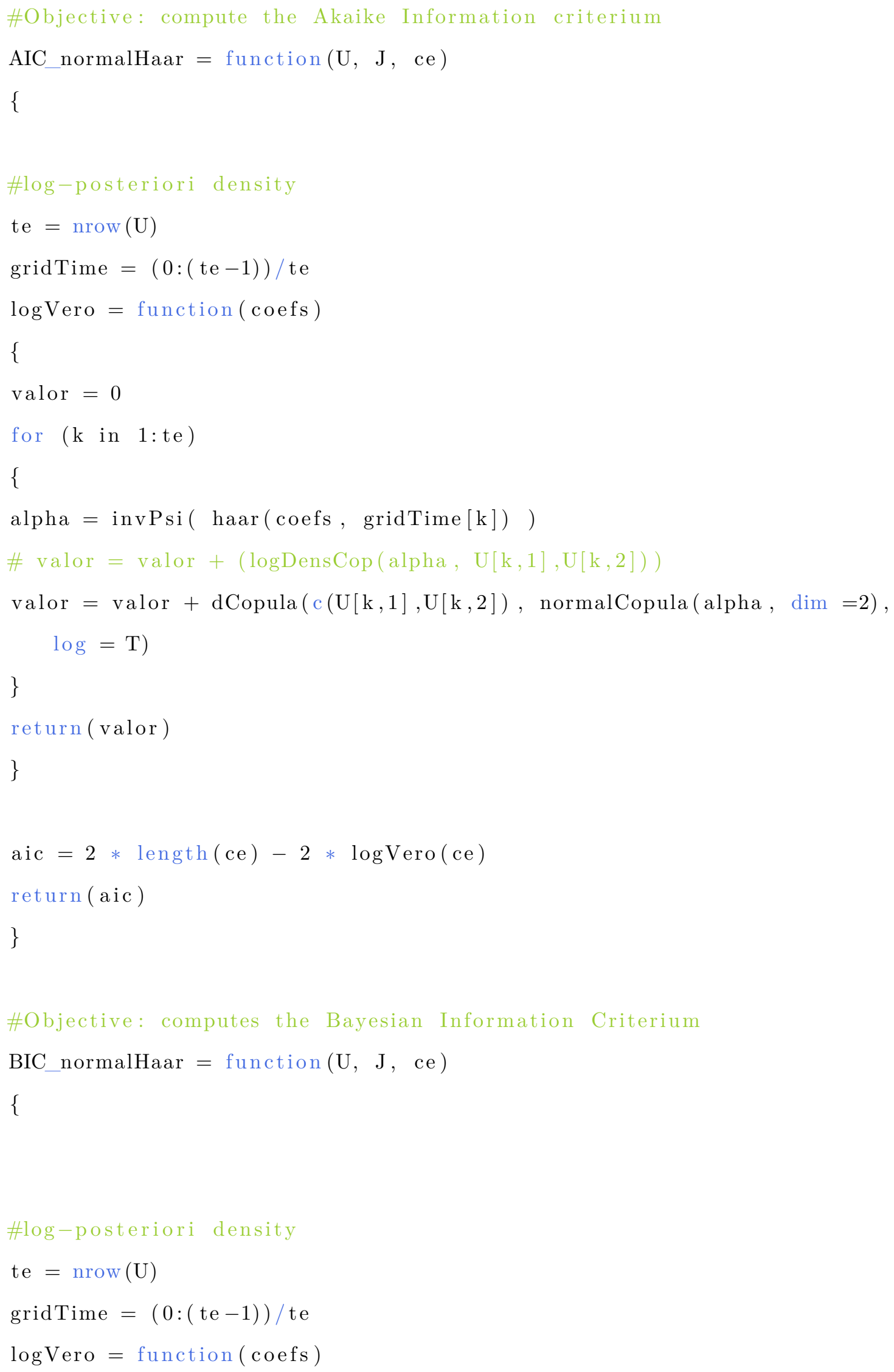




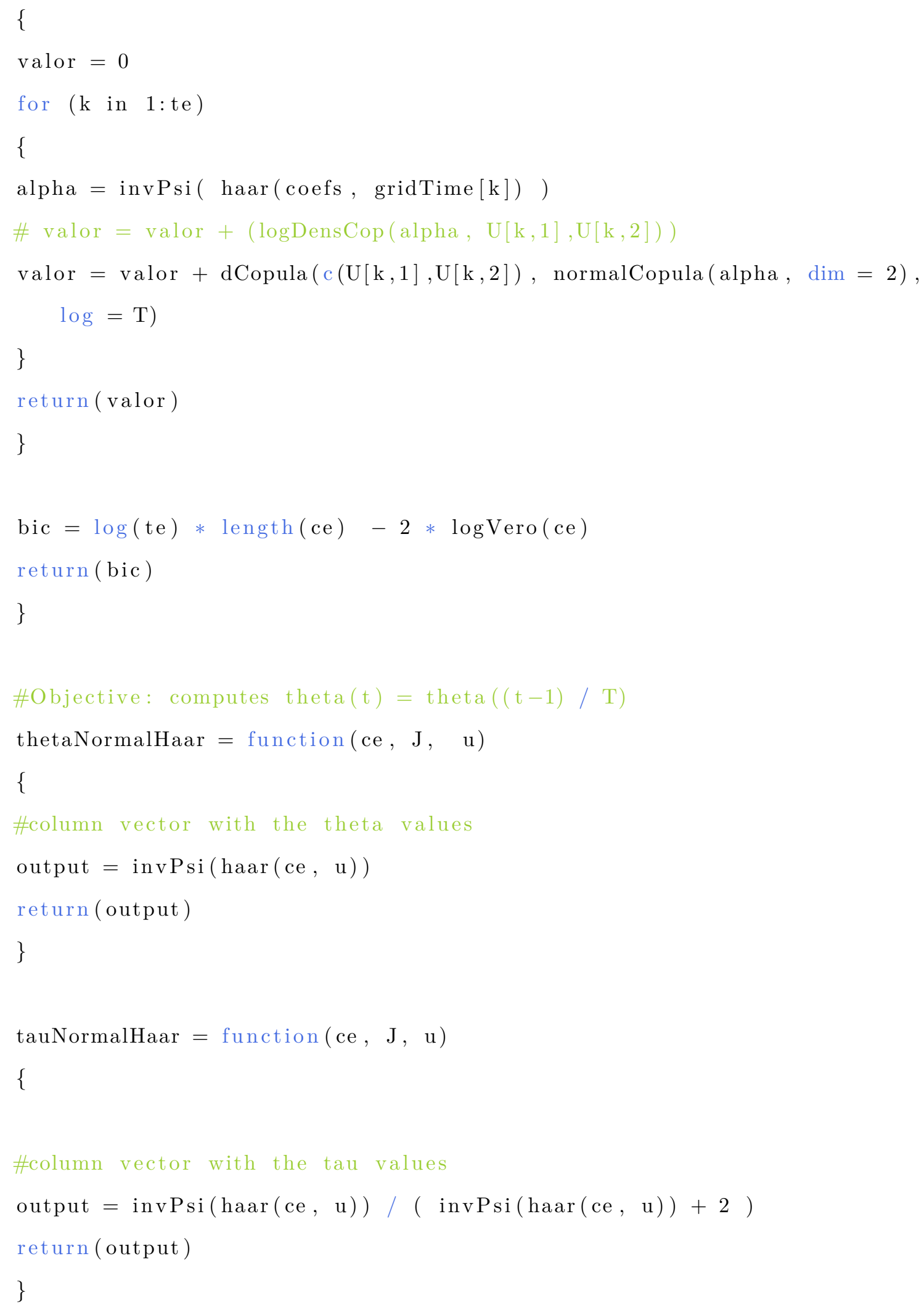




\section{A.2.3 Taylor}

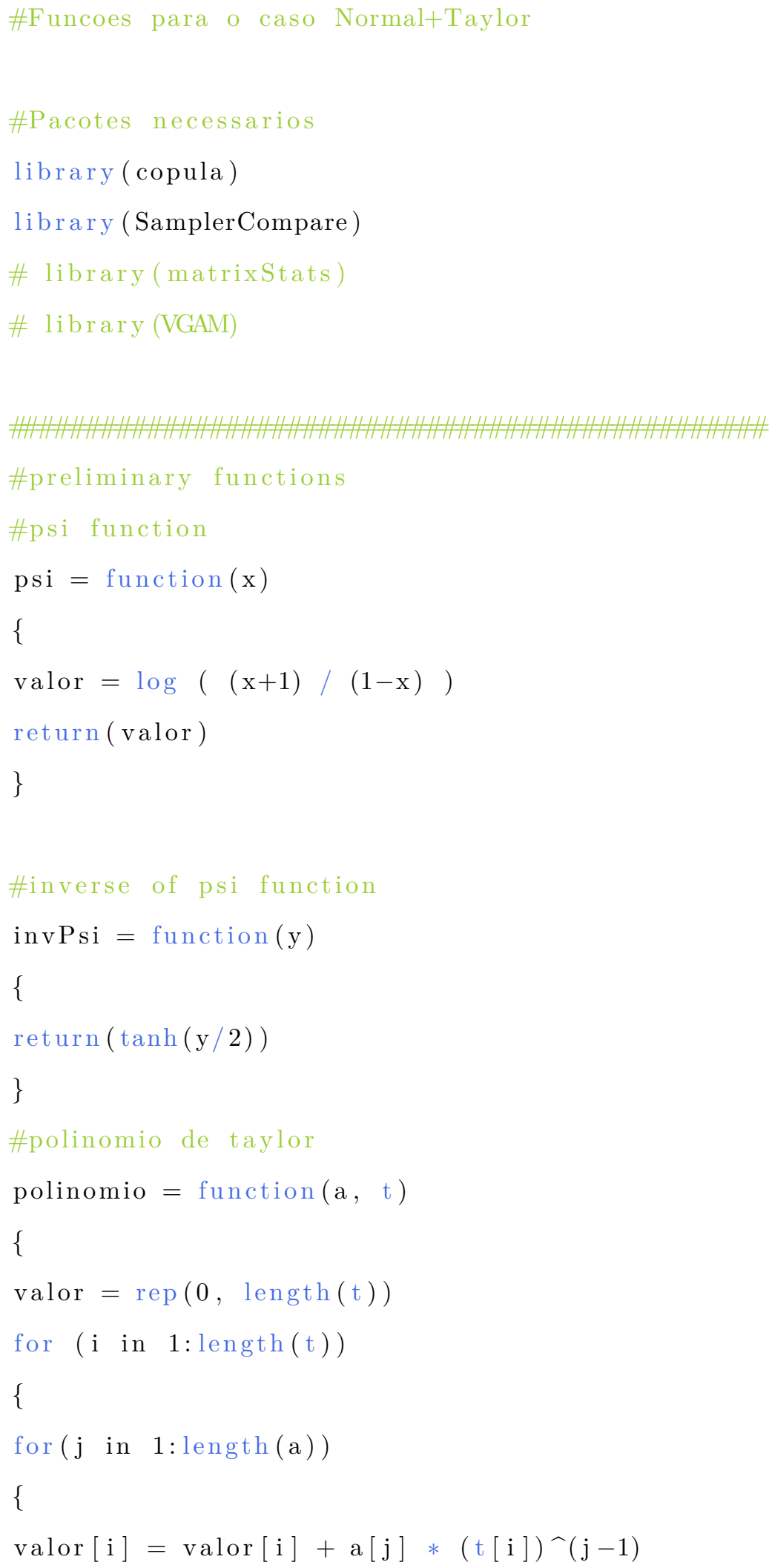




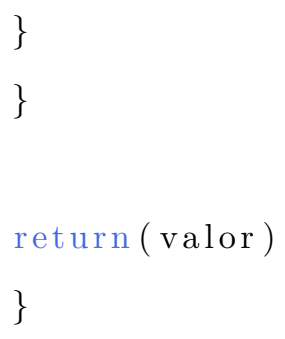




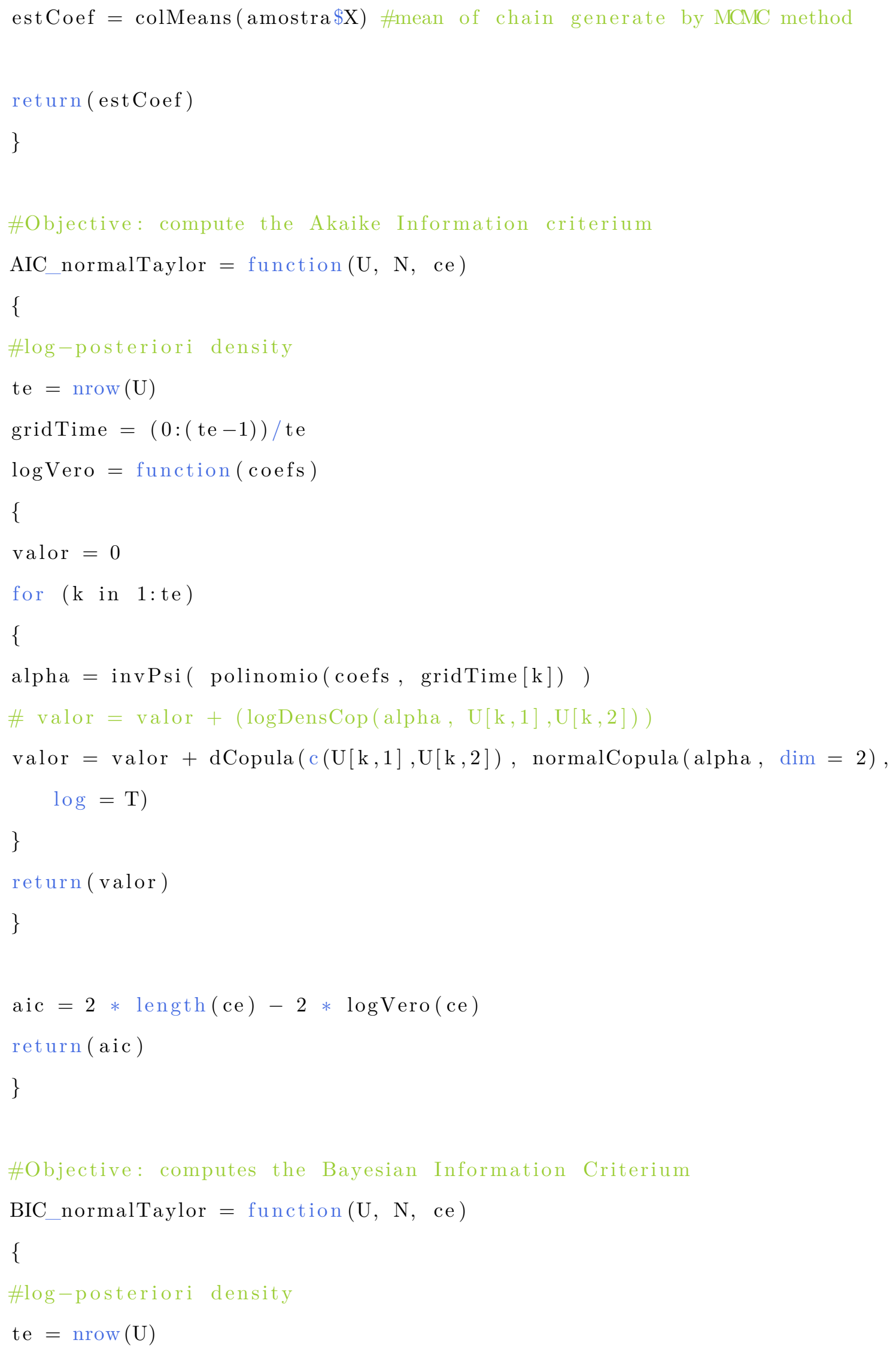




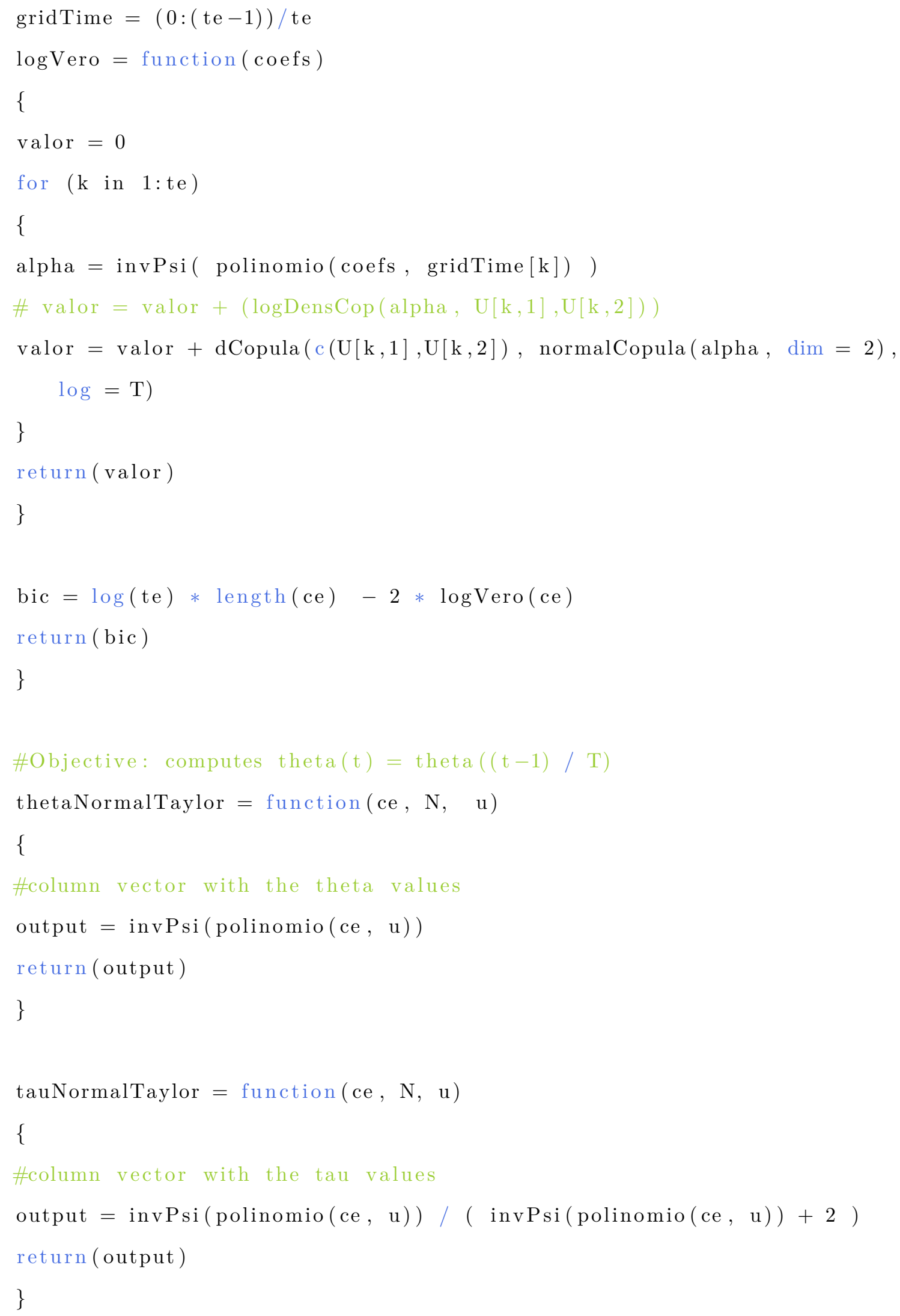




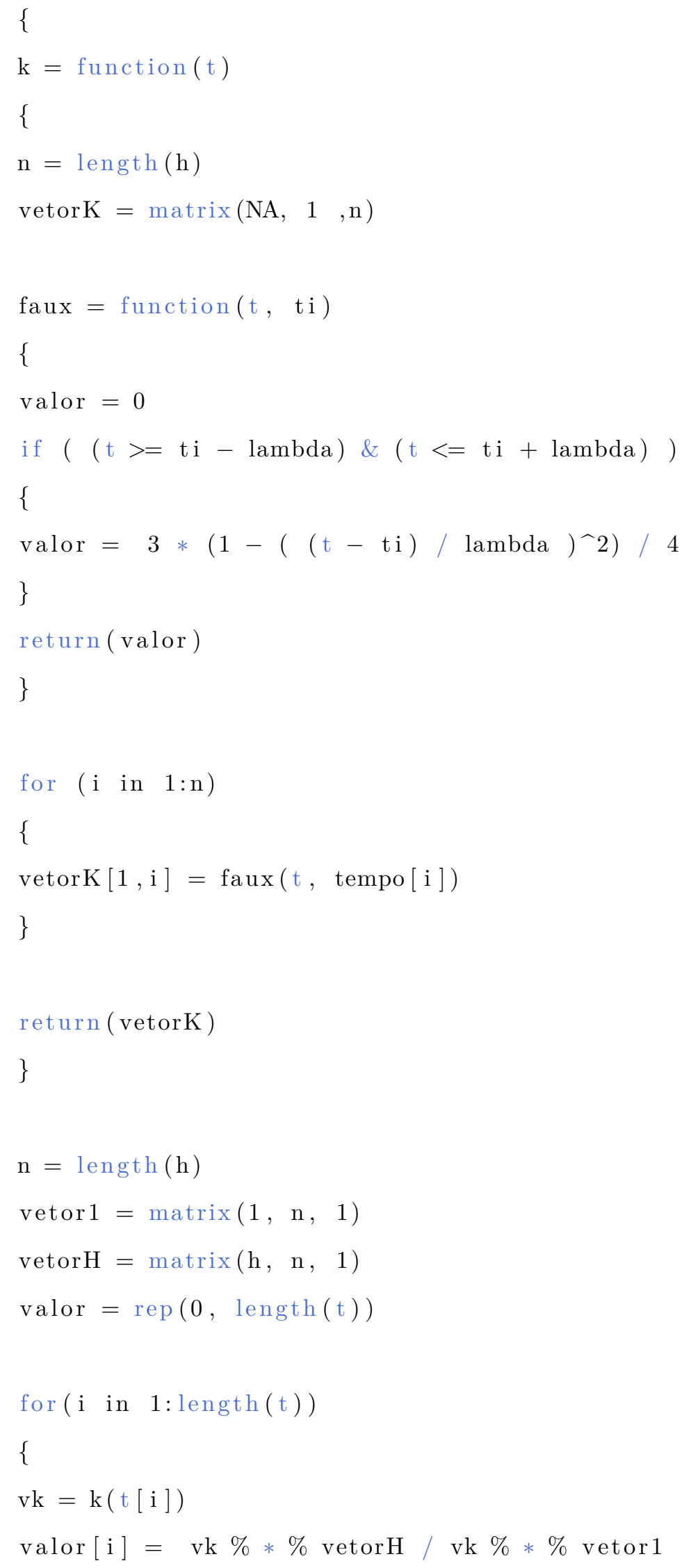




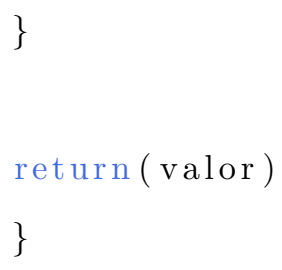




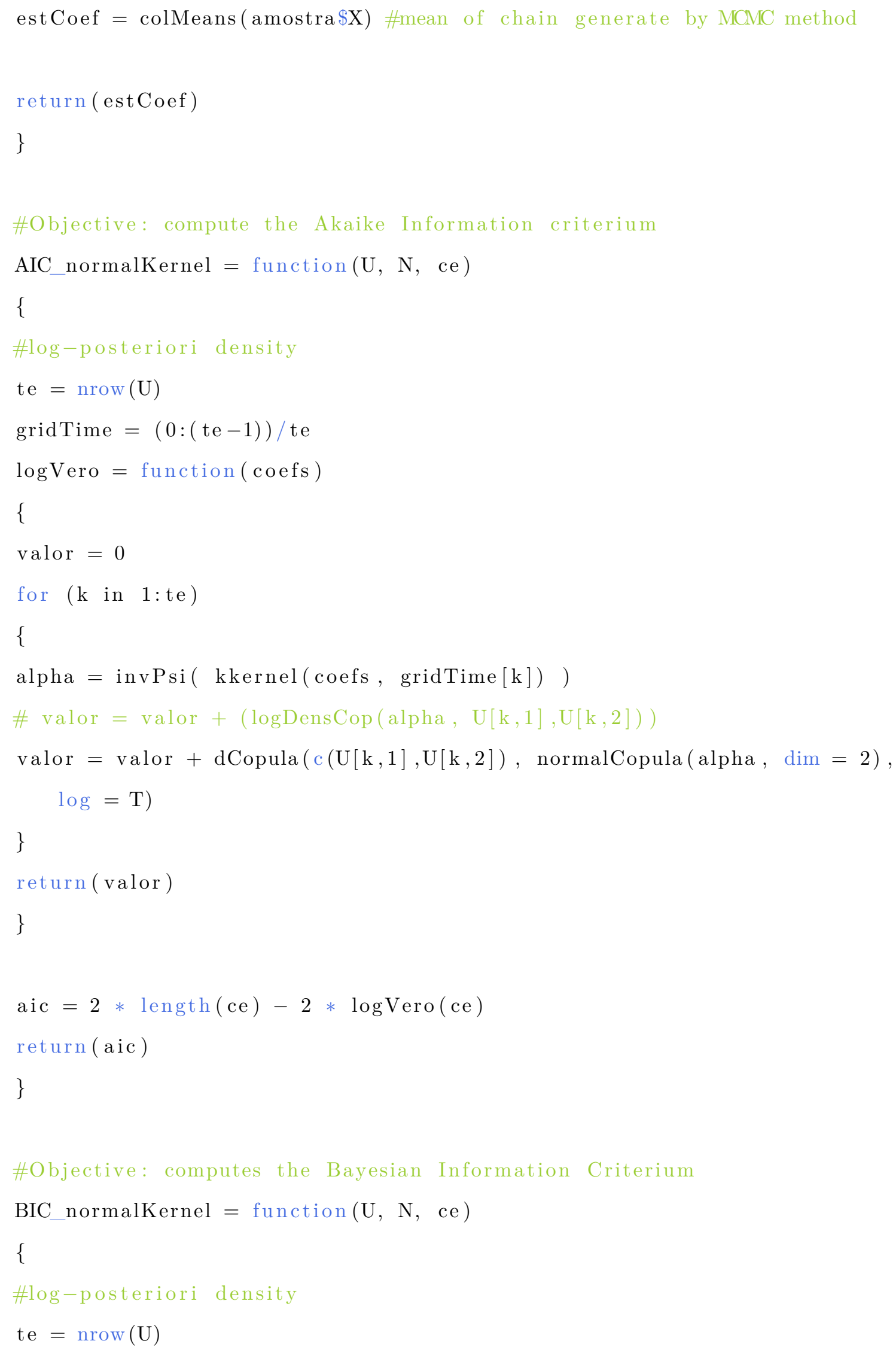




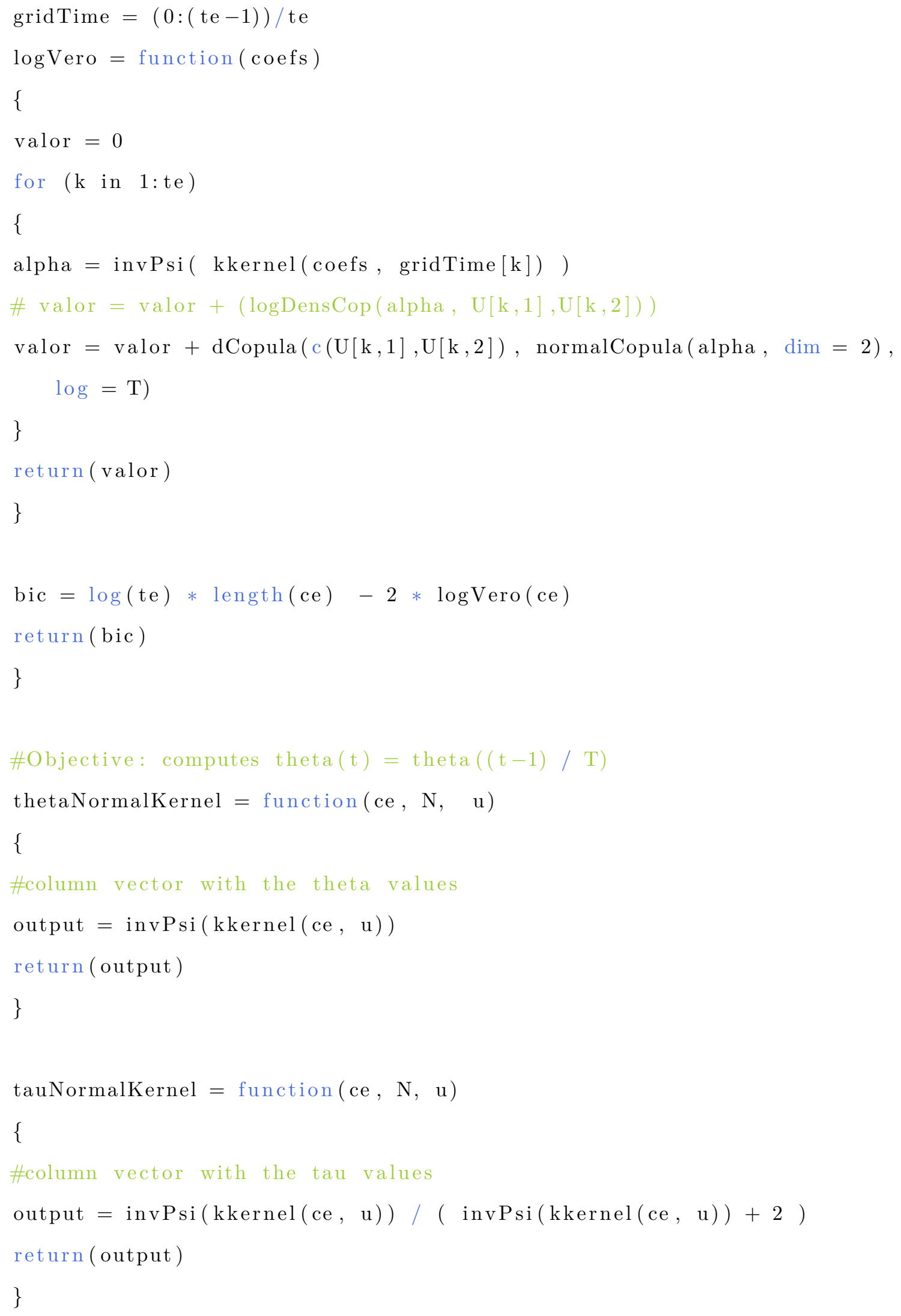




\title{
Referências Bibliográficas
}

\author{
Abegaz et al.(2012) Fentaw Abegaz, Irène Gijbels e Noël Veraverbeke. Semiparametric \\ estimation of conditional copulas. Journal of Multivariate Analysis, 110:43-73. Citado na \\ pág.
}

Almeida e Czado(2012) Carlos Almeida e Claudia Czado. Efficient bayesian inference for stochastic time-varying copula models. Computational Statistics \& Data Analysis, 56 (6):1511-1527. Citado na pág.

Aloui et al.(2013) Riadh Aloui, Shawkat Hammoudeh e Duc Khuong Nguyen. A timevarying copula approach to oil and stock market dependence: The case of transition economies. Energy Economics, 39:208-221. Citado na pág.

Atkinson et al.(2010) Richard W Atkinson, Gary W Fuller, H Ross Anderson, Roy M Harrison e Ben Armstrong. Urban ambient particle metrics and health: a time-series analysis. Epidemiology, 21(4):501-511. Citado na pág.

Ausin e Lopes(2010) M Concepcion Ausin e Hedibert F Lopes. Time-varying joint distribution through copulas. Computational Statistics \& Data Analysis, 54(11):2383-2399. Citado na pág.

Bartle e Sherbert(1992) Robert Gardner Bartle e Donald R Sherbert. Introduction to real analysis, volume 2. Wiley New York. Citado na pág. 
Boggess e Narcowich(2009) Albert Boggess e Francis J Narcowich. A first course in wavelets with Fourier analysis. John Wiley \& Sons. Citado na pág.

Bollerslev(1986) Tim Bollerslev. Generalized autoregressive conditional heteroskedasticity. Journal of econometrics, 31(3):307-327. Citado na pág.

Bouyé et al.(2000) Eric Bouyé, Valdo Durrleman, Ashkan Nikeghbali, Gaël Riboulet e Thierry Roncalli. Copulas for finance-a reading guide and some applications. Available at SSRN 1032533. Citado na pág.

Brooks et al.(2011) Steve Brooks, Andrew Gelman, Galin L Jones e Xiao-Li Meng. Handbook of Markov Chain Monte Carlo. Chapman and Hall/CRC. Citado na pág.

Cherubini e Luciano(2001) Umberto Cherubini e Elisa Luciano. Value-at-risk trade-off and capital allocation with copulas. Economic notes, 30(2):235-256. Citado na pág.

Christoffersen et al.(2010) Peter Christoffersen, Vihang R Errunza, Kris Jacobs e Xisong Jin. Is the potential for international diversification disappearing? Available at SSRN 1573345. Citado na pág.

Chui(2014) Charles K Chui. An introduction to wavelets, volume 1. Academic press. Citado na pág.

Creal et al.(2013) Drew Creal, Siem Jan Koopman e André Lucas. Generalized autoregressive score models with applications. Journal of Applied Econometrics, 28(5):777-795. Citado na pág.

Daubechies et al.(1992) Ingrid Daubechies et al. Ten lectures on wavelets, volume 61. SIAM. Citado na pág.

Deb et al.(2014) Partha Deb, Pravin K Trivedi e David M Zimmer. Cost-offsets of prescription drug expenditures: Data analysis via a copula-based bivariate dynamic hurdle model. Health economics, 23(10):1242-1259. Citado na pág. 
Denuit et al.(2006) Michel Denuit, Jan Dhaene, Marc Goovaerts e Rob Kaas. Actuarial theory for dependent risks: measures, orders and models. John Wiley \& Sons. Citado na pág.

Embrechts et al.(2001) Paul Embrechts, Filip Lindskog e Alexander McNeil. Modelling dependence with copulas. Rapport technique, Département de mathématiques, Institut Fédéral de Technologie de Zurich, Zurich. Citado na pág.

Embrechts et al.(2013) Paul Embrechts, Claudia Klüppelberg e Thomas Mikosch. Modelling extremal events: for insurance and finance, volume 33. Springer Science \& Business Media. Citado na pág.

Engle(1982) Robert F Engle. Autoregressive conditional heteroscedasticity with estimates of the variance of united kingdom inflation. Econometrica: Journal of the Econometric Society, páginas 987-1007. Citado na pág.

Fermanian e Wegkamp(2004) Jean-David Fermanian e MARTEN Wegkamp. Time dependent copulas. Preprint INSEE, Paris, France. Citado na pág.

Friedman et al.(2001) Jerome Friedman, Trevor Hastie e Robert Tibshirani. The elements of statistical learning, volume 1. Springer series in statistics Springer, Berlin. Citado na pág.

Haar(1909) Kármán Th. v. Haar, A. Zur theorie der spannungszustände in plastischen und sandartigen medien. Nachrichten von der Gesellschaft der Wissenschaften zu Göttingen, Mathematisch-Physikalische Klasse, 1909:204-218. Citado na pág.

Haario et al.(2001) Heikki Haario, Eero Saksman e Johanna Tamminen. An adaptive metropolis algorithm. Bernoulli, páginas 223-242. Citado na pág.

Härdle et al.(1998) Wolfgang Härdle, Gerard Kerkyacharian, Alexander Tsybakov e Dominique Picard. Wavelets, approximation, and statistical applications. Springer. Citado na pág. 
Harrison(1996) Ann Harrison. Openness and growth: A time-series, cross-country analysis for developing countries. Journal of development Economics, 48(2):419-447. Citado na pág.

Hastings(1970) W Keith Hastings. Monte carlo sampling methods using markov chains and their applications. Biometrika, 57(1):97-109. Citado na pág.

Horová et al.(2012) Ivanka Horová, Jan Koláček e Jiř́i Zelinka. Kernel Smoothing in MATLAB: theory and practice of kernel smoothing. World scientific. Citado na pág.

Hutchinson e Lai(1990) Timothy P Hutchinson e Chin Diew Lai. Continuous bivariate distributions, emphasising applications. Rumsby Scientific Publishing Adelaide. Citado na pág.

Joe(1997) Harry Joe. Multivariate models and multivariate dependence concepts. CRC Press. Citado na pág.

Jondeau e Rockinger(2006) Eric Jondeau e Michael Rockinger. The copula-garch model of conditional dependencies: An international stock market application. Journal of international money and finance, 25(5):827-853. Citado na pág.

Kimeldori e Sampson(1975) George Kimeldori e Allan Sampson. Uniform representations of bivariate distributions. Communications in Statistics-Theory and Methods, 4(7): 617-627. Citado na pág.

Klugman et al.(2012) Stuart A Klugman, Harry H Panjer e Gordon E Willmot. Loss models: from data to decisions, volume 715. John Wiley \& Sons. Citado na pág.

Kojadinovic e Yan(2010) Ivan Kojadinovic e Jun Yan. Modeling multivariate distributions with continuous margins using the copula R package. Journal of Statistical Software, 34(9):1-20. URL http://www.jstatsoft.org/v34/i09/. Citado na pág. 
Kovats et al.(2004) RS Kovats, SJ Edwards, S Hajat, BG Armstrong, KL Ebi e B Menne. The effect of temperature on food poisoning: a time-series analysis of salmonellosis in ten european countries. Epidemiology and Infection, 132(03):443-453. Citado na pág.

Manner e Reznikova(2012) Hans Manner e Olga Reznikova. A survey on time-varying copulas: specification, simulations, and application. Econometric Reviews, 31(6):654-687. Citado na pág.

Metropolis et al.(1953) Nicholas Metropolis, Arianna W Rosenbluth, Marshall N Rosenbluth, Augusta H Teller e Edward Teller. Equation of state calculations by fast computing machines. The journal of chemical physics, 21(6):1087-1092. Citado na pág.

Meyer e Ryan(1993) Y Meyer e RD Ryan. Wavelets: Algorithms and applications. SIAM, Philadelphia, PA. Citado na pág.

Meyer(1985) Yves Meyer. Principe d'incertitude, bases hilbertiennes et algebres d'operateurs. Séminaire Bourbaki, 28:209-223. Citado na pág.

Meyer e Salinger(1995) Yves Meyer e David H Salinger. Wavelets and operators, volume 1. Cambridge University Press. Citado na pág.

Morettin(2014) Pedro A Morettin. Ondas e Ondaletas: Da Análise de Fourier à Análise de Ondaletas de Séries Temporais. São Paulo: Editora USP. Citado na pág.

Morettin e Toloi(2006) Pedro A Morettin e Clélia Toloi. Análise de séries temporais. Blucher. Citado na pág.

Nelsen(2007) Roger B Nelsen. An introduction to copulas. Springer Science \& Business Media. Citado na pág.

Nelsen(2013) Roger B Nelsen. An introduction to copulas, volume 139. Springer Science \& Business Media. Citado na pág. 
Patton(2004) Andrew J Patton. On the out-of-sample importance of skewness and asymmetric dependence for asset allocation. Journal of Financial Econometrics, 2(1):130-168. Citado na pág.

Patton(2006) Andrew J Patton. Estimation of multivariate models for time series of possibly different lengths. Journal of applied econometrics, 21(2):147-173. Citado na pág.

Patton(2012) Andrew J Patton. A review of copula models for economic time series. Journal of Multivariate Analysis, 110:4-18. Citado na pág.

Patton(2002) Andrew John Patton. Applications of copula theory in financial econometrics. Tese de Doutorado, University of California, San Diego. Citado na pág.

Petit et al.(1999) Jean-Robert Petit, Jean Jouzel, Dominique Raynaud, Narcisse I Barkov, J-M Barnola, Isabelle Basile, Michael Bender, J Chappellaz, M Davis, G Delaygue et al. Climate and atmospheric history of the past 420,000 years from the vostok ice core, antarctica. Nature, 399(6735):429-436. Citado na pág.

Prescott et al.(1998) GJ Prescott, GR Cohen, RA Elton, FG Fowkes e RM Agius. Urban air pollution and cardiopulmonary ill health: a 14.5 year time series study. Occupational and environmental medicine, 55(10):697-704. Citado na pág.

R Core Team(2015) R Core Team. R: A Language and Environment for Statistical Computing. R Foundation for Statistical Computing, Vienna, Austria, 2015. URL https: //www.R-project.org/. Citado na pág.

Renard e Lang(2007) Benjamin Renard e Michel Lang. Use of a gaussian copula for multivariate extreme value analysis: some case studies in hydrology. Advances in Water Resources, 30(4):897-912. Citado na pág.

Roberts e Rosenthal(2009) Gareth O Roberts e Jeffrey S Rosenthal. Examples of adaptive mcmc. Journal of Computational and Graphical Statistics, 18(2):349-367. Citado na pág. 
Roberts et al.(2001) Gareth O Roberts, Jeffrey S Rosenthal et al. Optimal scaling for various metropolis-hastings algorithms. Statistical science, 16(4):351-367. Citado na pág.

Salvadori e De Michele(2007) G Salvadori e C De Michele. On the use of copulas in hydrology: theory and practice. Journal of Hydrologic Engineering, 12(4):369-380. Citado na pág.

Scaillet e Fermanian(2002) Olivier Scaillet e Jean-David Fermanian. Nonparametric estimation of copulas for time series. FAME Research paper, (57). Citado na pág.

Shiau et al.(2007) Jenq-Tzong Shiau, Song Feng e Saralees Nadarajah. Assessment of hydrological droughts for the yellow river, china, using copulas. Hydrological Processes, 21(16):2157-2163. Citado na pág.

Shumway e Stoffer(2013) Robert H Shumway e David S Stoffer. Time series analysis and its applications. Springer Science \& Business Media. Citado na pág.

Silva Filho et al.(2014) Osvaldo C Silva Filho, Flavio A Ziegelmann e Michael J Dueker. Assessing dependence between financial market indexes using conditional time-varying copulas: applications to value at risk (var). Quantitative Finance, 14(12):2155-2170. Citado na pág.

Sklar(1973) Abe Sklar. Random variables, joint distribution functions, and copulas. Kybernetika, 9(6):449-460. Citado na pág.

Sklar(1959) M Sklar. Fonctions de répartition à $n$ dimensions et leurs marges. Université Paris 8. Citado na pág.

Styan(1973) George PH Styan. Hadamard products and multivariate statistical analysis, volume 6. Elsevier. Citado na pág.

Thompson(2011) Madeleine B. Thompson. Introduction to SamplerCompare. Journal of Statistical Software, 43(12):1-10. URL http://www.jstatsoft.org/v43/i12/. Citado na pág. 
Wand e Jones(1994) Matt P Wand e M Chris Jones. Kernel smoothing. Crc Press. Citado na pág.

Zeger et al.(2006) Scott L Zeger, Rafael Irizarry e Roger D Peng. On time series analysis of public health and biomedical data. Annual Review of Public Health, 27:57-79. Citado na pág.

Zhang e Guegan(2008) Jing Zhang e Dominique Guegan. Pricing bivariate option under garch processes with time-varying copula. Insurance: Mathematics and Economics, 42(3): 1095-1103. Citado na pág.

Zhao e Zhou(2012) Xiaobing Zhao e Xian Zhou. Estimation of medical costs by copula models with dynamic change of health status. Insurance: Mathematics and Economics, 51(2):480-491. Citado na pág.

Zivot e Wang(2007) Eric Zivot e Jiahui Wang. Modeling financial time series with SPlusß, volume 191. Springer Science \& Business Media. Citado na pág. 